Draft Version September 3, 2018

Preprint typeset using $\mathrm{LATE}_{\mathrm{E} X}$ style emulateapj v. 12/16/11

\title{
HABITABILITY OF EXOPLANET WATERWORLDS
}

\author{
EDWin S. KITE \\ Department of Geophysical Sciences, University of Chicago, Chicago, IL 60637, USA \\ kite@uchicago.edu
}

ERIC B. FORD

Department of Astronomy and Astrophysics, The Pennsylvania State University, University Park, PA 16802, USA

Center for Exoplanets and Habitable Worlds, The Pennsylvania State University, University Park, PA 16802, USA Institute for CyberScience, The Pennsylvania State University, University Park, PA 16802, USA and

Pennsylvania State Astrobiology Research Center, The Pennsylvania State University, University Park, PA 16802, USA

Draft version September 3, 2018

\begin{abstract}
Many habitable zone (HZ) exoplanets are expected to form with water mass fractions higher than that of the Earth. For rocky exoplanets with 10-1000× Earth's $\mathrm{H}_{2} \mathrm{O}$ but without $\mathrm{H}_{2}$, we model the multiGyr evolution of ocean temperature and chemistry, taking into account $\mathrm{C}$ partitioning, high-pressure ice phases, and atmosphere-lithosphere exchange. Within our model, for Sun-like stars, we find that: (1) the duration of habitable surface water is strongly affected by ocean chemistry; (2) possible ocean $\mathrm{pH}$ spans a wide range; (3) surprisingly, many waterworlds retain habitable surface water for $>1$ Gyr, and (contrary to previous claims) this longevity does not necessarily involve geochemical cycling. The key to this cycle-independent planetary habitability is that $\mathrm{C}$ exchange between the convecting mantle and the water ocean is curtailed by seafloor pressure on waterworlds, so the planet is stuck with the ocean mass and ocean cations that it acquires during the first $1 \%$ of its history. In our model, the sum of positive charges leached from the planetary crust by early water-rock interactions is - coincidentally - often within an order of magnitude of the early-acquired atmosphere+ocean inorganic C inventory overlaps. As a result, $p_{\mathrm{CO} 2}$ is frequently in the "sweet spot" (0.2-20 bar) for which the range of semimajor axis that permits surface liquid water is about as wide as it can be. Because the width of the HZ in semimajor axis defines (for Sun-like stars) the maximum possible time span of surface habitability, this effect allows for Gyr of habitability as the star brightens. We illustrate our findings by using the output of an ensemble of $N$-body simulations as input to our waterworld evolution code. Thus (for the first time in an end-to-end calculation) we show that chance variation of initial conditions, with no need for geochemical cycling, can yield multi-Gyr surface habitability on waterworlds.

Keywords: planets and satellites: individual (Kepler-452b, Kepler-1638b, Kepler-1606b, Kepler-1090b, Kepler-22b, $\tau$ Ceti e, Proxima Cen b, TRAPPIST-1, GJ 667 C, LHS 1140 b, Ross 128 b, Kepler-62f, Kepler-186f, GJ 832 c, HD 40307 g, Kepler-442b, Kepler-1229 b)
\end{abstract}

\section{INTRODUCTION.}

Habitable zone (HZ) small-radius exoplanets are common (Burke et al. 2015; Dressing \& Charbonneau 2015). What fraction of them are habitable? For the purposes of this paper, a useful definition of a potentially habitable exoplanet is that it maintains $T<450 \mathrm{~K}$ liquid water on its surface continuously for timescales that are relevant for biological macroevolution, $\gg 10^{7}$ yr (Vermeij 2006, Carter 1983, Bains et al. 2015) 1 . With this definition, two pathways allow long-term planetary habitability:

Habitability sustained by geochemical cycles: Planets that stay habitable, due to a negative feedback, when climate is perturbed (by tectonics, stellar evolution, etc.). A proposed mechanism for the negative feedback is a geochemical cycle that balances volcanic outgassing and the fixation of atmospheric gases into rocks (carbonate-silicate weathering feedback; e.g. Walker et al. 1981, Kasting et al. 1993).

\footnotetext{
${ }^{1}$ Sub-ice oceans in extrasolar planetary systems may be habitable, but this cannot be confirmed from Earth by remote sensing.
}

Climate-stabilizing geochemical cycles are a mainstay of textbooks and review papers (Catling \& Kasting 2017; Knoll et al. 2012; Kaltenegger 2017, and references therein). However, such feedbacks probably do not work on HZ rocky planets with water mass fractions $10 \times$ $10^{3} \times$ that of the Earth - "waterworlds" (Foley 2015 Abbot et al. 2012). Yet waterworlds should be common in the Galaxy (e.g. Mulders et al. 2015). Are waterworlds doomed? Not necessarily, because there is another (lessstudied) road to long-term planetary habitability.

Cycle-independent planetary habitability: Planets where $p_{\mathrm{CO} 2}$, ocean depth, and surface temperature $T_{\text {surf }}$ remain within the habitable range for $\gg 10^{7}$ yr without geochemical cycling.

In this paper, we first show that the long-term climate evolution of waterworlds can be modeled independently of long-term geochemical cycling between the atmosphere and the convecting silicate-rock mantle (Section 2). Next, we set up (Section 3) and run (Section 4) such a waterworld evolution model. Using the model, we estimate what combinations of water abundance, initial 


\section{CYCLE-DEPENDENT} PLANETARY HABITABILITY fast atmosphere-interior cycling: atmosphere+ocean C content adjusted by negative feedbacks

surface water $=1 \times$ Earth

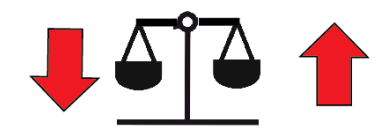

$\tau_{\mathrm{CO},(\mathrm{A} / \mathrm{O})-\mathrm{I}} \sim 10^{5} \mathrm{yr}$ interior

surface water $<10 \times$ Earth not considered in this paper

\section{WATERWORLDS: CYCLE-INDEPENDENT} PLANETARY HABITABILITY sluggish atmosphere-interior cycling: atmosphere+ocean C content conserved after $10^{8} \mathrm{yr}$

$\tau_{\mathrm{CO} 2,(\mathrm{~A} / \mathrm{O})-\mathrm{I}}>10^{10} \mathrm{yr}$

Figure 1. Cycle-independent planetary habitability on exoplanet waterworlds. On shallow-ocean planets such as the Earth (top row), the timescale for (atmosphere/ocean) $\leftrightarrow$ interior exchange of $\mathrm{CO}_{2}\left(\tau_{\mathrm{CO} 2},(\mathrm{~A} / \mathrm{O}) \leftrightarrow \mathrm{I}\right)$ can be much shorter than the host star's main-sequence lifetime. With such fast cycling, small mismatches in the $\mathrm{C}$ cycle can quickly build up to a big change in greenhouse forcing, and big change in surface temperature - a threat to surface habitability (Figure 21). Therefore, geochemical cycles are thought to be central to sustained surface habitability on shallow-ocean planets. By contrast, on deep-water planets (bottom row), atmosphere-interior cycling is curtailed by high seafloor pressure; $\tau_{\mathrm{CO} 2,(\mathrm{~A} / \mathrm{O}) \leftrightarrow \mathrm{I}}$ can be $>10$ Gyr. Under such circumstances, long-term geochemical cycling between the atmosphere and the convecting silicate-rock mantle does not matter for determining whether or not the planet has habitable surface water for Gyr. Instead, the duration of habitable surface water is set by (i) atmosphere-ocean partitioning of $\mathrm{C}$, and (ii) the initial cation content of the water ocean as it emerges from the first $100 \mathrm{Myr}$ of giant impacts, late-stage delivery of $\mathrm{C}$, and early crust formation.

carbon abundance, and geologic processes allow habitable surface water to persist for $>1$ Gyr. We focus on $\mathrm{CO}_{2}+\mathrm{H}_{2} \mathrm{O}\left( \pm \mathrm{N}_{2}\right)$ atmospheres (e.g. Wordsworth \& Pierrehumbert 2013a), for which the key climate-regulating greenhouse gas is $\mathrm{CO}_{2}$. Our central result is that the partial pressure of atmospheric $\mathrm{CO}_{2}\left(p_{\mathrm{CO} 2}\right)$ in our waterworld model is frequently in the $\sim 0.2-20$ bar range that enables $>1$ Gyr of surface liquid water. We ignore $\mathrm{H}_{2}$ warming (Stevenson 1999). We emphasize long-term climate evolution for planets around Sun-like stars, and defer discussion of nutrients to Section 6. In Section 5, we use an $N$-body model of planet assembly to demonstrate how waterworlds form and migrate to the HZ. Readers interested only in astrophysics may skip Sections 3 and 4; readers interested only in geoscience may skip Section 5. We conclude in Section 7. Our main results are shown in Figures 1517

\subsection{This paper in context}

Simulations of rocky-planet formation yield many rocky small-radius $\left(R_{p l}<1.6 \quad R_{\oplus}\right)$ planets that have planet water mass fractions $f_{W}=10-1000 \times f_{W, \oplus}$ (Raymond et al. 2004, 2007; Zain et al. 2018; Ciesla et al. 2015. Mulders et al. 2015). Earth's $f_{W}\left(\sim 10^{-4}\right)$ appears to be the result of chance (e.g., Raymond et al. 2007, Schönbächler et al. 2010, Morbidelli et al. 2016. Lichtenberg et al. 2016) (Section 5.2). Indeed, simulations suggest $f_{W} \approx f_{W, \oplus}$ may be uncommon on planets

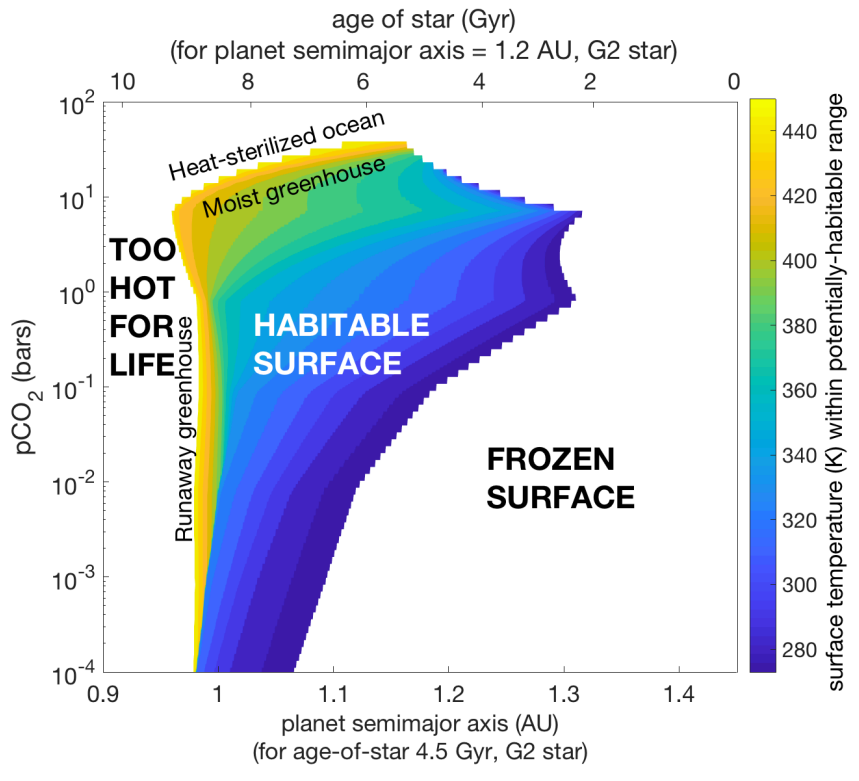

Figure 2. To show the effect of partial pressure of atmospheric $\mathrm{CO}_{2}$ and of insolation $L_{*}$ on habitability. For G-type stars, insolation increases with age of the star on the main sequence (top axis) and decreases with distance from the star (bottom axis). The colored region corresponds to the habitable zone (HZ). The width of the $\mathrm{HZ}$ in semimajor axis defines the maximum possible time span of surface habitability. The planet fates define a lozenge of habitability that is broadest for $0.2-20$ bars $p_{\mathrm{CO} 2}$. In other words, for a given semimajor axis, the duration of surface habitability is maximized for a "sweet spot" of $p_{\mathrm{CO} 2}$ in the range $0.2-20$ bar. The color ramp corresponds to $T_{\text {surf }}$, and extends from 273 to $450 \mathrm{~K}$. Note that increasing $p_{\mathrm{CO} 2}$. does not, by itself, cause a runaway greenhouse (Kasting \& Ackerman 1986 Ramirez et al. 2014). This plot is based on Wordsworth \& Pierrehumbert (2013a). (See Figure 19 below for a sensitivity test based on Ramirez et al. (2014)).

in general (e.g. Tian \& Ida 2015).

Among previous studies of waterworlds (e.g., Kuchner 2003; Léger et al. 2004; Selsis et al. 2007; Fu et al. |2010. Abbot et al. 2012; Levi et al. 2017; Unterborn et al.|2018), the closest in intent to our own are those of Kitzmann et al. (2015) and Noack et al. (2016). Kitzmann et al. (2015) consider carbonate-system equilibria and find a "sweet-spot" in total planet C similar to our own, but they do not consider geological processes. Noack et al. (2016) emphasize the development of high-pressure $\mathrm{H}_{2} \mathrm{O}$ ice (HP-ice) layers that may isolate the liquid ocean from the nutrients supplied by silicate-rock leaching. Noack et al. (2016) also confirm the result of Kite et al. (2009) that deep oceans suppress $\mathrm{C}$ exchange between the convecting mantle and the water ocean. We conservatively do not count planets with HP-ice as examples of habitability (see Section 6.4 for a discussion). This can set a $T_{\text {surf-dependent }}$ upper limit on the depth of a habitable ocean (provided $T_{\text {surf }} \lesssim 375 \mathrm{~K}$; Figure 3), and we consider only $<8 \mathrm{GPa}$ oceans. We go beyond Noack et al. (2016) by considering the effect of $\mathrm{C}$ on climate, by using an $N$-body code to calculate how volatile delivery and giant impacts "load the dice" by regulating the fraction of planets that can have cycle-independent planetary habitability, and - most importantly - by tracking $T_{\text {surf }}$ and $p_{\mathrm{CO} 2}$ for $10 \mathrm{Ga}$ on the habitable worlds we model.

Most of the physical and chemical processes we discuss have been investigated previously in an Earth context. The novel aspect of our paper is that we apply these 


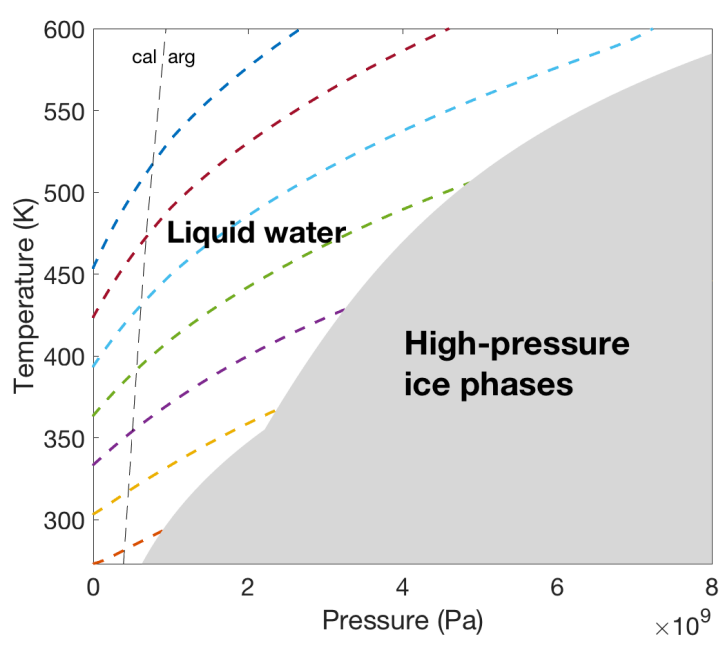

Figure 3. Maximum ocean pressure to avoid a frozen seafloor (high-pressure ice, HP-ice). The thick dashed lines correspond to liquid water adiabats (spaced at $30 \mathrm{~K}$ intervals in sea-surface temperature). For $T_{\text {surf }} \gtrsim 375 \mathrm{~K}$, liquid water becomes a supercritical fluid with increasing $P$, avoiding HP-ice. As the star brightens, sea-surface temperature increases, and it is possible for seafloor HP-ice to melt. The thin dashed line corresponds to the predominance boundary between the calcite (cal) and aragonite (arg) forms of $\mathrm{CaCO}_{3}$ (Redfern et al. 1989). For details, see Appendix B.

ideas to exoplanet waterworlds (Section 2).

\section{HOW TO MODEL HABITABILITY ON WATERWORLDS.}

Summary. Waterworlds are buffered against $\mathrm{H}_{2} \mathrm{O}$ loss (Section 2.1), but because $\mathrm{CO}_{2}$ modulates surface temperature and thus surface habitability (Section 2.2), we need to consider the chemistry that sets ocean $\mathrm{pH}$ and thus $\mathrm{CO}_{2}$ (Section 2.3). Fortunately, nature makes this task easier on waterworlds (Section 2.4), by chemically isolating the ocean+atmosphere from the convecting mantle after $10^{8} \mathrm{yr}$. This isolation is a necessary condition for cycle-independent planetary habitability.

\subsection{Waterworlds are buffered against $\mathrm{H}_{2} \mathrm{O}$ loss}

In a $\mathrm{CO}_{2}-\mathrm{H}_{2} \mathrm{O}$ atmosphere, high surface temperatures will cause high $\mathrm{H}_{2} \mathrm{O}$ mixing ratios in the stratosphere: this is referred to as a moist greenhouse state. In the moist greenhouse, $\mathrm{H}_{2} \mathrm{O}$ molecules are photolyzed by $\lambda \lesssim 240 \mathrm{~nm}$ photons, producing $\mathrm{H}_{2}$. This $\mathrm{H}_{2}$ is subsequently lost in a hydrodynamic outflow of the upper atmosphere, an outflow that is underpinned by absorption of $\lesssim 100 \mathrm{~nm}$ photons (Kasting 1988). Loss of $\mathrm{H}_{2}$ from $\mathrm{H}_{2} \mathrm{O}$ tends to dry out the planet, and so moistgreenhouse worlds are outside the "conservative HZ" limits of Kasting et al. (1993).

However, for an initial water endowment of $>50$ Earth oceans in the HZ of an FGK star, the moist greenhouse cannot cause complete ocean loss (Kasting 1988; Lammer et al. 2009, Luger \& Barnes 2015; Zahnle \& Catling 2017) (Figure 1). For example, integrating the XUV-flux estimate of Lammer et al. (2009) for $1 \mathrm{AU}$ and a G-type star gives a loss of no more than 20 Earth oceans over $4.5 \mathrm{Ga}$. This is small compared to the water available on waterworlds. Moreover, the XUV-limited water loss is an upper bound, as other processes likely limit the water loss to a lower value (e.g. Wordsworth \& Pierrehumbert 2013a: Kulikov et al. 2006; Tian 2015; Owen \& Alvarez 2016, Bourrier et al. 2017).
In principle, oceans can dry out if ocean water reacts with rocks to form hydrated minerals (Mustard 2018). In practice, for waterworlds that have solid rock interiors, atmosphere-interior exchange of $\mathrm{H}_{2} \mathrm{O}$ is not important for setting the surface water inventory. That is because solid Earth mantle rocks have an $\mathrm{H}$-storage capacity of only 1-10 Earth oceans (e.g. Hirschmann 2016); this is a small fraction of the total water available to a waterworld (Tikoo \& Elkins-Tanton 2017; for a contrary view, see Marty 2012). For such worlds, the ocean a planet has after cooling from the last giant impact is the ocean that planet will keep.

\subsection{Shallow-ocean worlds: vulnerable unless geochemical cycles maintain habitability.}

Planetary habitability is modulated by $\mathrm{CO}_{2}$ (Figure2). Very high partial pressures of atmospheric $\mathrm{CO}_{2}\left(p_{\mathrm{CO} 2}\right)$ lead to temperatures too high for life. However, intermediate $p_{\mathrm{CO} 2}$ can stave off global surface ice cover (up to a low-insolation limit where $\mathrm{CO}_{2}$ must condense) and thus extend habitability (Figure 2) (Kopparapu et al. 2013). $p_{\mathrm{CO} 2}$ would have varied widely if Earth had always lacked a negative feedback on $p_{\mathrm{CO} 2}$ (Kasting \& Catling 2003). On Earth, $>90 \%$ of $\mathrm{C}$ is stored in rocks, and $\mathrm{C}$ cycles between the atmosphere+ocean+biosphere and rocks every $\sim 300$ Kyr in the modern era (Knoll et al. 2012) - this is geologically rapid. As a result of this rapid geochemical cycling, a small initial imbalance between the rate of $\mathrm{C}$ release from rocks (volcanic/metamorphic outgassing) and $\mathrm{C}$ uptake into rocks (weathering uptake) could lead to global surface ice cover (a snowball) or alternatively lead to a moist greenhouse. An initial moist greenhouse with XUV-limited ocean loss would lead to loss of Earth's ocean water (Figures 1 and 21. Shallow-ocean rocky planets are vulnerable, unless geochemical cycles provide a negative feedback (e.g. carbonate-silicate weathering feedback) that can maintain habitability ${ }^{2}$

For worlds with oceans a few times deeper than the Earth's ocean - enough to drown the land, but not as deep as the oceans we model in this paper - it is more difficult to equalize $\mathrm{C}$ uptake into rocks and $\mathrm{C}$ outgassing (Foley 2015; Abbot et al. 2012). Even though C uptake by weathering of seafloor rocks might make up some of this imbalance (Coogan et al. 2016; Coogan and Gillis 2018), it is less likely that a small initial imbalance between $\mathrm{C}$ release and $\mathrm{C}$ uptake could be restored via a negative feedback on worlds with oceans a few times deeper than the Earth's.

\subsection{Calculating waterworld $p_{\mathrm{CO} 2}(t)$ : habitability is strongly affected by ocean chemistry}

Although waterworlds are buffered against water loss (Section 2.1), the surface temperature of that water icy, heat-sterilized, or somewhere in between? - depends on $p_{\mathrm{CO} 2}$ (Figure 2). $p_{\mathrm{CO} 2}$ on waterworlds is set by

2 Doom is not assured for a hypothetical shallow-ocean world without a negative feedback. Escape hatches include the following. (1) Neither the moist greenhouse transition nor the snowball transition is completely understood, and it is possible that they can self-arrest or reverse (Abbot et al. 2011 Abe et al. 2011 Abbot et al. 2012 Hu et al. 2011 Kodama et al. 2015 Hottman et al. 2017 Kodama et al. 2018). (2) The desirable $p_{\mathrm{CO} 2}$ for a planet near the inner edge of the $\mathrm{HZ}$ is small. For such a world, vigorous $\mathrm{C}$ uptake, feeble $\mathrm{C}$ outgassing, and an M-star host - if combined could allow $\gg 10$ Gyr of habitability. 
atmosphere-ocean partitioning of $\mathrm{C}$. The ocean is a major reservoir of $\mathrm{C}$ that equilibrates with the atmosphere on $<10^{8}$ yr timescales. For example, Earth's pre-industrial partition of $\mathrm{C}$ is 60 parts in the ocean for one part in air, with an atmosphere-ocean exchange time of $\sim 10^{3} \mathrm{yr}$.

Therefore, $p_{\mathrm{CO} 2}$ depends on $\mathrm{C}$ abundance, planet water mass fraction (三 dilution), and ocean $\mathrm{pH}$. pH matters because of the strong effect of $\mathrm{H}^{+}$activity on carbonate equilibria (Figure 4 ). $\mathrm{pH} \approx-\log _{10}\left[\mathrm{H}^{+}\right]$, where the square brackets here denote molarity. (Despite the similar notation, $\mathrm{pH}$ has nothing to do with the partial pressure of $\left.\mathrm{H}_{2}.\right) \quad p_{\mathrm{CO} 2}$ in the atmosphere is in equilibrium with dissolved $\mathrm{CO}_{2}$ in the ocean:

$$
p_{\mathrm{CO} 2}=\frac{\left[\mathrm{CO}_{2(\mathrm{aq})}\right]}{k_{H}}
$$

where the square brackets here denote concentration, and $k_{H}$ is a solubility constant. However, $p_{\mathrm{CO} 2}$ can be much less than expected by dividing the total inorganic $\mathrm{C}$ (i.e., $[\mathrm{C}]$ ) in the ocean by $k_{H}$. Specifically, if the $\mathrm{pH}$ is high enough to form $\mathrm{HCO}_{3}^{-}$or $\mathrm{CO}_{3}^{2-}$ at the expense of $\mathrm{CO}_{2(a q)}$ (Figure 4 ,

$$
\left(\mathrm{CO}_{2}(\mathrm{aq})+\mathrm{H}_{2} \mathrm{O} \rightleftharpoons \mathrm{H}_{2} \mathrm{CO}_{3}\right) \rightleftharpoons \mathrm{H}^{+}+\mathrm{HCO}_{3}^{2-} \rightleftharpoons 2 \mathrm{H}^{+}+\mathrm{CO}_{3}^{2-}
$$

then $p_{\mathrm{CO} 2}$ can be very low.

In this paper, we will refer to the sum of the electrically neutral species $\left(\mathrm{CO}_{2}(\mathrm{aq})\right.$ and $\left.\mathrm{H}_{2} \mathrm{CO}_{3}\right)$ as $\mathrm{CO}_{2}$; in Earth seawater, the concentration of $\left.\mathrm{H}_{2} \mathrm{CO}_{3}\right)$ is $\lesssim 0.3 \%$ that of $\mathrm{CO}_{2}(\mathrm{aq})$.

When ocean $\mathrm{pH}$ is high $\left(\mathrm{H}^{+}\right.$concentration is low $)$, then by Le Chatelier's principle carbonic acid gives up its $\mathrm{H}^{+}$. Thus, at high $\mathrm{pH}, \mathrm{C}$ is hosted in the ocean as $\mathrm{CO}_{3}^{2-}$ and $\mathrm{HCO}_{3}^{-}, \mathrm{CO}_{2(\mathrm{aq})}$ is low, and by Equation $1, p_{\mathrm{CO} 2}$ is low. In this case, the fraction of total $\mathrm{C}$ in the atmosphere is small (Figure 4). This describes the modern Earth Zeebe \& Wolf-Gladrow 2001; Ridgwell \& Zeebe 2005; Butler 1982, Zeebe 2012). In other words, high pH effectively sequesters $\mathrm{CO}_{2}$ from the atmosphere by driving the carbonate equilibria to the right. This raises the ratio $\left[\mathrm{CO}_{3}^{2-}\right] /\left[\mathrm{CO}_{2(\mathrm{aq})}\right]$ (Equation 2), and so decreases the $p_{\mathrm{CO} 2}$ in equilibrium with the ocean (Equation 1). For a fixed total atmosphere+ocean $\mathrm{C}$ inventory, $p_{\mathrm{CO} 2}$ must fall. Conversely, when ocean $\mathrm{pH}$ is low $\left(\mathrm{H}^{+}\right.$concentration is high), then by Le Chatelier's principle, $\mathrm{C}$ in the ocean exists mostly as $\mathrm{CO}_{2} T$, and the fraction of total $\mathrm{C}$ in the atmosphere is large.

What sets $\mathrm{pH}$ ? Ocean $\mathrm{pH}$ rises when dissolved rock (e.g, $\mathrm{Ca}^{2+}, \mathrm{Na}^{+}$) is added to the ocean. That is because charge balance is maintained almost exactly in habitable oceans. Adding a mole of $\mathrm{Na}^{2+}$ to an ocean relieves one mole of $\mathrm{H}^{+}$from their duty of maintaining charge balance, so they revert to $\mathrm{H}_{2} \mathrm{O}$. Loss of $\mathrm{H}^{+}$raises $\mathrm{pH}$, and sucks $\mathrm{C}$ out of the atmosphere (Equation 1, Figure 4 . This effect of dissolved rock on $p_{\mathrm{CO} 2}$ does not require carbonate minerals to form. However, $\mathrm{C}$ can exist mostly as solid $\mathrm{CaCO}_{3}$ if $[\mathrm{Ca}]$ is high (or even as solid $\mathrm{Na}_{2} \mathrm{CO}_{3}$ minerals, if $[\mathrm{Na}]$ is extremely high). The relationship between $\mathrm{pH}$ and $p_{\mathrm{CO} 2}$ and $\mathrm{pH}$ is an equilibrium, and so it equally true to think of $p_{\mathrm{CO} 2}$ as driving $\mathrm{pH}$.

Assuming constant atmosphere+ocean C content, when $T$ rises ocean $\mathrm{pH}$ will fall. This fall is due to changes

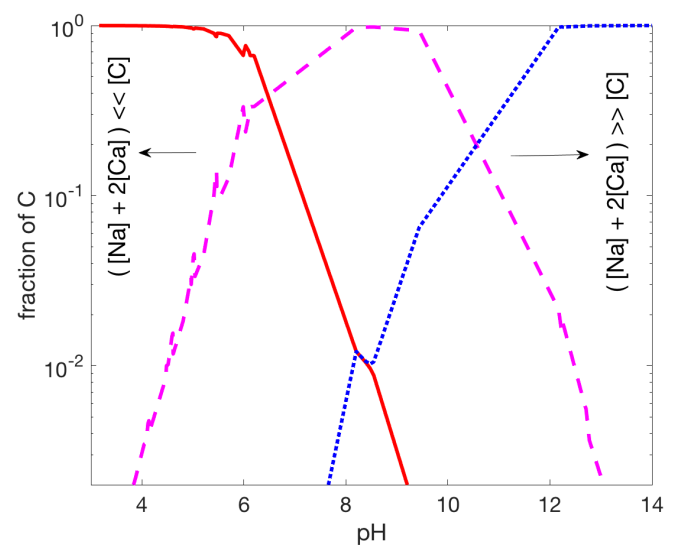

Figure 4. Showing $\mathrm{C}$ distribution within the aqueous phase (Bjerrum plot). The solid red line corresponds to the fractional abundance of dissolved $\mathrm{CO}_{2}$, the dashed magenta line corresponds to the fractional abundance of $\mathrm{HCO}_{3}^{-}$, and the dotted blue line corresponds to the fractional abundance of $\mathrm{CO}_{3}^{2-}$. Square brackets denote molalities; $\mathrm{Ca}$ and $\mathrm{Na}$ are examples of cations. Small wiggles in the curves are interpolation artifacts. $T=0.1^{\circ} \mathrm{C}$.

in $k_{H}$, the analogous equilibrium constants in Equation 2 , and increasing self-dissociation of water.

A general model for $p_{\mathrm{CO} 2}(t)$ would be complicated (Holland 1984, Hayes \& Waldbauer 2006, KrissansenTotton et al. 2018). Complexity occurs because $p_{\mathrm{CO} 2}$ is coupled to $\mathrm{pH}, \mathrm{pH}$ is affected by rock-water reaction, and the controls on rock-water reactions are complex and evolve with time. Fortunately, for waterworlds, the problem is simpler.

\section{4. $p_{\mathrm{CO} 2}(t)$ modeling is simplified by the waterworld approximation}

The waterworld climate evolution problem is simplified by five factors. Each factor tends to reduce the number of fluxes that we have to model. The cumulative effect of these five factors is to uncouple climate evolution from ocean-mantle geochemical cycling (Figure 6). The most important factor is that $\mathrm{C}$ exchange between the convecting mantle and the water ocean is limited on waterworlds - Factor \#4. This is because seafloor pressure on waterworlds curtails adiabatic decompression melting, which is the dominant mechanism of volcanism on rocky planets (Kite et al. 2009) (Figure 5) (Appendix A). The five factors, which we collectively term the "waterworld approximation," are as follows.

1. $\mathbf{H}_{2}$ is negligible.

2. Water loss to space is small.

3 . $\mathrm{H}_{2} \mathrm{O}$ in the atmosphere+ocean greatly outweighs $\mathrm{H}_{2} \mathrm{O}$ species in the silicate mantle.

4. C exchange between the deep mantle and the water ocean shuts down within $O\left(10^{8}\right)$ yr after the last giant impact.

5. No land.

We explain each factor below.

1. $\mathbf{H}_{2} / \mathbf{H e}$ is negligible. $\mathrm{H}_{2}$ blankets of the appropriate thickness to prolong habitability within the HZ of Kopparapu et al. (2013) require fine-tuning to form. They 
Table 1

Parameters and variables.

\begin{tabular}{|c|c|c|c|}
\hline Parameter & Description & Value & Units \\
\hline$a$ & semimajor axis & & $\mathrm{AU}$ \\
\hline$a_{s l}$ & anchor for the evolving snowline & 1.6 & AU \\
\hline$C$ & atmosphere+ocean $\mathrm{C}$ in $\mathrm{CO}_{2}$-equivalent & & $\mathrm{kg} \mathrm{m}^{-2}$ \\
\hline$c_{C}$ & $\mathrm{CO}_{2}$-equivalent $\mathrm{C}$ mass in atmosphere+ocean & & fraction of planet mass \\
\hline$c_{p, r}$ & specific heat of rock & & $\mathrm{J} \mathrm{kg}^{-1} \mathrm{~K}^{-1}$ \\
\hline$c_{p, f}$ & specific heat of aqueous fluid & 3800 & $\mathrm{~J} \mathrm{~kg}^{-1} \mathrm{~K}^{-1}$ \\
\hline$[\mathrm{C}],[\mathrm{Na}],[\mathrm{Ca}]$ & aqueous concentrations (molalities) of $\mathrm{C} ; \mathrm{Na} ; \mathrm{Ca}^{*}$ & & $\operatorname{mol}\left(\mathrm{kg} \mathrm{H}_{2} \mathrm{O}\right)^{-1}$ \\
\hline$f_{W}$ & $\begin{array}{l}\mathrm{H} \text { mass fraction of the atmosphere+ocean }+ \text { crust }+ \\
\text { (silicate mantle), excluding any } \mathrm{H} \text { in the core, } \\
\text { multiplied by } 9 \text { to get } \mathrm{H}_{2} \mathrm{O} \text {-equivalent mass fraction }\end{array}$ & & fraction of planet mass \\
\hline$f_{W, \max }$ & maximum $f_{W}$ in planetary embryos & & fraction of embryo mass \\
\hline $\mathrm{I} / \mathrm{E}$ & intrusive:extrusive volume ratio (lavas vs. sills/dikes) & & \\
\hline$k_{\text {cond }}$ & thermal conductivity of ocean & & $\mathrm{W} \mathrm{m}^{-1} \mathrm{~K}^{-1}$ \\
\hline$L_{*}$ & stellar luminosity at planet & & $\mathrm{W} \mathrm{m}-2$ \\
\hline$M_{p l}$ & planet mass & & Earth masses \\
\hline$P^{p i}$ & pressure & & $\mathrm{Pa}$, or bars \\
\hline$P_{l i t h}$ & pressure at the base of the lithosphere & & \\
\hline$p_{\mathrm{CO} 2}$ & partial pressure of atmospheric $\mathrm{CO}_{2}$ & & bars \\
\hline$R_{p l}$ & planet radius & & $\mathrm{km}$ \\
\hline$T, T_{\text {surf }}$ & temperature; ocean-surface temperature & & $\mathrm{K}$, or ${ }^{\circ} \mathrm{C}$ \\
\hline & silicate-mantle potential temperature & & \\
\hline$t^{P}$ & time & & Gyr \\
\hline$v_{\infty}$ & velocity at infinity (pre-giant-impact) & & $\mathrm{m} \mathrm{s}^{-1}$ \\
\hline $\mathrm{W} / \mathrm{R}$ & effective water/rock ratio of water/rock reactions & & - \\
\hline$z_{c r}$ & crust thickness & & \\
\hline$\Delta Z$ & ocean depth & & $\mathrm{km}$ \\
\hline$\Delta z_{b l}$ & thickness of conductively cooling layer(s) within ocean & & $\mathrm{km}$ \\
\hline$\tau_{c}$ & timescale for steam-atmosphere cooling & & Gyr \\
\hline$\tau_{x}$ & timescale for crust formation & & Gyr \\
\hline$\tau_{d y n}$ & timescale for $\mathrm{C}$ delivery & & Gyr \\
\hline$\tau_{h a b}$ & duration of habitable surface water & & Gyr \\
\hline$\tau_{\text {unmix }}$ & mixed-layer exsolution timescale & & Gyr \\
\hline$\rho_{c r}$ & crust density & 3000 & $\mathrm{~kg} \mathrm{~m}^{-3}$ \\
\hline$\rho_{w}$ & ocean density & 1300 & $\mathrm{~kg} \mathrm{~m}^{-3}$ \\
\hline
\end{tabular}

* We use mol/kg interchangeably with mol/ $\left(\mathrm{kg} \mathrm{H} \mathrm{H}_{2} \mathrm{O}\right)$, which introduces small errors that are acceptable for our purposes.

also swiftly escape to space (e.g. Wordsworth 2012 ; Owen and Mohanty 2016; Odert et al. 2018; but see Ramirez \& Kaltenegger 2017). While $\mathrm{H}_{2}$-rich low-density planets likely exist in the HZ (Rogers 2015; Van Eylen et al. 2018), we expect that they will have uninhabitably hot rock-volatile interfaces ("surfaces") (Rogers 2012) and we do not model them. We do not track $\mathrm{H}_{2}$ outgassing by $\mathrm{Fe}+\mathrm{H}_{2} \mathrm{O} \rightarrow \mathrm{FeO}+\mathrm{H}_{2}$. In the context of modern planet formation models, this reaction requires mixing of Fe with the volatile envelope during collisions between planets and planetary embryos. However, planetary embryos contain metal cores of $>500 \mathrm{~km}$ diameter, which merge quickly (Dahl \& Stevenson 2010, Jacobson et al. 2017, but see also Genda et al. 2017). We assume that the mantle is sufficiently oxidized that any volcanically outgassed $\mathrm{C}$ is in the form of $\mathrm{CO}_{2}$, and that $\mathrm{H}_{2}$ outgassing is minor. Therefore, we consider an $\mathrm{H}_{2}$-free, $\mathrm{CO}_{2}+\mathrm{H}_{2} \mathrm{O}\left( \pm \mathrm{N}_{2}\right)$ atmosphere.

2. Water loss to space is small, relative to the initial water complement, for waterworlds orbiting FGK stars. This is explained in Section 2.1.

3. $\mathbf{H}_{2} \mathrm{O}$ in the atmosphere+ocean outweighs " $\mathrm{H}_{2} \mathrm{O}$ " in the silicate mantle. Upon magmaocean crystallization shortly after the last giant impact, the silicate-magma ocean exsolves $\mathrm{H}_{2} \mathrm{O}$ (Elkins-Tanton 2011). The $\mathrm{H}_{2} \mathrm{O}$ concentration that is retained in the now-frozen magma (= silicate rock) is limited by the up- per mantle silicate minerals' $H$ storage capacity ${ }^{3}$ This storage capacity is small relative to the total water on waterworlds (Hirschmann 2006, Cowan and Abbot 2014, but see Marty 2012 for a contrary view). As a result, the water in the ocean greatly exceeds the water in the silicate mantle, so any subsequent cycling (e.g. Schaefer and Sasselov 2015; Komacek \& Abbot 2016, Korenaga et al. 2017) will have little (fractional) effect on ocean depth. Hypothetical rock-hydration feedbacks (e.g. Kasting and Holm 1992), if they are real, would also be limited by the small mantle storage capacity for H. Moreover, rockhydration feedbacks do not reduce ocean depth on worlds that have oceans much deeper than on Earth (Korenaga et al. 2017). For seafloor pressure $>10 \mathrm{GPa}$, the $\mathrm{H}_{2} \mathrm{O}$ storage capacity of mantle rock just beneath the stagnant lid jumps to $\sim 0.5-1.0$ wt\% (Ohtani et al. 2005). Even for such high seafloor pressures, however, the dominant $\mathrm{H}$ reservoir is still the ocean; this is because the $\mathrm{H}_{2} \mathrm{O}$ storage capacity of average mantle rock is still $\ll 0.5 \mathrm{wt} \%$. Moreover, in this paper we only consider $<8 \mathrm{GPa}$ seafloor pressures. To sum up, after the magma ocean has crystallized, ocean depth can be considered as

\footnotetext{
3 Assuming that the solid mantle convects sufficiently vigorously that each parcel of mantle passes through the upper mantle at least once (which is reasonable; Schubert et al. 2001), $\mathrm{H}_{2} \mathrm{O}$ contained in deeper hydrous phases will be rejected from the crystal during phase transition on ascent.
} 

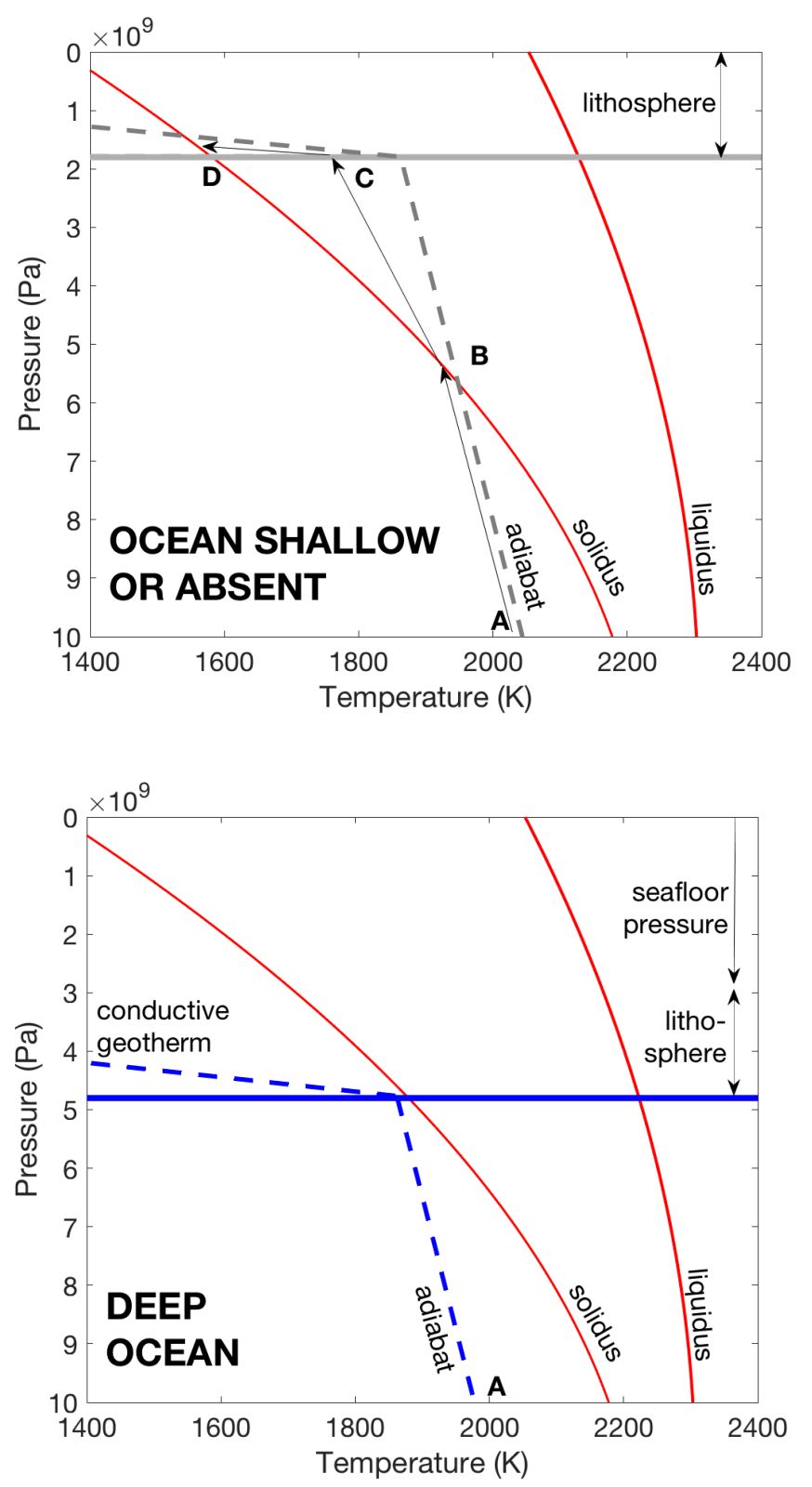

Figure 5. How the weight of water inhibits silicate volcanism on stagnant-lid waterworlds (and thus inhibits $\mathrm{C}$ exchange between the convecting mantle and the water ocean) (Kite et al. 2009). This figure is for stagnant-lid worlds, but similar arguments apply for plate tectonic worlds. In the upper panel, the arrowed thin line $\mathrm{A} \rightarrow \mathrm{B} \rightarrow \mathrm{C} \rightarrow \mathrm{D}$ tracks the partial (batch) melting of a parcel of initially solid mantle rock in the rising limb of a solid-state mantleconvection cell beneath a stagnant-lid lithosphere on a shallowocean world. From A $\rightarrow$ B, the path parallels the solid-state adiabat (dashed gray line). At B, melting begins, and increases until (at C) the parcel reaches the base of the $60 \mathrm{~km}$-thick stagnant lid (thick gray horizontal line). Further ascent (if any) is slow, and conductive cooling shuts down further melting (at D). For a stagnant-lid planet with a $3 \mathrm{GPa}$ ocean (bottom panel), no melting occurs. The dashed blue line is an adiabat displaced downwards, such that the sub-lithospheric temperature is the same as in the no-ocean case. For details, see Appendix A. a constant.

4. C exchange between the convecting mantle and the water ocean shuts down within $O\left(10^{8}\right)$ yr after the last giant impact. To shut down $\mathrm{C}$ cycling between a planet's mantle and ocean, it is necessary to shut down volcanic outgassing. For this to occur, it is sufficient to shut down volcanism. (Other modes of outgassing such as geysers are ultimately driven by volcanism). Volcanism is curtailed by seafloor pressure (Figure 5) (Kite et al. 2009). The seafloor pressure threshold needed to curtail volcanism depends on how the mantle convects, and is smaller for stagnant-lid convection ( 1-3 GPa). We analyze this effect in Appendix A. For simplicity, we impose an abrupt 1 GPa cutoff on volcanism. In reality some volcanism probably continues above this seafloor pressure, but (we assume) not enough to buffer atmosphere+ocean C. Because volcanism is intimately connected to plate tectonics, it is plausible that with little or no volcanism there is little or no tectonic resurfacing (Sleep 2000). Without tectonic resurfacing, the planet's mantle convects the same way as the mantles of Mars, Mercury, and the Moon: stagnant-lid worlds (O'Rourke \& Korenaga 2012, Noack et al. 2017, Dorn et al. 2018). In stagnant-lid mode, the mantle's convecting interior is cooled from above by conduction across a stable, very viscous boundary layer (the stagnant lid) (Stevenson 2003). We assume stagnant-lid volcanism in this paper, with little or no role for plate tectonics. Plate tectonics is considered in Section 6.5.

After volcanic outgassing has shut down, $\mathrm{C}$ is not released from the mantle into the ocean; neither is $\mathrm{C}$ subducted into the deep mantle, because no-volcanism stagnant-lid tectonics lacks subduction. Therefore, we treat the atmosphere+ocean+(shallow lithosphere) as a closed system with respect to $\mathrm{C}$ after $10^{8}$ yr (Figure 6).

Even without sustained volcanism, water and rock still react on waterworlds. Most importantly, volcanism will occur $\lesssim 10^{8}$ yr after the last giant impact, for example as the dregs of the magma ocean extrude. This volcanism is key to setting ocean chemistry, and is discussed in Section 3. Even after $\sim 10^{8} \mathrm{yr}$, slow alteration of porous seafloor rocks can continue, due to diffusion and/or changes in seafloor $T$ that shift mineral equilibria. This slow process, which we do not model, supplies chemical energy and nutrients (Section 6.4). Finally, carbonate precipitation and dissolution at (or just below) the seafloor may occur.

5. No land. Mountain-peak height is set by tectonic stress in competition with gravity-driven crustal spreading (Melosh 2011). The tallest peaks in the solar system are $\sim 20 \mathrm{~km}$ above reference level. Such peaks are drowned on waterworlds, so there is no land. With no land, there is no continental weathering.

Now, for a given planet mass $\left(M_{p l}\right)$, we can write

$$
p_{\mathrm{CO} 2}=p_{\mathrm{CO} 2}\left(f_{W, t=t_{0}}, c_{C, t=t_{0}},[X]_{t=t_{0}}, L_{*}(t), t\right)
$$

where $f_{W}$ is planet water mass fraction, $L_{*}$ is stellar luminosity, $[X]_{t=0}$ is initial ocean chemistry (including mineral phases in equilibrium with the ocean, if any), and $t$ is time in Gyr. The start time $t_{0}=0.1$ Gyr; our 


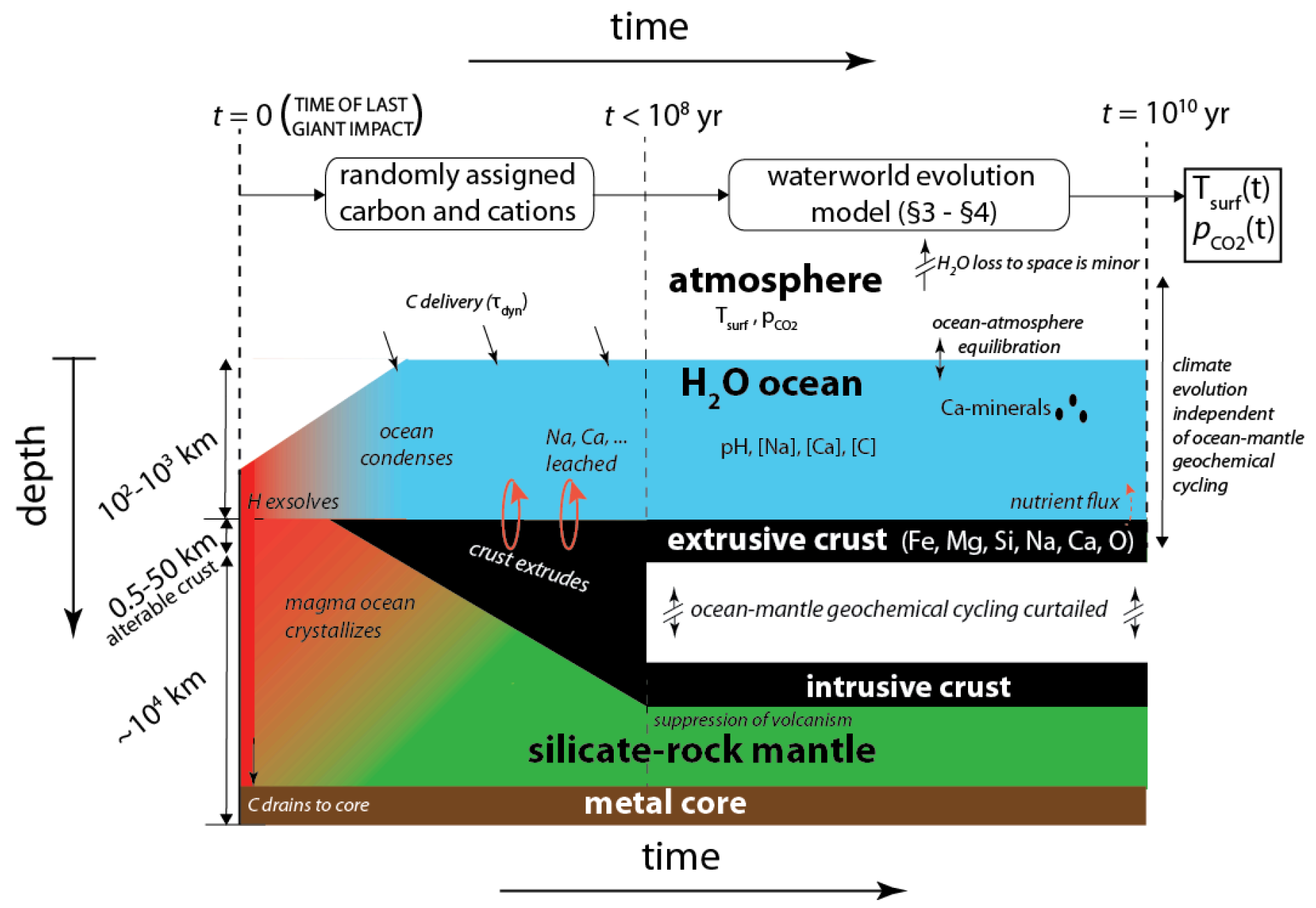

Figure 6. The flow of our waterworld evolution model. Seawater composition is set in the first $10^{8}$ yr (Section 3.1 ). Then, the atmosphere+ocean+(shallow crust) are isolated and the climate evolution is calculated (Section 3.2). Reservoirs (bold), processes (italics), and variables (regular text) that are discussed in the text are shown. Cycle-independent planetary habitability is enabled by cessation of ocean-mantle geochemical cycling after $\sim 10^{8}$ yr on waterworlds. See Section 6.4 for discussion of nutrient flux.

simulations have 0.1 Gyr timesteps (Figure 6). Continental weathering flux does not appear in Equation 3, because we assert "no land." Ocean-interior exchange flux does not appear in Equation 3, because we assert "no post-0.1 Ga $\mathrm{C}$ exchange between the deep mantle and the water ocean." (Figure 6). The only time dependence in Equation 3 is warming as the star brightens. If we can solve Equation (3) to get $p_{\mathrm{CO} 2}(t)$, we can find the corresponding surface temperature (from Figure 2) and thus find the duration of habitable surface water as the star brightens."

\section{DESCRIPTION OF THE WATERWORLD EVOLUTION MODEL}

We set out to solve Equation 3. The overall flow of our code is shown in Figure 6. Our code has two main stages:

- set initial seawater composition: we assign "initial" C abundance and cation abundance at random from cosmochemically/geophysically reasonable ranges $(t<0.1$ Gyr) (Section 3.1);
- compute $T_{\text {surf }}(t)$ and $p_{\mathrm{CO} 2}(t)(<0.1$ - $10 \mathrm{Gyr})$ (Section 3.2).

\subsection{Step 1: Set initial seawater composition}

In this subsection, we justify (1) treating the atmosphere+ocean $\mathrm{CO}_{2}$ mass fraction $\left(c_{C}\right)$ as a free parameter, varying between planets from $10^{-5}$ to $10^{-3}$ of planet mass, such that $c_{C} / f_{W}$ varies from $10^{-6}$ to $10^{-1}$; and (2) using salinities $O(0.1-1) \mathrm{mol} / \mathrm{kg}$. We define $c_{C}$ to be the mass of $\mathrm{C}$ in the atmosphere+ocean after the magma ocean has crystallized but before any carbonate formation (excluding $\mathrm{C}$ in the metal core or silicate mantle), divided by the mass of the planet, and multiplied by $44 / 12$ to get the $\mathrm{CO}_{2}$-equivalent mass fraction. In this paper we do not consider compositions that are very C-rich - comet-like. Comets made only a minor contribution to Earth's volatile budget (Marty et al.2016), but exoplanets might have comet-like compositions. Cometlike compositions require a more sophisticated treatment including clathrates and other C-rich phases (Bollengier et al. 2013, Levi et al. 2014; Marounina et al. 2017; Levi 
et al. 2017; Ramirez \& Levi 2018). Busy readers may skip to Section 3.2.

Seawater composition on stagnant-lid waterworlds is set by (1) delivery of $\mathrm{CO}_{2}$, and (2) supply of cations via water-rock reaction in the $10^{8} \mathrm{yr}$ after the last giant impact 4

(1) $C$ delivery. $\mathrm{C}$ dissolves into liquid Fe-metal in the immediate aftermath of giant impacts, when liquid silicate and liquid Fe-metal equilibrate (Kuramoto \& Matsui 1996, Hirschmann 2012, Dasgupta 2013). $\mathrm{C}$ is so iron-loving that, even if $2 \%$ of a $1 M_{\oplus}$ planet is composed of $\mathrm{C}$ (equivalent to $7 \mathrm{wt} \% \mathrm{CO}_{2}$ !), only $<100$ ppm escapes the core (Figure 2 in Dasgupta 2013). The lure of the Fe-metal core for $\mathrm{C}$ was so strong that most of the $\mathrm{C}$ that existed in the Earth prior to the Moon-forming impact is believed to have been trapped in the core during the magma ocean era associated with giant impacts. Therefore, the $\mathrm{C}$ in our bodies (and in Earth's ocean) is thought to represent C delivered after the last giant impact (Bergin et al. 2015). C's behavior under giant impact conditions contrasts with that of H (Kuramoto \& Matsui 1996). H does not partition as strongly into liquid Fe-metal under giant impact conditions as does C. Therefore, in contrast to $\mathrm{C}, \mathrm{H}$ now in Earth's oceans could have been delivered to the Earth prior to the last giant impact. Indeed, the H in Earth's oceans is thought to have arrived during the main stage of planet assembly (O'Brien et al. 2014; Rubie et al. 2015. O'Brien et al. 2018; see Albarède 2009 for an opposing view). Giant impacts are stochastic, so the ratio of $\mathrm{CO}_{2}-$ equivalent $\mathrm{C}$ mass in the atmosphere+ocean to the planet water content $\left(c_{C} / f_{W}\right)$ is expected to vary stochastically (Hirschmann 2016) (Section 6.5). Another source of variation in $c_{C} / f_{W}$ is the varying extent (which we do not track) of the reaction $\mathrm{Fe}+\mathrm{H}_{2} \mathrm{O} \rightarrow \mathrm{FeO}+\mathrm{H}_{2}$. Given these uncertainties, we vary $c_{C}$ from $10^{-5}$ to $10^{-3}$, such that $c_{C} / f_{W}$ varies from $10^{-6}$ to $\sim 10^{-1}$. $c_{C}=10^{-5}$ corresponds to an Earth/Venus-like $\mathrm{C}$ abundance, and we find that $c_{C}=10^{-3}$ all but ensures a $p_{\mathrm{CO} 2}>40 \mathrm{bar}$ atmosphere, which we deem uninhabitable.

(2) Supply of cations via water-rock reaction: Rock with the composition of Earth's oceanic crust $(\mathrm{CaO} \approx$ $\mathrm{MgO} \approx \mathrm{FeO} \approx 10 \mathrm{wt} \%$, Gale et al. 2013) can form $\{\mathrm{Ca}, \mathrm{Mg}, \mathrm{Fe}\} \mathrm{CO}_{3}$ minerals, and potentially trap up to 70 (bars $\left.\mathrm{CO}_{2}\right) /(\mathrm{km}$ crust). To what extent is this potential realized? The fluid composition resulting from waterrock reactions $<10^{8}$ yr after the last giant impact can be estimated (a) by analogy to similar worlds, (b) with reaction-transport models, and (c) by geophysical rea-

\footnotetext{
4 Volatiles initially in the steam atmosphere are less important. To see this, consider that immediately after the last giant impact, volatiles and magma are in equilibrium (Zahnle et al. 1988 Abe et al. 2000 Zahnle et al. 2015). When the surtace cools below $\sim 1600 \mathrm{~K}$, the surtace can crust over. Volatiles that are in the atmosphere at this point might be taken up by the $\mathrm{H}_{2} \mathrm{O}_{(l)}$ ocean after further cooling. The most abundant non- $\mathrm{CO}_{2}$ non- $\mathrm{H}_{2} \mathrm{O}$ volatile in a 100-bar, $1600 \mathrm{~K}$ steam-atmosphere is $\mathrm{HCl}$ according to the calculations of (Lupu et al. 2014). $\mathrm{HCl}$ volume mixing ratio $\approx 10^{-2}$, i.e., small. If this atmosphere condenses into a hypothetical $100 \mathrm{~km}$-deep ocean (the shallowest we consider), we obtain $[\mathrm{Cl}] \approx 10^{-2} \mathrm{~mol} / \mathrm{kg}$, which is small. Na species are even less abundant in the steam atmosphere (e.g. (Schaefer et al. 2012)), and $\mathrm{Na}$ is more abundant in the planet as a whole, and is readily leached into the ocean.
}

soning about the mass of rock available for water-rock reactions.

(2a) Previous work on extraterrestrial oceans such as Europa and Enceladus - suggests salinities $O(0.1-1) \mathrm{mol} / \mathrm{kg}$. For example, Glein et al. (2015) and Hand \& Chyba (2007) estimate salinity using spacecraft data; and McKinnon \& Zolensky (2003), Zolotov (2007), and Zolotov \& Kargel (2009), estimate salinity using models.

(2b) We use the aqueous geochemistry thermodynamics solver CHIM-XPT (Reed 1998) to explore reactions between water and rock (Appendix D). Our rock input for these runs has the composition of Earth's oceanic crust (Gale et al. 2013). Rocks release cations into the ocean that - even when not forming carbonates - can control ocean chemistry. Rock-sourced cations (e.g. $\mathrm{Na}^{+}$ and $\mathrm{Ca}^{2+}$ ) substitute in the ocean charge balance for $\mathrm{H}^{+} . \mathrm{H}^{+}$removal raises $\mathrm{pH}$, which in turn raises the fraction of dissolved inorganic carbon that is stored as $\mathrm{HCO}_{3}$ and $\mathrm{CO}_{3}^{2-}$ rather than as dissolved $\mathrm{CO}_{2}$ (Figure 4). As dissolved $\mathrm{CO}_{2}$ decreases, $p_{\mathrm{CO} 2}$ decreases in proportion (by Henry's Law). Two cations, $\mathrm{Na}^{+}$and $\mathrm{Ca}^{2+}$, are used in our waterworld evolution model. Other cations contribute little to the charge balance of outlet fluid in our CHIM-XPT runs (Appendix D), and so are ignored. CHIM-XPT runs (for $T \leq 600 \mathrm{~K}$ ) indicate that $\mathrm{Mg}$ and $\mathrm{Fe}$ likely form silicates, not carbonates, and so $\mathrm{Mg}$ and $\mathrm{Fe}$ do not draw down $\mathrm{CO}_{2}$. Non-participation of $\{\mathrm{Mg}, \mathrm{Fe}\}$ cuts $\mathrm{C}$ uptake by a factor of $\sim 3 . \mathrm{CaCO}_{3}$ forms, and buffers $[\mathrm{C}]$ in outlet fluid to $\lesssim 0.1 \mathrm{~mol} / \mathrm{kg}$. Complete use of Ca to form $\mathrm{CaCO}_{3}$, with only minor $\mathrm{MgCO}_{3} / \mathrm{FeCO}_{3}$, matches data for carbonated Archean seafloor basalts (Nakamura \& Kato 2004, Shibuya et al. 2012, 2013).

(2c) Geophysical arguments about the mass of rock available to react with fluid may be divided into: (i) eruption history arguments, and (ii) pervasiveness arguments. We assume that rock-water reactions occur at $T_{\text {seafloor }}<650 \mathrm{~K}$ (for reasons given in Appendix $\mathrm{C}$ ).

(i) Eruption history: Alteration by water is much easier for extrusive rocks (lavas) than for intrusive rocks (sills and dikes). That is because intrusives have $\gtrsim 10^{3}$-fold lower permeability, which denies entry to altering fluids (Fisher 1998, Alt 1995). Intrusive:extrusive ratios (I/E) on Earth are scattered around an average of $\sim 5: 1$ (6.5:1 for oceanic crust) (White et al. 2006: White \& Klein 2014). Extrusives less than $0.5 \mathrm{~km}$ below the seafloor - corresponding to the highest permeabilities in oceanic crust - are the site of most of the alteration within the oceanic crust on Earth. Altered crust on an exoplanet could be much thicker than $0.5 \mathrm{~km}$ if the extrusive pile were thicker. A thicker extrusive pile is likely for stagnant lid mode. In this mode, each lava erupts at the surface, cools and is rapidly altered, and then is buried by later lavas. Extrusives whose alteration leaves them completely leached of an element will give a waterworld ocean with an aqueous molality (denoted by square brackets) of

$$
\frac{23}{1000}[\mathrm{Na}]=\mathrm{Na}^{\prime} \frac{z_{c r} \rho_{c r}}{z_{o c} \rho_{w}}(\mathrm{I} / \mathrm{E})^{-1}
$$

where $z_{O c}$ is ocean thickness, $z_{c r}$ is crust thickness, $\mathrm{Na}^{\prime}=0.028$ is the $\mathrm{Na}$ mass concentration in the crust (Gale et al.2013), $\rho_{c r}=3 \times 10^{3} \mathrm{~kg} \mathrm{~m}^{-3}$ is crust density, 
and $\rho_{w} \sim 1300 \mathrm{~kg} \mathrm{~m}^{-3}$ is ocean density (this is the density of water at $1.8 \mathrm{GPa}$ and $373 \mathrm{~K}$; Abramson \& Brown 2004 ). For $z_{o c}=100 \mathrm{~km}, z_{c r}=50 \mathrm{~km}$, and I/E $=5$, this gives $[\mathrm{Na}]=0.3 \mathrm{~mol} / \mathrm{kg}$.

(ii) Pervasiveness: Earth's modern oceanic crust is altered mainly near veins and cracks (Alt 1995). However, more pervasive alteration of seafloor extrusive rocks took place under C-rich, perhaps warmer climates $\sim 3.4 \mathrm{Ga}$ on Earth (Nakamura \& Kato 2004). Moreover, Earth's ocean $\mathrm{pH}$ is thought to have been moderate for $4 \mathrm{Ga}$ $(\mathrm{pH}=7.5 \pm 1.5 ;$ Halevy \& Bachan 2017; KrissansenTotton et al. 2018), and either higher $\mathrm{pH}$ or lower $\mathrm{pH}$ would be more corrosive. Finally, water is highly corrosive as a supercritical fluid, and seafloor $T$ and $P$ on waterworlds can reach supercritical conditions (Figure 3). Therefore, a wide range of alteration - including pervasive alteration - is possible on other worlds (Vance et al. 2007, 2016, Kelemen \& Hirth 2012, Neveu et al. 2015).

Taken together, (2a)-(2c) suggest that, for waterworlds,

- both $\mathrm{Na}$ and $\mathrm{Ca}$, but not $\mathrm{Fe}$ and $\mathrm{Mg}$, participate in $\mathrm{CO}_{2}$ drawdown if rocks interact with water;

- the reasonable range of rock-layer thicknesses for complete/pervasive interaction with water is $0.1 \mathrm{~km}$ (Earth-like) to $50 \mathrm{~km}$. The biggest value corresponds to an unusually low $\mathrm{I} / \mathrm{E}=1: 1$, pervasive alteration of extrusives, and a crust thickness of $100 \mathrm{~km}$, which is large relative to models and relative to solar system data (Taylor \& McLennan 2009; O'Rourke \& Korenaga 2012; Plesa et al. 2014; Tosi et al. |2017). The largest values might be thought of as including weathering of impactors and meteoritic dust. For all but the very largest impacts, impact-target materials (Sleep \& Zahnle 2001) will not contribute cations, because the ocean will shield the seafloor from impacts. The only ejecta will be water and impactor materials (Nimmo et al. 2008, Marinova et al. 2008).

The corresponding cation abundances (for a $100 \mathrm{~km}$-deep ocean, and neglecting Ca-mineral formation) are $0.003-$ $1.4 \mathrm{~mol} / \mathrm{kg} \mathrm{Na}$, and $0.008-4 \mathrm{~mol} / \mathrm{kg} \mathrm{Ca}$. Lumping $\mathrm{Na}$ and $\mathrm{Ca}$ as moles of equivalent charge (Eq), we obtain $0.02-9 \mathrm{Eq} / \mathrm{kg}$.

\subsection{Step 2: Compute $T_{\text {surf }}(t)$ and $p_{\mathrm{CO} 2}(t)$}

$p_{\mathrm{CO} 2}(t)$ is set by sea-surface equilibration between the well-mixed ocean and the atmosphere (Figure 6). This equilibrium is rapid compared to the time steps in our model.

To find $\mathrm{pH}\left(T_{\text {surf }}\right)$ and $p_{\mathrm{CO} 2}\left(T_{\text {surf }}\right)$, for each of a wide range of prescribed seawater cation content and dissolved inorganic $\mathrm{C}$ content, we use CHIM-XPT. We found (batch) equilibria in the system $\mathrm{H}_{2} \mathrm{O}-\mathrm{HCO}_{3}^{-}-\mathrm{Ca}^{2+}-\mathrm{Na}^{+}$, including formation of calcite $\left(\mathrm{CaCO}_{3}\right)$ and portlandite $\left(\mathrm{Ca}(\mathrm{OH})_{2}\right)$.

We consider the full range of geophysically plausible cation contents. In Section 4 , we initially describe results for 3 cation cases: $(1)[\mathrm{Na}] \approx 0.5 \mathrm{~mol} / \mathrm{kg},[\mathrm{Ca}] \approx 0$ - higher-pH, but lacks minerals; $(2)[\mathrm{Ca}] \approx[\mathrm{Na}]=0-\mathrm{a}$ lower-pH case; $(3)[\mathrm{Ca}]=0.25 \mathrm{~mol} / \mathrm{kg},[\mathrm{Na}] \sim 0$ - higher$\mathrm{pH}$. Many of the runs in case (3) form calcite (or, for $\mathrm{pH}$
$>9$, portlandite). We do not track the fate of Ca-mineral grains, which store most of the $\mathrm{Ca}$ in our higher-pH equilibrium model output. Depending on ocean depth and grain size, minerals might stay suspended in the water column, sink and redissolve, or pile up on the seafloor. Whatever the fate of individual grains, equilibrium with calcite buffers shallow-ocean (and thus atmosphere) $\mathrm{CO}_{2}$ content for ocean chemistries that form calcite.

To find $\mathrm{CO}_{2}$ solubilities, we used tables of $\mathrm{CO}_{2}$ solubility in pure $\mathrm{H}_{2} \mathrm{O}$, fit to experimental data. Specifically, we interpolated and extrapolated in Table 3 of Carroll et al. (1991) and Table 3 of Duan \& Sun (2003), also using the constraint that $\mathrm{CO}_{2}$ solubility $=0$ when $p_{a t m}=p_{\mathrm{H} 2 \mathrm{O}}$. For $p_{\mathrm{H} 2 \mathrm{O}}$, we used the formulation in Appendix B of Duan \& Sun (2003). $\mathrm{CO}_{2}$ solubility is reduced by salts. For a $1 \mathrm{~mol} / \mathrm{kg}$ solution at $10 \mathrm{bar}$ and $303 \mathrm{~K}$, salting-out reduces solubility by $18 \%$ (Duan \& Sun 2003). However, we do not include the direct effect of $\mathrm{Na}^{+}$or $\mathrm{Ca}^{2+}$ on $\mathrm{CO}_{2}$ solubility in our model, reasoning that the $\mathrm{pH}$ effect is more important and that the range of $\mathrm{C}$ considered in our model is so large that the effect of the ions on $\mathrm{CO}_{2}$ solubility is less important. Using the $\mathrm{CO}_{2}$ solubilities and the carbonate system equilibria from the CHIM-XPT calculations, we compute $p_{\mathrm{CO} 2}\left(T_{\text {surf }},[\mathrm{Na}],[\mathrm{Ca}],[\mathrm{C}]\right)$. Equipped with $p_{\mathrm{CO} 2}\left(T_{\text {surf }},[\mathrm{Na}],[\mathrm{Ca}],[\mathrm{C}]\right)$, we next calculate (for a given planet surface gravity, and fixing atmospheric molecular mass $=44 \mathrm{~g} /$ mole $) p_{\mathrm{CO} 2}\left(T_{\text {surf }},[\mathrm{Na}],[\mathrm{Ca}], c_{C}\right)$. Here, $c_{C}$ is the atmosphere+ocean column $\mathrm{C}$ abundance and can (in principle) greatly exceed the ocean C-storage capability. To ameliorate interpolation artifacts, we smooth out each $p_{\mathrm{CO} 2}\left(T_{\text {surf }}\right)$ curve using the MATLAB smooth function with a $30{ }^{\circ} \mathrm{C}$ full-width bandpass. (Appendix E has more details about how ocean-atmosphere equilibration is calculated).

Next, we interpolate the $T_{\text {surf }}\left(L_{*}, p_{\mathrm{CO} 2}\right)$ results of a $1 \mathrm{D}$ radiative-convective climate model (Wordsworth \& Pierrehumbert 2013a). Their study neglects clouds, assumes a fixed relative humidity of 1.0 , and adjusts the surface albedo to a value (0.23) that reproduces present-day Earth temperatures with present-day $\mathrm{CO}_{2}$ levels. (Comparison to the results of models that include clouds, and omit the albedo contribution of bright continents, indicates that this assumption will not affect the qualitative trends presented in the current paper; see Section 6.5.) We extrapolate the log-linear fit to $p_{\mathrm{CO} 2}=3 \times 10^{-6}$ bar. $T_{\text {surf }}$ always increases with $L_{*}$. $T_{\text {surf }}$ usually increases with $p_{\mathrm{CO} 2}$ as well, except near the outer edge of the $\mathrm{HZ}\left(p_{\mathrm{CO} 2}>8\right.$ bars in Figure 7). Near the outer edge of the habitable zone, adding $\mathrm{CO}_{2}$ leads to cooling because the Rayleigh scattering of incoming shortwave radiation from extra $\mathrm{CO}_{2}$ exceeds the greenhouse warming from extra $\mathrm{CO}_{2}$. Where Wordsworth \& Pierrehumbert (2013a) find multiple stable equilibria in their $1 \mathrm{D}$ atmospheric radiativeconvective model, we use the warmer of their two solutions, on the assumption that impacts intermittently allow the atmosphere+(wave-mixed ocean) (which have relatively low thermal inertia) to jump onto the warm branch. Once the atmosphere+(wave-mixed ocean) have reached the warm branch, the deep ocean will gradually adjust to the new, higher temperature. To find oceanatmosphere equilibria, we find $\left\{T_{\text {surf }}, p_{\mathrm{CO} 2}\right\}$ combina- 
tions that satisfy $L_{*}(t),[\mathrm{Na}]$, and [Ca] (Figure 7), as explained in Section 4.1. Although the ocean $T$ varies with depth within the ocean (Figure 3), the $\mathrm{CO}_{2}$ solubility increases with depth, and so the ocean surface temperature $T_{\text {surf }}$ is the temperature that matters for the purpose of finding ocean-atmosphere equilibria. (Appendix E gives more details, and shows the results of a sensitivity test using the $1 \mathrm{D}$ radiative-convective climate model of Ramirez et al. 2014).

At each timestep, we check for ice. Ice at the ocean surface (ice I) will have steady-state thickness $<20 \mathrm{~km}$ (due to geothermal heat). We assume initially ice-covered surfaces have an albedo that declines due to dark exogenic contaminants (meteoritic dust) on a timescale $\ll 10 \mathrm{Gyr}$, back to albedo $=0.23$. This value is used for convenience, as it is the surface+clouds albedo used by (Wordsworth \& Pierrehumbert 2013a). This lowering of initially high albedo is reasonable because, in our model, planets develop surface ice not at all, or very early. Early-developed ice cover will sweep up dark planet-formation debris (Löhne et al. 2008). The low albedo of debris is one of the reasons that all icy worlds in the solar system have dark surfaces except terrains which have been tectonically resurfaced and/or created after the stage of high impact flux in the early solar system. Lower albedo allows stellar luminosity increase to cause ice-covered surfaces to melt (Section 6.5). Melting with these assumptions always leads to a habitable state (this need not be true if $\mathrm{CO}_{2}$ outgassing is allowed; Yang et al. 2017, Turbet et al. 2017). If ice cover develops later in planetary history, then initially high albedos can stay high, but this is very uncommon in our model. In addition to checking for surface ice, we also evaluate whether high-pressure ice phases (ice VI, ice VII) are stable at any depth between the ocean surface and the seafloor along a pure-liquid$\mathrm{H}_{2} \mathrm{O}$ adiabat (Appendix B, Figure 3). We do not track the climate or $\mathrm{pH}$ in detail when $\mathrm{HP}$-ice is present (see Levi \& Sasselov 2018), and in particular we do not redistribute $\mathrm{CO}_{2}$ between clathrates, the ocean, and the atmosphere. This is because we conservatively assume that if HP-ice is present, the world is not habitable.

\section{RESULTS OF WATERWORLD EVOLUTION MODEL}

All of the results presented here are for a Sun-like (Gtype) star with (log luminosity)-versus-time fit to a standard solar model (Bahcall et al. 2001). For this model, $L_{*}$ increases by $\sim 8 \% \mathrm{Gyr}^{-1}$. We find that:

- waterworld climate is usually stable when $T$ is perturbed (Section 4.1);

- habitable surface water can persist for many Gyr (Section 4.2);

- using geologically and cosmochemically plausible priors in a parameter sweep, we estimate that $\sim 1 / 4$ of waterworlds have habitable surface water for $>1$ Gyr (Section $4.3)$.

\subsection{How individual waterworlds evolve}

Charting planet evolution. With the waterworld approximation, the cations available to the ocean are constant with time after 0.1 Gyr. Thereafter, $T_{\text {surf }}$ and the total amount of $\mathrm{C}$ in the atmosphere+ocean (including $\mathrm{C}$ in Ca-minerals, if any) are the sole controls on $p_{\mathrm{CO} 2}$. $\quad p_{\mathrm{CO} 2}$ usually increases with $T_{\text {surf }}$, due to the low solubility of $\mathrm{CO}_{2}$ in warm water, and related $T$-dependent shifts in the carbonate system equilibria (Figure 7). $p_{\mathrm{CO} 2}=p_{\mathrm{CO} 2}\left(T_{\text {surf }}\right)$ is a single-valued function for any cation and total-C concentration (Figure 7 ). $T_{\text {surf }}=T_{\text {surf }}\left(p_{\mathrm{CO} 2}\right)$ greenhouse curves (green lines) can be computed using a climate model given semimajor axis $a$ and $L_{*}(t)$. To find the $p_{\mathrm{CO} 2}$ and $T_{\text {surf }}$ for a given time, we locate the green line corresponding to that star age, then find its lowest- $T_{\text {surf }}$ stable intersection with the appropriate $p_{\mathrm{CO} 2}\left(T_{\text {surf }}\right)$ line (Figure 7 ).

As $L_{*}$ increases with time (Bahcall et al. 2001), $T_{\text {surf }}=T_{\text {surf }}\left(p_{\mathrm{CO} 2}, L_{*}\right)$ increases for a given $p_{\mathrm{CO} 2}$. This is shown by the rightward drift of the green lines (greenhouse curves) in Figure 7. The green lines are closely spaced at $p_{\mathrm{CO} 2} \sim 0.2-20$ bars, corresponding to a small $T_{\text {surf }}$ rise for a given increase in $L_{*}$ (Figure 2). This prolongs the duration of habitable surface water (longer $\left.\tau_{h a b}\right)$. By contrast the green lines are widely spaced at low $p_{\mathrm{CO} 2}$, so a small increase in $L_{*}$ corresponds to a rapid $T_{\text {surf }}$ rise (Figure 2). The $T_{\text {surf }}$ increase is usually more than would occur without ocean chemistry (Kitzmann et al. 2015), because the greenhouse gas $\mathrm{CO}_{2}$ is usually exsolved with increasing $T_{\text {surf }}$. For a given total atmosphere+ocean $\mathrm{C}$ inventory (e.g., $1.1 \times 10^{6} \mathrm{~kg} / \mathrm{m}^{2}$ $\mathrm{CO}_{2}$-equivalent), the $p_{\mathrm{CO} 2}$ is much higher (for a given $T_{\text {surf }}$ ) for the cation-poor case relative to the cation-rich cases. This is due to the higher $\mathrm{pH}$ of the cation-rich cases (Figure 4). However, $p_{\mathrm{CO} 2}$ increases more steeply with $T_{\text {surf }}$ for the cation-rich cases.

For most waterworld-evolution tracks, each time step is independent of the previous ones. Therefore, the colored portions of the waterworld evolution tracks (ice-free oceans) in Figures 810 should be valid whether or not $\mathrm{HP}$-ice is present earlier in that world's history (gray portions of the lines).

Stability and instability. To see that $T_{\text {surf }}-p_{\mathrm{CO} 2}$ equilibria are usually stable, consider the point around $50^{\circ} \mathrm{C}$, 1 bar. Suppose we add some energy to the system, perturbing $T_{\text {surf }}$. This drives $\mathrm{CO}_{2}$ from the ocean to the atmosphere - moving along the blue line - which will cause more warming, leading to a positive feedback on the initial perturbation. However, at fixed $L_{*}$, the value of $p_{\mathrm{CO} 2}$ that is in equilibrium with the increased surface temperature is even higher than the increased $\mathrm{CO}_{2}$. Therefore, the climate is stable.

For $P>1$ bar, exsolving $\mathrm{CO}_{2}$ can be a negative (stabilizing) feedback on climate evolution. For example, consider the point at $40^{\circ} \mathrm{C}, 10$ bars in Figure 7. An increase in $L_{*}$ corresponding to 1 Gyr of stellar evolution at constant $p_{\mathrm{CO} 2}$ would cause a $T_{\text {surf }}$ increase of $\sim 40 \mathrm{~K}$. However, the climate is constrained to evolve along a curve of constant seawater cation abundance. Therefore, $\mathrm{CO}_{2}$ is exsolved from the ocean. Cooling from Rayleigh scattering per unit of added $\mathrm{CO}_{2}(\mathrm{~g})$ exceeds greenhouse warming per unit of added $\mathrm{CO}_{2(\mathrm{~g})}$, so adding $\mathrm{CO}_{2(\mathrm{~g})}$ cools the planet (Ramirez et al. 2014). For the purple dashed line, the $T_{\text {surf }}$ increase is only $<10 \mathrm{~K}$; a negative feedback. This negative feedback is not a geochemical cycle.

$\mathrm{CO}_{2}$ 's solubility in water (for fixed $p_{\mathrm{CO} 2}$ ) increases for $T_{\text {surf }}>(110-160)^{\circ} \mathrm{C}$. This feedback is mildly stabilizing, but we neglect $\partial p_{\mathrm{CO} 2, \mathrm{GH}} / \partial T<0$ in the following discussion as these cases are rarely important. 

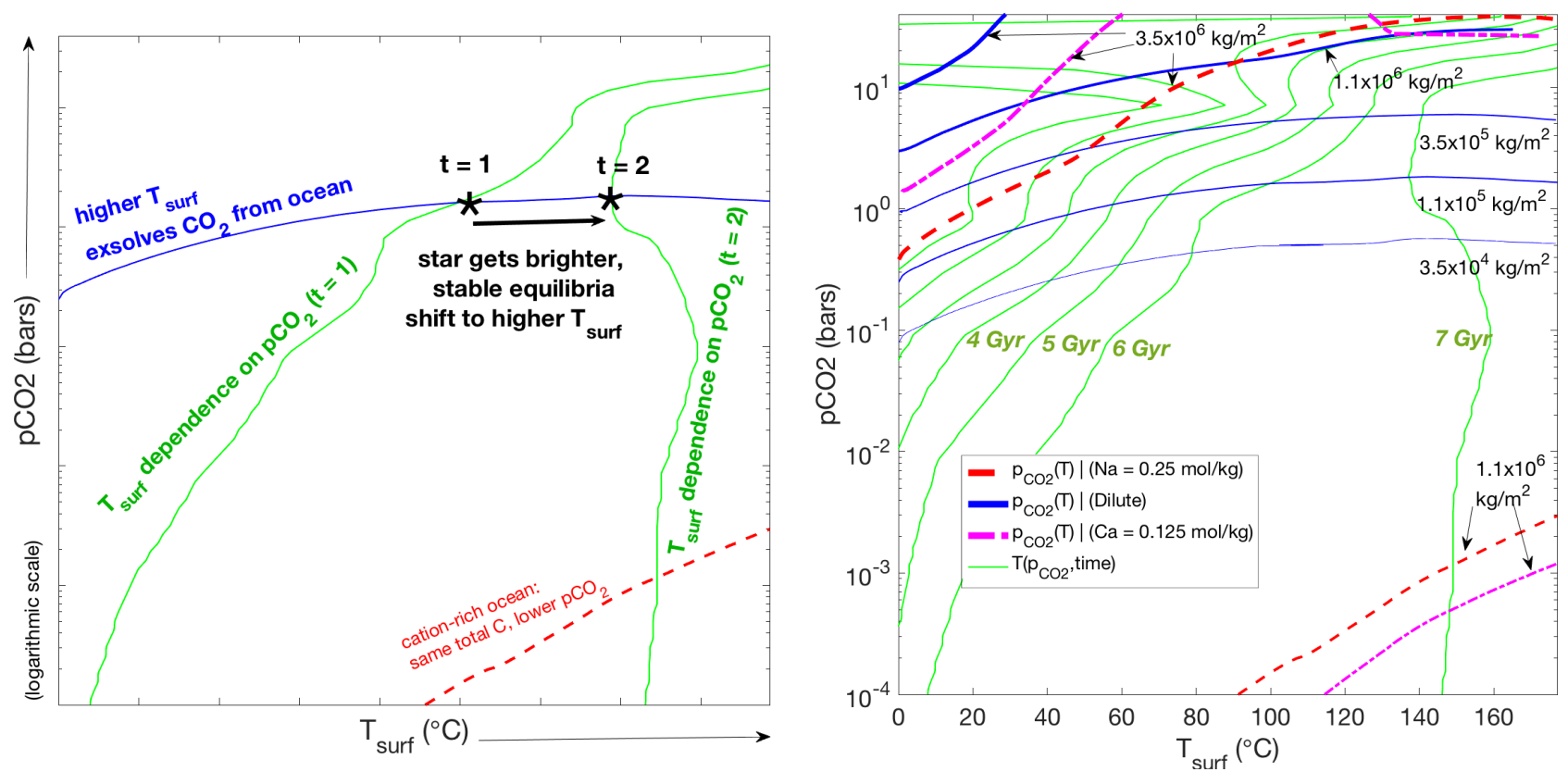

Figure 7. Left panel: scheme for tracking equilibria between ocean chemistry and atmospheric $p_{\mathrm{CO} 2}$. Equilibria shift as the insolation increases with host-star age on the main sequence $(t)$. Right panel: chart for finding equilibria between ocean chemistry and atmospheric $p_{\mathrm{CO} 2}$ (planet water mass fraction $=0.01, a=1.1 \mathrm{AU}$ ). Green lines correspond to $p_{\mathrm{CO} 2}\left(T_{\text {surf }}\right)$ for fixed $L_{*}$, with $T_{\text {surf }}$ increasing as $L_{*}$ rises with star age on the main sequence (interpolated from Wordsworth \& Pierrehumbert 2013a small wiggles are interpolation artifacts). The green lines are seperated at intervals of $1 \mathrm{Gyr}$ in star age on the main sequence. The blue, red, \& magenta lines correspond to $p_{\mathrm{CO} 2}$ as a function of $T_{\text {surf }}$, for different assumptions about ocean cation content. The solid blue lines correspond to a cation-poor ocean, the dashed red lines correspond to a $[\mathrm{Na}]=0.25 \mathrm{~mol} / \mathrm{kg}$ ocean, and the dash-dot magenta lines correspond to a $[\mathrm{Ca}]=0.125 \mathrm{~mol} / \mathrm{kg}$ ocean. Line thickness increases with equal amounts of total atmosphere+ocean $\mathrm{C}$ (including $\mathrm{C}$ in Ca-minerals, if any). Lines of the same thickness have the same total atmosphere+ocean C: $\left\{3.5 \times 10^{4}, 1.1 \times 10^{5}, 3.5 \times 10^{5}, 1.1 \times 10^{6}, 3.5 \times 10^{6}\right\} \mathrm{kg} \mathrm{m}^{-2} \mathrm{CO}_{2}$-equivalent $\mathrm{C}$ are considered.

For a $\left\{p_{\mathrm{CO} 2}, T_{\text {surf }}\right\}$ point to undergo runaway warming in response to small $T_{\text {surf }}$ increases, two conditions must be satisfied. (1) $\partial p_{\mathrm{CO} 2, \mathrm{OC}} / \partial T>\partial p_{\mathrm{CO} 2, \mathrm{GH}} / \partial T$, where the subscript oc refers to ocean chemistry curves, and the subscript $\mathrm{GH}$ refers to greenhouse curves. Otherwise, the positive feedback has finite gain, and there is no runaway. (2) $\partial p_{\mathrm{CO} 2, \mathrm{GH}} / \partial T>0$. If (2) is not satisfied, then there is a stabilizing, negative feedback.

Exsolution-driven climate instabilities (runaway feedbacks) are possible in the model. For example, consider the red thick-dashed line corresponding to $\mathrm{Na}=0.25 \mathrm{~mol} / \mathrm{kg}, c_{C}=3.5 \times 10^{6} \mathrm{~kg} \mathrm{~m}^{-2}$ in Figures 8 and 9. At 2.0 Gyr and 1.1 AU, two stable states are possible for these inputs: $\left\{37^{\circ} \mathrm{C}, \sim 2\right.$ bars $\}$ and $\left\{75^{\circ} \mathrm{C}\right.$, $\sim 10$ bars $\}$ (Figure 7). As $L_{*}$ increases, both stable states get warmer. Then, at $2.3 \mathrm{Gyr}$, the $\mathrm{GH}$ curve no longer intersects the thick red dashed line for $T_{\text {surf }}<60^{\circ} \mathrm{C}$. The cool branch has disappeared; as a result, the climate suddenly warms. (Emergence of cold solutions as solar luminosity increases is much less common in our model.) Because of interpolation artifacts and our very approximate treatment of $\mathrm{CO}_{2}$ solubility, one should be careful about reading out quantitative gradients for specific data points from the plots. The rate of change associated with these instabilities is unlikely to risk whole-ocean microbial extinction (in our opinion), provided that both the initial climate and final climate are habitable. The model-predicted instabilities are discussed further in Appendix F.

Duration of habitable surface water $\left(\tau_{h a b}\right)$. In some cases, habitability can persist for many Gyr (Figures 8 and 9 . As $L_{*}$ rises, either $T_{\text {surf }}$ eventually exceeds $450 \mathrm{~K}$, or
$\mathrm{CO}_{2}$ exceeds $\sim 40$ bars, either of which we deem sufficient to extinguish surface habitability (for a discussion, see Section 6.4). Different cation cases with the same $\mathrm{CO}_{2}$-equivalent atmosphere+ocean $\mathrm{C}$ content $\left(c_{C}\right)$ have different $p_{\mathrm{CO} 2}(t)$ trajectories (different colors of the same thickness in Figure 9). This is because, for the same total amount of $\mathrm{C}$, the fraction of $\mathrm{C}$ stored in the ocean differs between cation cases. However, the trajectories for different cation cases bunch together at high $c_{C}$ (thick lines of all colors in Figure 9). That is because, for high total atmosphere+ocean $\mathrm{C}$ inventories, the ocean is saturated and most of the $\mathrm{C}$ is in the atmosphere. When $(\mathrm{C}$ in atmosphere $) \gg(\mathrm{C}$ in ocean $), \mathrm{C}_{\mathrm{atm}} \approx \mathrm{C}_{\text {total }}$, independent of how much $\mathrm{C}$ is in the ocean. The colors also bunch together for very low $c_{C}$. For such worlds, the $p_{\mathrm{CO} 2}$ is too low to affect climate until $T_{\text {surf }}>100{ }^{\circ} \mathrm{C}$. As a result, such worlds have $\mathrm{H}_{2} \mathrm{O}$ as the only greenhouse gas. Then, very low $c_{C}$ worlds deglaciate at the same time (for a given planet orbital semimajor axis), trek quickly across the narrow "neck" at the bottom of the diagram on Figure 2, and then suffer the runaway greenhouse.

In Figure 8, cases with high $\mathrm{CO}_{2}$-equivalent atmosphere+ocean $\mathrm{C}$ content have longer durations with habitable surface water $\left(\tau_{h a b}\right)$. The longest-lived worlds maintain $p_{\mathrm{CO} 2}$ in the $0.2-20$ bar range for Gyr (Figure 9.).

Worlds with planet water mass fraction $=0.1$ (Figure 10 show greater persistence of HP-ice. This is because $f_{W}=0.1$ seafloor pressure is $12 \mathrm{GPa}$ (for $M_{p l}=1 M_{\oplus}$ ), and these high pressures favor HP-ice (Figure 3. For a given $\mathrm{CO}_{2}$-equivalent atmosphere+ocean $\mathrm{C}$ content, the climate is colder, because the bigger ocean 


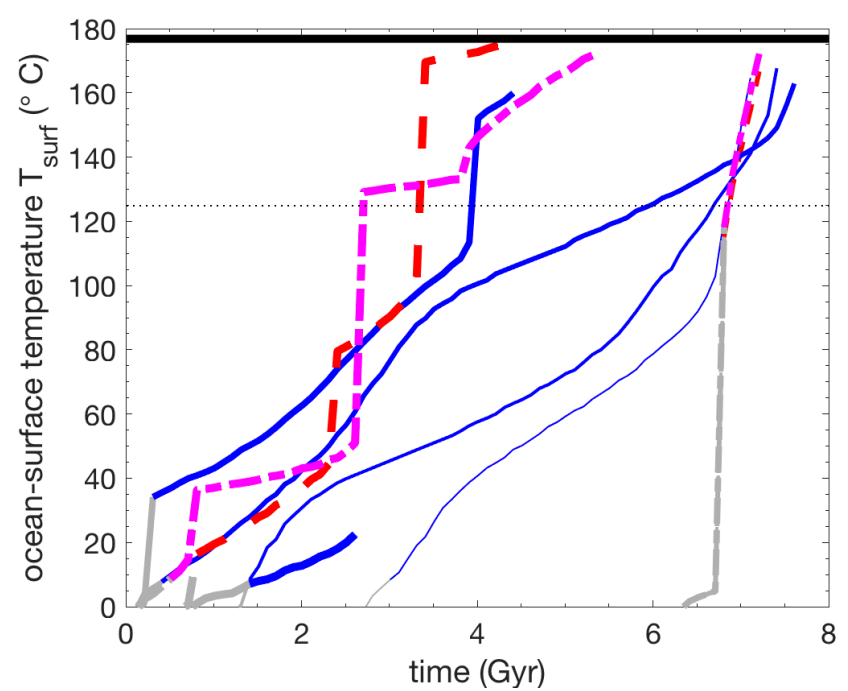

Figure 8. How ocean-surface temperature $\left(T_{\text {surf }}\right)$ evolves with time for $a=1.1 \mathrm{AU}$, planet water mass fraction $=0.01$, $M_{p l}=1 M_{\oplus}$. Dashed red lines correspond to oceans with $0.5 \mathrm{~mol} / \mathrm{kg} \mathrm{Na}$, dash-dotted magenta lines correspond to oceans with $0.25 \mathrm{~mol} / \mathrm{kg} \mathrm{Ca}$, and solid blue lines correspond to cationpoor oceans. Grayed-out parts of the lines correspond to parts of the time evolution for which high-pressure ice occurs at depth within the ocean. For these grayed-out parts, $T_{\text {surf }}$ will be an underestimate to the extent that HP-ice excludes $\mathrm{CO}_{2}$ from the matrix. The thickness of the line corresponds to the $\mathrm{CO}_{2-}$ equivalent atmosphere+ocean $\mathrm{C}$ content $\left(c_{C}\right)$, with thicker lines marking higher $c_{C}:\left\{3.5 \times 10^{4}, 1.1 \times 10^{5}, 3.5 \times 10^{5}, 1.1 \times 10^{6}\right.$, $\left.3.5 \times 10^{6}\right\} \mathrm{kg} \mathrm{m}^{-2} \mathrm{CO}_{2}$-equivalent $\mathrm{C}$ are considered. The dotted line marks 395K (highest temperature at which life has been observed to proliferate). The black bar marks $450 \mathrm{~K}$ (end of habitability). The lines end if $p_{\mathrm{CO} 2}>40$ bars.

dilutes the $\mathrm{CO}_{2}$. For high- $f_{w}$ worlds, because the ocean is the dominant reservoir of $\mathrm{C}$, small fractional changes in the storage of $\mathrm{C}$ in the ocean can lead to large fractional changes in $p_{\mathrm{CO} 2}$.

The initial $\mathrm{pH}$ of the habitable surface water ranges from 3 to $>11$. In our model, as atmosphere $p_{\mathrm{CO} 2}$ rises during the interval of habitable surface water, ocean pH falls (Zeebe 2012), typically by $<1 \mathrm{pH}$ unit. For $\left\{p_{\mathrm{CO} 2}, T_{\text {surf }}\right\}$ values that allow habitable surface water, $\mathrm{pH}$ is higher for the ' $0.25 \mathrm{~mol} / \mathrm{kg} \mathrm{Ca}$ ' case and highest of all for the ' $0.5 \mathrm{~mol} / \mathrm{kg} \mathrm{Na}$ ' case.

In summary, ocean chemistry is key to waterworld climate. This is because of the following effects. (1) Ocean $\mathrm{pH}$ sets the ocean-atmosphere partitioning of $\mathrm{C}$. This in turn sets the sensitivity of $p_{\mathrm{CO} 2}$ to increases in total $\mathrm{C}$ abundance (the Revelle factor). The Revelle factor corresponds to the differences in spacing between the $\mathrm{y}$-axis positions of lines of the same color (cation abundance) but different thickness $\left(c_{C}\right)$ in Figures 7 . 10. (2) Carbonate-system equilibria are $T$-dependent. This causes the upwards slope of the colored lines in Figure 7][10.

\subsection{Controls on the duration of habitable surface water $\left(\tau_{h a b}\right)$}

Figure 11 shows, for $f_{W}=0.01, M_{p l}=1 M_{\oplus}$ waterworlds, the onset time, shutoff time, and duration of habitable surface water. In each case, nonzero onset times (thin solid lines in Figure 11) correspond to

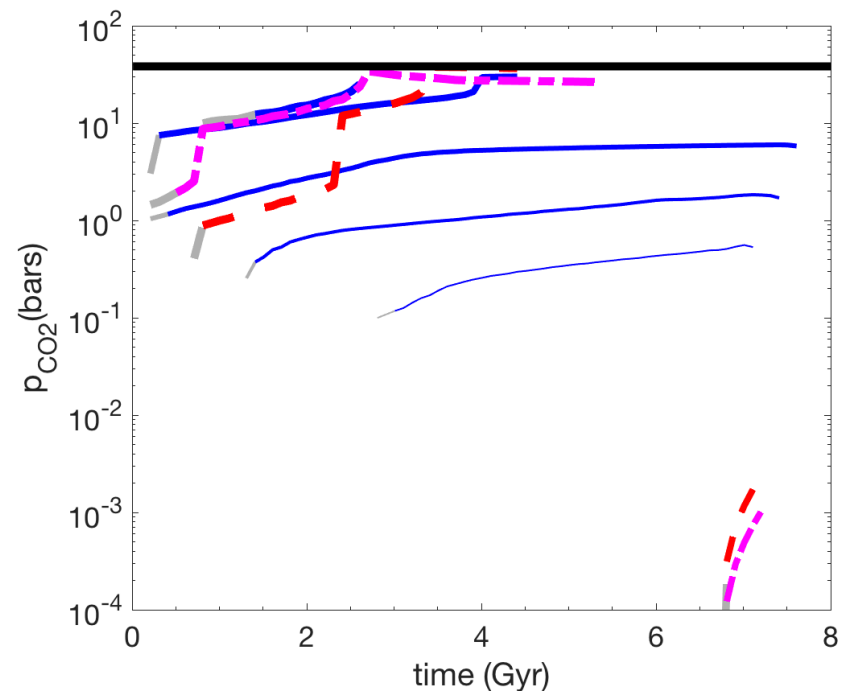

Figure 9. How $p_{\mathrm{CO} 2}$ evolves with time for $a=1.1 \mathrm{AU}$, planet water mass fraction $=0.01, M_{p l}=1 M_{\oplus}$. Dashed red lines correspond to oceans with $0.5 \mathrm{~mol} / \mathrm{kg} \mathrm{Na}$, dash-dotted magenta lines correspond to oceans with $0.25 \mathrm{~mol} / \mathrm{kg} \mathrm{Ca}$, and solid blue lines correspond to cation-poor oceans. Grayed-out parts of the line correspond to parts of the time evolution for which HP-ice occurs within the ocean. For these grayed-out parts, $T_{\text {surf }}$ will be an underestimate to the extent that $\mathrm{HP}$-ice excludes $\mathrm{CO}_{2}$ from the matrix. The thickness of the lines correspond to the $\mathrm{CO}_{2}$-equivalent atmosphere+ocean $\mathrm{C}$ content $\left(c_{C}\right)$, with thicker lines marking higher $c_{C}$ : $\left\{3.5 \times 10^{4}, 1.1 \times 10^{5}, 3.5 \times 10^{5}, 1.1 \times 10^{6}, 3.5 \times 10^{6}\right\} \mathrm{kg} \mathrm{m}^{-2} \mathrm{CO}_{2}-$ equivalent $\mathrm{C}$ are shown for the cation-poor-ocean case. For the $0.25 \mathrm{~mol} / \mathrm{kg} \mathrm{Ca}$ and $0.5 \mathrm{~mol} / \mathrm{kg} \mathrm{Na}$ cases, $c_{C}=1.1 \times 10^{6} \mathrm{~kg} \mathrm{~m}^{-2}$ $\mathrm{CO}_{2}$-equivalent $\mathrm{C}$ plots on the left half of the figure and the smaller values of $c_{C}$ plot on top of one another as the near-vertical line around $7 \mathrm{Ga}$ (only one representative low- $c_{C}$ track for each case is shown). The black bar marks 40 bars $\mathrm{CO}_{2}$ (which we deem sufficient for the end of habitability). The lines end if $T>450 \mathrm{~K}$.

the meltback time for an early-established ice lid on the ocean. Shutoff times (thin dash-dot lines in Figure 11) correspond to either the runaway greenhouse or to excessive (>40 bar) $p_{\mathrm{CO} 2}$. With increasing $\mathrm{CO}_{2}$-equivalent atmosphere+ocean $\mathrm{C}$ content, for all panels in Figure 11 . $\tau_{h a b}$ (thick solid lines in Figure 11) rises and then falls over an interval of $\sim 10^{6} \mathrm{~kg} \mathrm{~m}^{-2}$, i.e. $O\left(10^{2}\right)$ bars of $\mathrm{CO}_{2}$-equivalent. This exceeds the $O(10)$ bar $p_{\mathrm{CO} 2}$ range for optimum habitability in Figure 2. That is because around the habitability optimum, as $\mathrm{C}$ is added to the system, most of the extra $\mathrm{C}$ is partitioned into the ocean and does not contribute to $p_{\mathrm{CO} 2}$ (Archer et al. 2009).

Too much $\mathrm{C}$ makes the planet uninhabitably hot. Figure 13. which is a simplistic cartoon of $\mathrm{C}$ partitioning, shows why. Starting with a small $\mathrm{CO}_{2}$-equivalent atmosphere+ocean $\mathrm{C}$ content, $p_{\mathrm{CO} 2}$ is initially low. Adding $\mathrm{C}$ will eventually overwhelm rock-sourced cations. The ocean then becomes acidic. Once the ocean is acidic, extra $\mathrm{C}$ spills into the atmosphere and $p_{\mathrm{CO} 2}$ becomes high. Supposing the ocean storage capacity for $\mathrm{C}$ to be fixed at $\sim 0.5 \mathrm{~mol} / \mathrm{kg} \mathrm{C}$ (in reality capacity will rise with $p_{\mathrm{CO} 2}$; Equation 1), and for $\mathrm{pH}$ low enough that all aqueous $\mathrm{C}$ is stored as $\mathrm{CO}_{2}$, then ocean $\mathrm{CO}_{2}$-equivalent content is $20 \mathrm{~g} / \mathrm{kg}$ - for a $100 \mathrm{~km}$ deep ocean, $3 \times 10^{6} \mathrm{~kg} \mathrm{~m}^{-2}$. If extra $\mathrm{C}$ goes into the atmosphere, then an additional $4 \times 10^{5} \mathrm{~kg} \mathrm{~m}^{-2}$ leads to an uninhabitably hot surface. Although this "bucket model" (over-)simplifies carbon- 


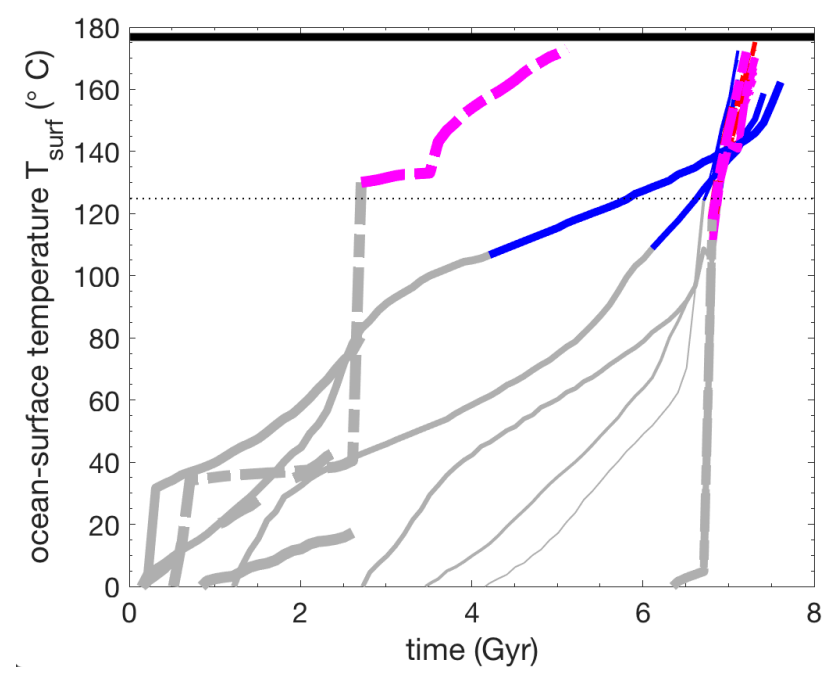

Figure 10. As Figure 8 but for planet water mass fraction $\left(f_{W}\right)=0.1$. Evolution of ocean-surface temperature $T_{\text {surf }}$, for semimajor axis $=1.1 \mathrm{AU}, M_{p l}=1 M_{\oplus}$. Dashed red lines correspond to oceans with $0.5 \mathrm{~mol} / \mathrm{kg} \mathrm{Na}$, dash-dotted magenta lines correspond to oceans with $0.25 \mathrm{~mol} / \mathrm{kg} \mathrm{Ca}$, and solid blue lines correspond to cation-poor oceans. Note greater persistence of highpressure (HP) ice (gray shrouding) at depth within the oceans. The thickness of the line corresponds to $\mathrm{CO}_{2}$-equivalent atmosphere+ocean $\mathrm{C}$ content $\left(c_{C}\right)$, with thicker lines marking higher $c_{C}$. Tracks are generally rather similar to those for $f_{W}=0.01$, except that the same climate track requires $\sim 10 \times$ more $c_{C}$ due to dilution. The dotted line marks 395K (highest temperature at which life has been observed to proliferate). The black bar marks $450 \mathrm{~K}$ (end of habitability). The lines end if $p_{\mathrm{CO} 2}>40$ bars.

ate system chemistry, it explains the $\sim 10^{6} \mathrm{~kg} \mathrm{~m}^{-2}$ upper limit for habitability for the Na-free, Ca-free case (middle panel in Figure 11). The $\mathrm{C}$ threshold for planet sterilization is raised if cations are available (left panel and right panel of Figure 11), because they neutralize the carbonic acid. For example, adding $0.5 \mathrm{~mol} / \mathrm{kg}$ of positive charges $\left(0.25 \mathrm{~mol} / \mathrm{kg}\right.$ of $\mathrm{Ca}^{2+}$, or $0.5 \mathrm{~mol} / \mathrm{kg}$ of $\left.\mathrm{Na}^{+}\right)$ means that an extra $0.5 \mathrm{~mol} / \mathrm{kg}$ of $\mathrm{C}$ must be added to get nontrivial $\mathrm{CO}_{2}$ in the atmosphere. For a $100 \mathrm{~km}$ deep ocean, this is 200 bars $=2 \times 10^{6} \mathrm{~kg} \mathrm{~m}^{-2}$. This quantity corresponds to the vertical offset between the habitability optimum in the middle panel, versus the habitability optimum in the two side panels, of Figure 11 . This quantity of $2 \times 10^{6} \mathrm{~kg} \mathrm{~m}^{-2}$ also corresponds to the vertical offset between the upper limit for habitability in the middle panel, versus the upper limit for habitability in the two side panels, of Figure 11. Neutralization by cations also explains why $\tau_{h a b}$ is brief (and uniform) for low $\mathrm{C}$ content when cation content is high. For these worlds, $p_{\mathrm{CO} 2}$ is so low (because $\mathrm{pH}$ is so high) that these planets effectively have $\mathrm{H}_{2} \mathrm{O}+\mathrm{N}_{2}$ atmospheres. Examples of evolutionary tracks for such worlds include the nearly vertical tracks to the right of Figure 8. Pure- $\mathrm{H}_{2} \mathrm{O}$ atmospheres lead to planets having habitable surface water for $<1$ Gyr if they orbit G-stars, according to the 1D model of Goldblatt (2015).

$\tau_{h a b}$ is longest for semimajor axes $a \sim 1.1$ AU. For $a<1.1$ AU habitable surface water is available from $t=0$ at the inner edge of the HZ, but the runaway greenhouse comes soon. For $a \gg 1.1 \mathrm{AU}$, the surface is frozen until near the end of the model run (and the end of the main sequence, which we decree to occur at 10 Gyr for the G-type host star, arrives swiftly). For $a \sim 1.5 \mathrm{AU}$, only a narrow range of high $\mathrm{CO}_{2}$ permits habitability, corresponding to the wing-shape extending to the top right on all panels. $\mathrm{CO}_{2}$ condensation (Turbet et al. 2017) might clip this wing of high-semimajor-axis habitability. Only around 1.1 AU does habitable surface water both begin early and end late. The 1.1 AU maximum in $\tau_{h a b}$ corresponds to the "bull's-eyes" in Figure 11 .

On a $10 \times$-deeper ocean $\left(f_{W}=0.1\right.$; Figure 12), the habitability optimum shifts to $10 \times$ larger values of $\mathrm{C}$. This shift is because more $\mathrm{C}$ is needed to overwhelm the ocean sinks (Figure 13). The range of $\mathrm{C}$ that gives long durations of surface liquid water appears narrower on a log scale. That is because the range from "minor $p_{\mathrm{CO} 2}$ to 40 bars $p_{\mathrm{CO} 2}$ " is the same $\left(\sim 4 \times 10^{5} \mathrm{~kg} \mathrm{~m}^{-2} \mathrm{C}\right.$ for fixed cations) in absolute terms, so smaller in fractional terms. The upper limit for habitability has also moved higher, but only by a factor of two. This is because [C]rich tracks that reach 40 bars $p_{\mathrm{CO} 2}$ ' at $T<100{ }^{\circ} \mathrm{C}$ always have HP ice for $f_{W}=0.1$, and so do not count as habitable (thick stubby gray lines that terminate within the bottom left of Figure 10. The strong dependence of the lifetime of habitability on semimajor axis for $f_{W}=0.01$ is more subdued for $f_{W}=0.1$. This is because almost all $f_{W}=0.1$ worlds start with HP-ice due to the deeper ocean (Figure 3), and such worlds are not counted as habitable in Figure 12 until temperature has risen sufficiently to melt all the high-pressure ice.

\subsection{Summary and results of parameter sweep}

So far we have considered three specific cationabundance cases. Now we consider a much wider range of cation abundances.

Figure 14 shows how the duration of habitable surface water $\left(\tau_{h a b}\right)$ depends on $\mathrm{CO}_{2}$-equivalent atmosphere+ocean $\mathrm{C}$ content $\left(c_{C}\right)$ and cation abundance (i.e. the extent of rock-water reaction) for semimajor axis $=1.1 \mathrm{AU}$, planet water mass fraction $=0.01$. For high $c_{C}$ (black circles in Figure 14), C swamps all available oceanic and crustal sinks, and so $\mathrm{C}$ piles up in the atmosphere (Figure 13). This leads to sterilizing surface temperatures. When cations released from rock dominate ocean chemistry, $\mathrm{pH}$ is high, and almost all $\mathrm{C}$ is sequestered as $\mathrm{CO}_{3}^{2-}$ ions, $\mathrm{HCO}_{3}^{-}$ions, or $\mathrm{CaCO}_{3}$ (Kempe \& Degens 1985; Blättler et al. 2017). Therefore little $\mathrm{C}$ remains for the atmosphere, and so $p_{\mathrm{CO} 2}$ is minimal and has little role in setting planet climate. The planet then has habitable surface water for only $<1 \mathrm{Gyr}$ (dark blue disks at the top of Figure 14). For cation-poor oceans (lower part of plot), $\tau_{h a b}$ is longest for the $[\mathrm{C}]$ which gives $p_{\mathrm{CO} 2} \sim 1$ bar at habitable temperatures. This is as expected, because the range of $L_{*}$ that permits habitable surface water is widest for $p_{\mathrm{CO} 2} \sim 1$ bar (Figure 2). Durations dwindle for lower [C] and vanish entirely for higher $[\mathrm{C}]$ (excessive $p_{\mathrm{CO} 2}$ and/or $T_{\text {surf }}$ ). When cations in the ocean balance [C] (black horizontal line), the maximum in habitable-surface-water duration occurs at larger $\mathrm{C}$. We suppose that the altered rock layer has the composition of mid-ocean ridge basalt, that it is completely leached of the charge-equivalent of its $\mathrm{Na}$ and $\mathrm{Ca}$ content, and that the leached thickness is log-uniformly distributed from $0.1 \mathrm{~km}$ to $50 \mathrm{~km}$ (Section 3.2). For the purposes of Figures $14-15$ we as- 

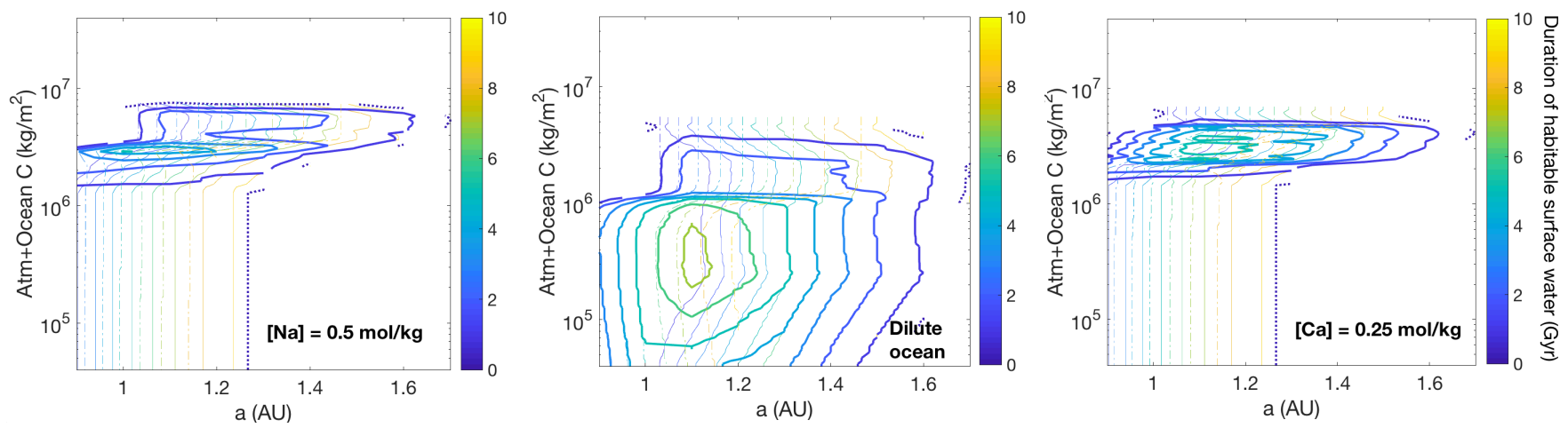

Figure 11. Waterworld $\tau_{h a b}$ (duration with habitable surface water) diagrams for planet water mass fraction $\left(f_{w}\right)=0.01, M_{p l}=1 M_{\oplus}$. Column-masses of $\mathrm{C}$ are in $\mathrm{CO}_{2}$ equivalent. Thick contours correspond to the duration with habitable surface water, in Gyr. They are spaced at intervals of $1 \mathrm{Gyr}$, except for the outer dotted contour, which corresponds to a duration of 0.2 Gyr. Thin solid contours correspond to the time of onset of habitable surface water (i.e., time after which ocean has neither HP-ice nor surface ice), spaced at intervals of 1 Gyr. Worlds to the right of the leftmost thin solid contour in each panel are "revival worlds" (worlds that start with surface ice, and subsequently deglaciate). Thin dashed contours mark the time when the planet is no longer habitable (i.e., either $p_{\mathrm{CO} 2}>40$ bars, or $T_{\text {surf }}>450 \mathrm{~K}$ ), spaced at intervals of $1 \mathrm{Gyr}$. The small wiggles in the contours, and the indentations at $\sim 3 \times 10^{7} \mathrm{~kg} \mathrm{~m}^{-2}$, are interpolation artifacts.
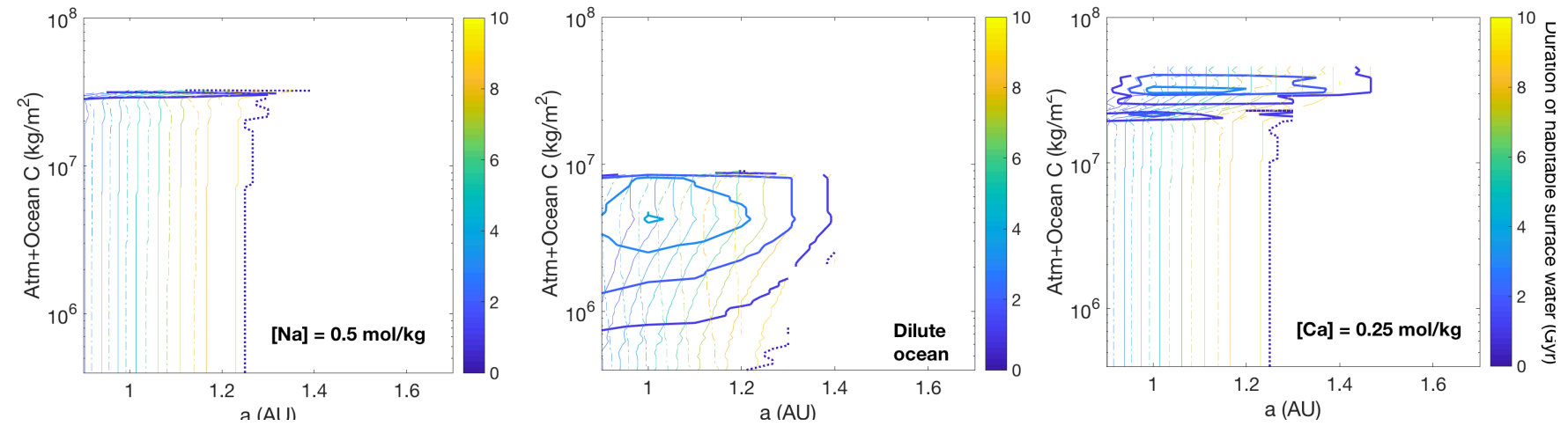

Figure 12. As Figure 11 but for planet water mass fraction $=0.1$.

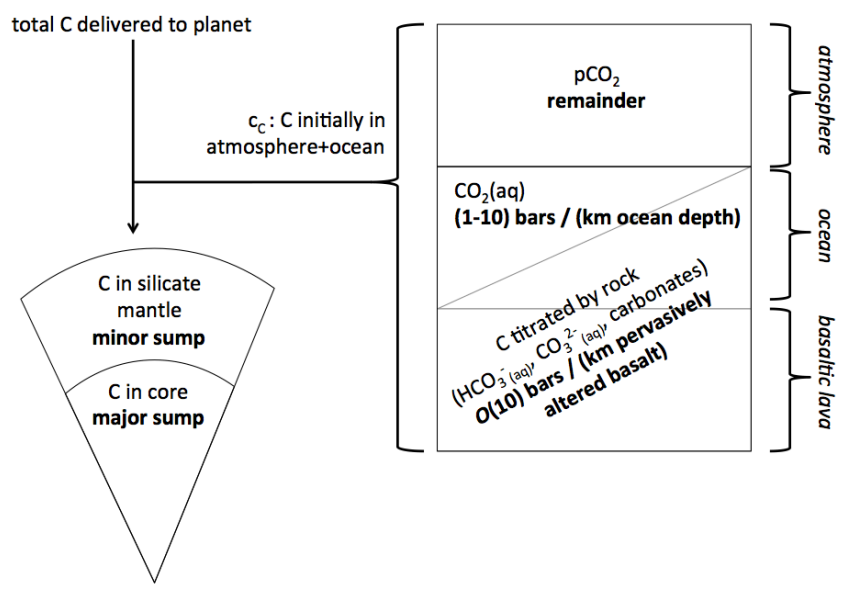

Figure 13. A simplistic "bucket" model of $p_{\mathrm{CO} 2}$.

sume that carbonates don't form; if they did form, then a factor of $\sim 2$ more $\mathrm{C}$ would be needed to neutralize the cations. Equilibrium with minerals (e.g. $\mathrm{CaCO}_{3}$, $\mathrm{NaAlSi}_{2} \mathrm{O}_{6}$ ) might buffer ocean cation content to $<0.5$ $\mathrm{mol} / \mathrm{kg}$, which roughly corresponds to the middle red line in Figure 14. Most points above this line have short $\tau_{h a b}$. Therefore, our decision to allow cation contents $>0.5 \mathrm{~mol} / \mathrm{kg}$ is geochemically less realistic, but conser- vative for the purpose of estimating $\tau_{h a b}$ on waterworlds.

To explore habitable-surface-water duration, we do a parameter sweep. We consider a hypothetical ensemble of $M_{p l}=M_{\oplus}$, planet water mass fraction $=0.01$, semimajor axis $=1.1 \mathrm{AU}$ worlds (Figure 15). Suppose that $\log \left(c_{C}\right)$ is uniformly distributed (independent of the leached thickness) from -5 to -3 $(0.3 \%-30 \%$ of water content; $\mathrm{C} / \mathrm{H}>1$ is cosmochemically implausible) (Section 3.2). This prior likely overweights small values of $c_{C}$, because for small values of non-core planet $\mathrm{C}$ mass fraction $\left(c_{C}\right), \mathrm{C}$ can be stored mainly in the silicate mantle (Hirschmann 2016). For these cosmochemically and geophysically reasonable priors, the duration of habitable surface water is shown in Figure 15. The lower panel shows that $\mathrm{C}$ has a negative or neutral effect on $\tau_{h a b}$ for slightly more than half of the parameter combinations tested. About a quarter of worlds never have habitable surface water, due to excessive initial $p_{\mathrm{CO} 2}$. Another quarter have $p_{\mathrm{CO} 2}$ so low, due to efficient rock leaching, high-pH oceans, and storage of $\mathrm{C}$ as $\mathrm{CO}_{3}^{2-}$ ion or $\mathrm{HCO}_{3}^{-}$ion, that $\mathrm{CO}_{2}$ has minor climate effect. As a result, the (short) duration of habitable surface water is about the same as for an $\mathrm{N}_{2}+\mathrm{H}_{2} \mathrm{O}$ atmosphere, and $<1$ Gyr. The remaining half of worlds have longer $\tau_{h a b}$ due to $\mathrm{CO}_{2}$. In $\sim 9 \%$ of cases, the waterworlds stay habitable for longer than the age of the Earth.

Figure 15 also shows the results for planet water mass 


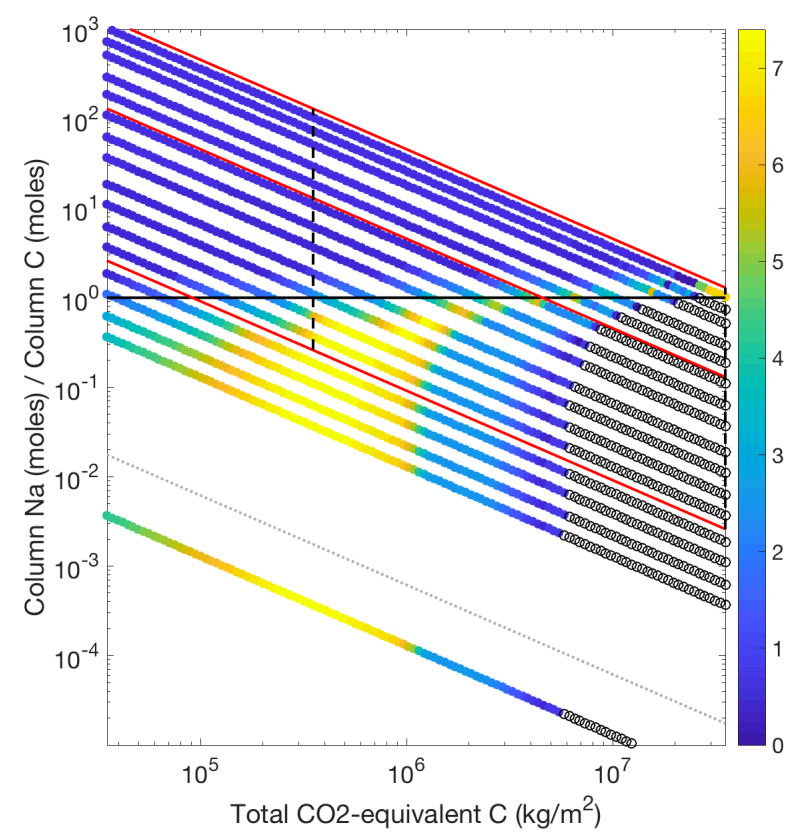

Figure 14. To show controls on the duration of habitable surface water $\left(\tau_{h a b}\right)$ on waterworlds. Color scale corresponds to $\tau_{h a b}(\mathrm{Gyr})$ for $a=1.1 \mathrm{AU}, f_{W}=0.01, M=1 M_{\oplus}$. The black circles are neverhabitable worlds. Red lines show the cation content $(\mathrm{mEq} / \mathrm{kg})$ for complete leaching of both $\mathrm{Na}$ and $\mathrm{Ca}$ - with no secondary-mineral formation - from a basalt column $50 \mathrm{~km}$ thick (top line), $5 \mathrm{~km}$ thick (middle line), or $0.1 \mathrm{~km}$ thick (bottom line). The top and bottom red lines are the bounds for cation content used in the Figure 15 parameter sweep. The vertical dashed lines at $3.5 \times 10^{5} \mathrm{~kg} / \mathrm{m}^{2}$ and $3.5 \times 10^{7} \mathrm{~kg} / \mathrm{m}^{2}$ are the bounds for $\mathrm{C}$ abundance used in the Figure 15 parameter sweep. The dotted grey line is the Na from condensation of a 100-bar steam atmosphere, assuming a volume mixing ratio in that steam atmosphere of $0.002 \mathrm{NaCl}_{(\mathrm{g})}$ (Lupu et al. 2014).

fraction $=0.1$. Durations of habitable surface water are shorter, because HP-ice can only be avoided for a narrow (steamy) range of temperatures. Reducing the maximum temperature for life from $450 \mathrm{~K}$ to $400 \mathrm{~K}$ would reduce $\tau_{h a b}$ to $<1$ Gyr on $f_{W}=0.1$ worlds. For deeper oceans, fewer worlds are sterilized by too-high $\mathrm{CO}_{2}$ values: for these worlds, the solution to pollution is dilution.

Results depend on the mean value of the ratio of $\mathrm{CO}_{2}-$ equivalent atmosphere+ocean $\mathrm{C}$ content to planet water content (i.e., $c_{C} / f_{W}$ ). If $c_{C}$ often exceeds $10^{-3}$, then a greater fraction of waterworlds will have thick $\mathrm{CO}_{2}$-rich atmospheres and be too hot for life.

Mean waterworld lifetime is $\sim 2$ Gyr (area under the curves in the lowest panel of Figure 15). This is not very much less than the maximum planet lifetime for $a=1.1 \mathrm{AU}, M_{p l}=1 M_{\oplus}$, which is 7 Gyr. After $7 \mathrm{Gyr}$, the runaway greenhouse occurs even on planets where geochemical cycles aid habitability (Caldeira \& Kasting 1992, Wolf \& Toon 2015). Therefore, cycle-independent planetary habitability is not much less effective at sustaining habitable surface water relative to a hypothetical geochemical cycle that maintains $p_{\mathrm{CO} 2}$ at the best value for habitability.

The effect of $\mathrm{C}$ on habitability in our model is positive (Figure 15, lowest panel). Our parameter sweep suggests that the median $\tau_{h a b}$ is comparable in the random-allocation-of-C case relative to a $\mathrm{C}$-starved case.
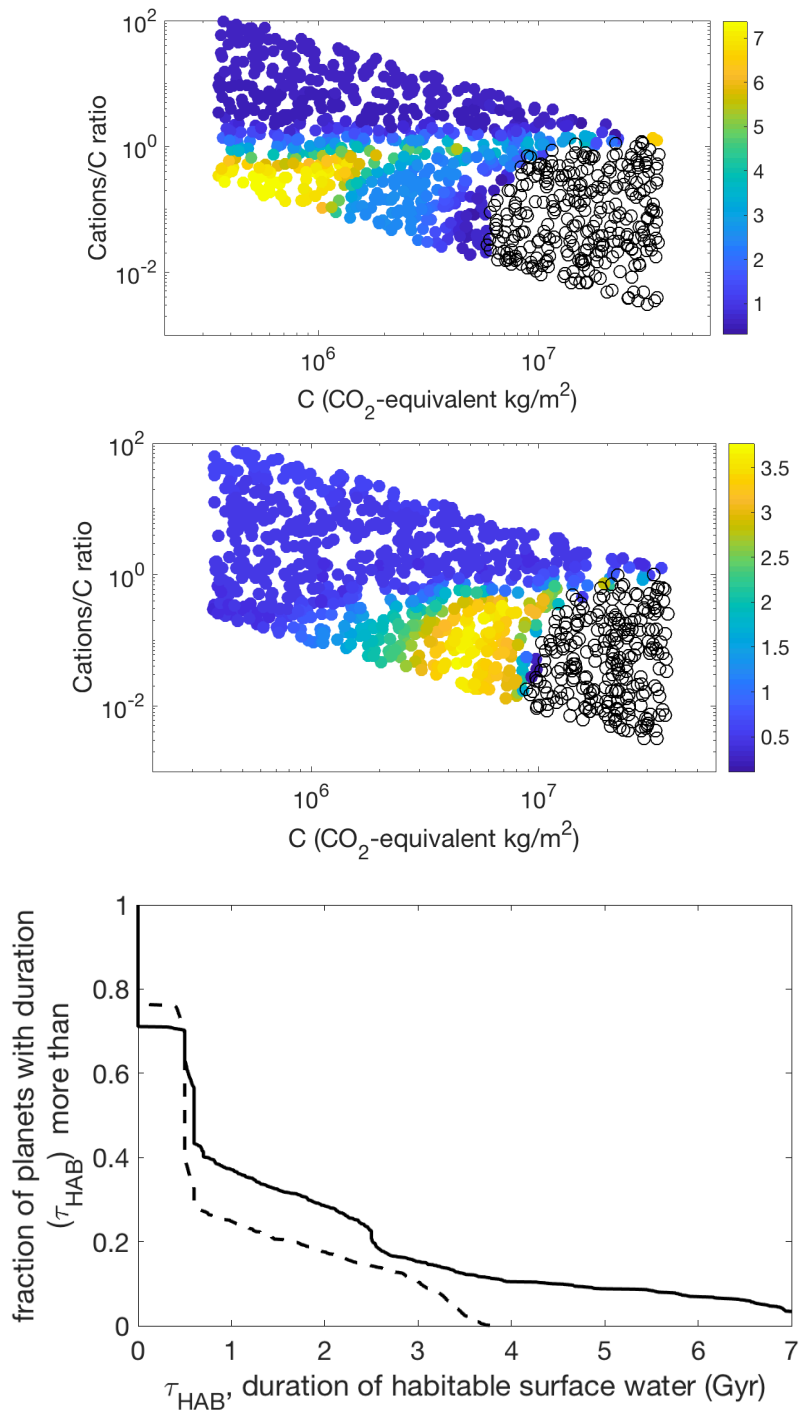

Figure 15. Duration of habitable surface water on waterworlds: results of a parameter sweep. Upper panel: the color scale corresponds to the duration of habitable surface water, $\tau_{h a b}$ (Gyr). Limits of the parameter sweep are explained in text. Open black circles are never-habitable worlds. For $M=1 M_{\oplus}$, semimajor axis $a=1.1$ AU, planet water mass fraction $\left(f_{W}\right)=0.01$. Middle panel: the same, but for $f_{W}=0.1$. Note the change in color scale. Lower panel: output sorted by the duration of habitable surface water. Solid line, $f_{W}=0.01$; dash-dot line, $f_{W}=0.1$.

Maximum $\tau_{h a b}$ is greatly increased (Carter 1983). This gain outweighs the losses due to planets that form with too much $\mathrm{C}$ for habitable surface water. Furthermore, life requires $\mathrm{C}$, so very low $[\mathrm{C}]$ in the ocean could hinder life's origin and persistence (Kah \& Riding 2007).

\section{PLANET ASSEMBLY MODEL}

We carry out $N$-body simulations (Section 5.1) in order to get a physically well-motivated ensemble of $\left\{a, M_{p l}, f_{W}\right\}$-tuples as input for the waterworld evolution code. We choose initial and boundary conditions that cause planets to migrate, as this is the easiest way to form HZ waterworlds. In Section 5.2, we will apply a volatile tracking code to the mass and orbit histories output by the $N$-body simulations. Embryos are assigned an initial volatile mass percentage that ramps from zero well inside the snowline to $f_{W, \max }$ well beyond the snowline. 
The embryos evolve via migration and mutual gravitational scattering, and develop into planets, some of which sit within the HZ. Pebbles (Sato et al. 2016, Levison et al. 2015) and planetesimals are not tracked. The volatile content of the forming planets is tracked as the embryos merge. Readers interested only in geoscience may skip this section.

\subsection{N-body model ensemble}

We perform $N$-body simulations including simple gravitational interactions with a smooth, 1D gas disk with a surface density proportional to $r^{-3 / 2}$, but not including the back-reaction of disk perturbations on the planets. This results in a simple strong-migration scenario, similar to that in many other studies (e.g. Cossou et al. 2014, Ogihara et al. 2015; Sun et al. 2017; Izidoro et al. 2017, Ronco and de Elía 2018; Raymond et al. 2018). For the purposes of this study, the $N$-body simulations serve to provide illustrative migration and collisional histories. Therefore, details of the $N$-body simulations are not essential; some readers may choose to skip to Section 5.2 .

We employ the REBOUND $N$-body code (Rein \& Liu 2012 ) and its IAS15 integrator (Rein \& Spiegel 2015), with additional user-defined forces to represent gravitational tidal drag from the disk (Zhou \& Lin 2007). Each simulation is initialized with dozens of planetary embryos near or beyond the $\mathrm{H}_{2} \mathrm{O}$ snowline, orbiting a solar-mass star. No gas giants exist at the start of our simulation, consistent with the wetter-than-solar-system cases we model (Batygin \& Laughlin 2015, Morbidelli et al. 2016). The following parameters are varied across simulations: the total mass in embryos (from 10 to $40 M_{\oplus}$ ); the outer edge of the disk (from 3 to $10 \mathrm{AU}$ ); and a scale factor for the mass of the gas disk relative to a fiducial minimum mass solar nebula (from 2-to-16 times more massive than the minimum mass solar nebula).

For each set of model parameters, we generate multiple sets of initial conditions, resulting in a total of $>145 N$-body runs. Embryo initial masses are $\sim 1$ Mars mass. The initial semi-major axes are drawn from a power-law distribution with a power-law index of -1.5. Orbits are initialized with modest eccentricities and low inclinations (relative to the plane of the gas disk). The initial orbital eccentricities and inclinations are drawn from Rayleigh distributions with a scale parameter of 0.1 for eccentricity, and 0.1 radians for inclination. In practice, the eccentricities and inclinations within a system rapidly damp to a level where damping is balanced by $N$-body excitation from neighbors. This choice of initial conditions helps to avoid a very brief phase of rapid collisions before the system is able to relax to a physically plausible set of inclinations and eccentricities. The three remaining angles (argument of pericenter, longitude of ascending node, mean anomaly) were drawn from uniform distributions $(0 \leftrightarrow 2 \pi)$.

While the gas disk is present, orbital migration is parameterized following Eqns. 19 and 20 from Zhou \& Lin (2007). The gravitational accelerations due to tides from the gas disk are proportional to: (a) the difference in the velocity of the planet (or embryo) from the circular velocity at the planet's current position (i.e., proxy for the gas velocity), (b) the planet-star mass ratio, (c) a $1 / a^{2}$ term

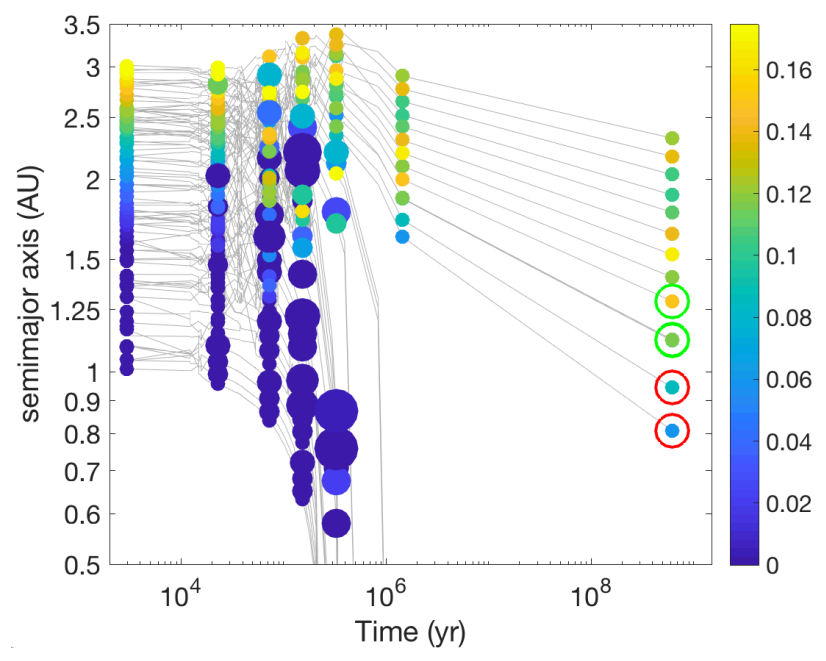

Figure 16. Example output from our planet assembly model, showing stages in planet growth. The color scale shows $\mathrm{H}_{2} \mathrm{O}$ fraction $\left(f_{W}\right)$. Red rings mark planets that initially have habitable surface water. Green rings mark HZ planets with initially frozen surfaces. Disk size corresponds to planet mass. Planets migrate inwards for the first few Myr of the simulation subject to parameterized, imposed migration torques.

that accounts for a $a^{-3 / 2}$ power-law dependence of the gas-disk surface density, and (d) a time-dependent factor that exhibits first slow dispersal, and then rapid dispersal due to photoevaporation. This prescription results in orbital migration, eccentricity damping, and inclination damping that have self-consistent timescales (Zhou \& Lin 2007). For each of our simulations, the gas-disk mass initially undergoes exponential decay with a characteristic timescale of 2 Myr. Starting at 3 Myr, the gas-disk decay accelerates due to photoevaporation, so the exponential decay rate is decreased to 50,000 years. After $4 \mathrm{Myr}$, the gas-disk mass has essentially dispersed, so the gas-disk mass is set to zero and the simulation becomes a pure $N$-body integration.

Following gas disk dispersal, planets continue to scatter and collide with one another. We consider planets as having collided when they come within a radius corresponding to a density of $0.125 \times$ that of Mars. Collisions are assumed to be perfect mergers in the $N$-body code.

Of our simulations, $29 \%$ of the runs resulted in one or more planets in the HZ, based on the zero-age main sequence luminosity of the Sun $(\sim 0.7 \times$ present solar luminosity). We set aside $N$-body simulations where no planets survived exterior to the $\mathrm{HZ}$, as the results of those simulations may be impacted by edge effects (i.e., if embryos formed beyond the outer end of our initial set of embryos). In some cases, multiple planets in the $\mathrm{HZ}$ at the end of the simulation have semimajor axes differing by less than $<0.05 \mathrm{AU}$ from one another. In these cases, we merge such planets, as they are expected to collide if we were able to extend each of the $N$-body simulations. We do not include the loss of water due to these (final) giant impacts. After applying these filters, we are left with $\sim 30 \mathrm{HZ}$ planets.

\subsection{Volatile-tracking model}

Embryo $\mathrm{H}_{2} \mathrm{O}$ content is set as follows. We represent the cumulative effects of the evolving $\mathrm{H}_{2} \mathrm{O}$-ice snowline on the water mass fraction of planetary embryos $f_{W, o}$ 
(Hartmann et al. 2017, Piso et al. 2015) by

$$
\begin{aligned}
& f_{W, o}=f_{W, \max }\left(a-a_{s l} / a_{s l}\right), a_{s l}<a<2 a_{s l} \\
& f_{W, o}=f_{W, \max }, a>2 a_{s l} \\
& f_{W, o}=0, a<a_{s l}
\end{aligned}
$$

where $a_{s l}=1.6 \mathrm{AU}$ is an anchor for the evolving snowline (Mulders et al. 2015). For each $N$-body simulation, we consider $f_{W, \max }=\{50 \%, 20 \%, 5 \%, 1 \%\}$. The highest value, $50 \%$, corresponds to theoretical expectations from condensation of solar-composition gas (Ciesla et al. 2015). Intermediate values match density data for Ceres ('Thomas et al. 2005) and inferences based on Earth's oxidation state (Rubie et al. 2015, Monteux et al. 2018). $5 \%$ corresponds to meteorite (carbonaceous chondrite) data. Why are the carbonaceous chondrites relatively dry? One explanation involves heating of planetesimals by short-lived radionuclides (SLRs; ${ }^{26} \mathrm{Al}$ and ${ }^{60} \mathrm{Fe}$ ), leading to melting of water ice. Melt-water then oxidizes the rocks, and $\mathrm{H}_{2}$ returns to the nebula (e.g., Young 2001; Rosenberg et al. 2001, Monteux et al. 2018). Because most protoplanetary disks probably had fewer SLRs than our own Gaidos et al. 2009, Gounelle 2015, Lichtenberg et al. 2016; Dwardakas et al. 2017; see also Jura et al. 2013), this process would operate differently (or not at all) in other planetary systems. Another explanation for the dryness of carbonaceous chondrites relies on Jupiter's early formation (Morbidelli et al. 2016); gas giants of Jupiter's size and semimajor axis are not typical. The range of $f_{W, \max }$ we consider corresponds to processes occurring during growth from gas+dust to embryos. We neglect $\mathrm{H}_{2} \mathrm{O}$ loss by XUV-stripping at the pre-embryo stage (Odert et al. 2018).

Growing planets shed water during giant impacts. For volatile-rich planets, at the embryo stage and larger, the most plausible mechanism for losing many wt\% of water is giant impacts. The shift to giant-impact dominance can be seen by comparing Figures 14-15 of Schlichting et al. 2015, and extrapolating to the smallest volatile layer considered in this paper $\left(10^{8} \mathrm{~kg} \mathrm{~m}^{-2}\right)$. Such deep volatile layers greatly inhibit volatile loss by $r<50 \mathrm{~km}$ projectiles, and blunt the escape-to-space efficiency of larger projectiles. (For volatile-poor planets, by contrast, small impacts are extremely erosive; Schlichting et al. 2015.) Combined with the expectation that most of the impacting mass is in the largest projectiles, erosion by giant impact is the main water loss process for waterworlds.

$\mathrm{H}_{2} \mathrm{O}$ is attrited by individually-tracked giant impacts (Marcus et al. 2010a; Stewart et al. 2014, Genda \& Abe 2005), using the relative-velocity vectors from the $N$ body code. The dependence on relative velocity of fractional ocean loss is taken from Stewart et al. (2014). Because the parameterization of Stewart et al. (2014) is for $f_{W} \sim 10^{-4}$, and ocean loss efficiency decreases with ocean thickness (Inamdar \& Schlichting 2016), this is conservative in terms of forming waterworlds. More recent calculations find lower ocean-loss values (Burger et al. 2018). When embryos collide with other embryos, or collide with planets (Golabek et al. 2018; Maindl et al. 2017), Fe-metal cores are assumed to merge efticiently. We do not track protection of $\mathrm{H}_{2} \mathrm{O}$ from giant impacts by dissolution within deep magma oceans formed during earlier giant impacts (Chachan and Stevenson 2018).

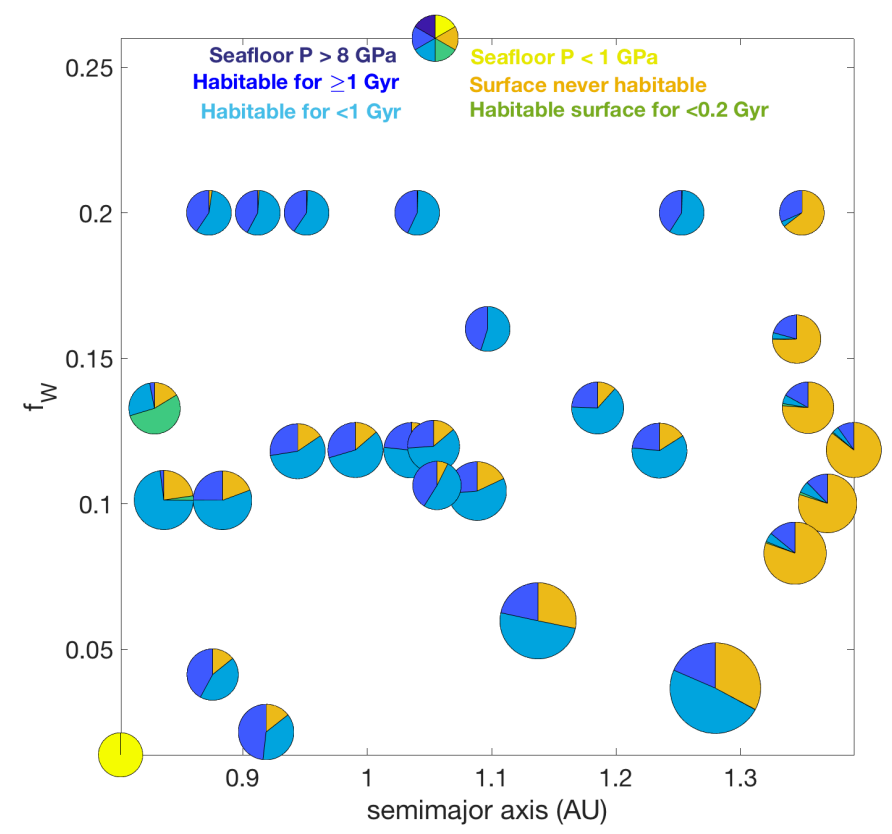

Figure 17. Fate of the $\mathrm{HZ}$ planets generated by an ensemble of $N$-body simulations. Model output for maximum embryo water content $\left(f_{W, \max }\right)=20 \%$. Disk size increases with $M_{p l}$, which ranges from 0.1 to $1.5 M_{\oplus}$. For each planet, 1000 random draws are made from a cosmochemically reasonable range of initial atmosphere+ocean $\mathrm{C}$ content $\left(c_{C}\right)$ and (independently) from a geophysically reasonable range of cation abundance (Section 3.2), and the resulting durations of habitable surface water are computed using the methods of Section 4. The disk colors summarize the outcomes. The yellow planet at $a=0.85 \mathrm{AU}$ has seafloor pressures too low to suppress $\mathrm{C}$ exchange between the convecting mantle and the water ocean. Dark blue colors correspond to $\tau_{h a b} \geq 1$ Gyr. Zero worlds have seafloor $P>8 \mathrm{GPa}$, so no worlds are colored purple. See Section 5.3 for a discussion.

This is also conservative in terms of forming waterworlds.

\subsection{Results}

Planet assembly is relatively gentle in our model. Most planet-forming collisions occur while disk gas maintains low planet eccentricities and inclinations. As a result, $\left|v_{\infty}\right|$ is small, and because giant-impact loss is sensitive to $\left|v_{\infty}\right|$, these early collisions have individually-minor effects on planet mass. When $f_{W} \geq 0.15$, an individual giant impact does not remove a large percentage of the initial planet volatile endowment (Figure 16).

Planet water mass fraction is anti-correlated with $M_{p l}$, due to the larger number of devolatilizing giant impacts needed to assemble larger planets. For a given $M_{p l}$, worlds assembled via collisions between equal-mass impactors have higher $f_{W}$ than worlds that are built up piecemeal (one embryo added a time). This is because piecemeal assembly requires a greater number of giant impacts (Inamdar \& Schlichting 2016). There is no obvious trend of $f_{W}$ with $a$ (nor $M_{p l}$ with $a$ ) within the $\mathrm{HZ}$.

The final planet water mass fractions are sensitive to $f_{W \text { max }}$, as expected (Mulders et al. 2015 Ciesla et al. 2015). For a given $f_{W, \max }$, seatloor pressures are bunched because both the weight per $\mathrm{kg}$ of water, and the number of water-removing giant impacts, increase with planet mass. None of the $f_{W, \max }=0.05$ or $f_{W, \max }=0.01$ planets have seafloor pressures $>1 \mathrm{GPa}$. By contrast, $97 \%$ of the 
$f_{W, \max }=0.2$ worlds have 1-8 GPa seafloor pressure. Finally, of the $f_{W, \max }=0.5$ worlds, $7 \%$ have seafloor pressure $<1 \mathrm{GPa}, 3 \%$ have seafloor pressure $\sim 6 \mathrm{GPa}$, and $90 \%$ have seafloor pressure $>8 \mathrm{GPa}$. These relatively low seafloor pressures are the consequence of low surface gravity: $M_{p l}$ averages $0.3 M_{\oplus}$ for this ensemble. To calculate the gravitational acceleration experienced by the ocean, we follow Valencia et al. (2007) and let $R_{\text {sea floor }} / R_{\oplus}=\left(M_{p l} / M_{\oplus}\right)^{0.27}$. If more massive planets had emerged in our simulations, then we would expect seafloor pressures to be higher for a given $f_{W, \max }$.

We injected the $\left\{a, M_{p l}, f_{W}\right\}$ tuples from the $f_{W, \max }=0.2$ planet assembly run into the waterworld evolution code, varying the $\mathrm{CO}_{2}$-equivalent atmosphere+ocean $\mathrm{C}$ content and varying cation-leaching using the same procedure and the same limits as in Section 4. The results (Figure 17) show that habitable surface water durations $>1$ Gyr occur for $\sim 20 \%$ of the simulated parameter combinations. $\tau_{h a b}>4.5$ Gyr occurs for $\sim 5 \%$ of the simulated parameter combinations. Only around a third of trials never have habitable surface water. Neverhabitable outcomes can correspond to always-icy surfaces (especially near the outer edge of the $\mathrm{HZ}$ ), or to $p_{\mathrm{CO} 2} \gtrsim$ 40 bars. Never-habitable outcomes become less likely as planet water mass fraction increases, mainly because of dilution. These results are illustrative only because mean (and median) $M_{p l}$ for this ensemble is $0.3 M_{\oplus}$. The lower gravity of $0.3 M_{\oplus}$ worlds relative to our $1 M_{\oplus}$ reference will move the inner edge of the $\mathrm{HZ}$ further out than is assumed in Figure 17 (Kopparapu et al. 2014).

\section{DISCUSSION}

\subsection{All small-radius $H Z$ planets are potentially waterworlds}

Waterworlds cannot be distinguished from bare rocks by measuring radius and density. Even if radius and density measurements were perfect, a small increase in core mass fraction could offset a $100 \mathrm{~km}$ increase in ocean depth (Rogers \& Seager 2010). Indeed, scatter in core mass fraction is expected for planets assembled by giant impacts (Marcus et al. 2010b). The best-determined planet radius is that of Kepler-93b; $1.481 \pm 0.019 R_{\oplus}$ (Ballard et al. 2014), an error of $120 \mathrm{~km}$ in radius. Yet a $120 \mathrm{~km}$ deep ocean is enough to be in the cycleindependent planetary regime discussed in this paper, and a $0 \mathrm{~km}$ deep ocean is a bare rock. Small uncertainties in star radius, or in silicate-mantle composition (e.g. Dorn et al. 2015, Stamenković \& Seager 2016) can also affect retrievals. Therefore, all small-radius HZ planets are potentially waterworlds, and this will remain the case for many years (Dorn et al. 2017; Simpson 2017, Unterborn et al. 2018).

\subsection{Climate-stabilizing feedbacks?}

The main climate feedback in our code is the decrease in $\mathrm{CO}_{2}$ solubility with increasing temperature, which is usually destabilizing (Section 4.1). We now speculate on possible feedbacks, not included in our code, that might stabilize climate.

1. When carbonates dissolve, $\mathrm{CO}_{2}$ is consumed (sic; Zeebe 2012). This is because (for circumneutral pH) one mole of $\mathrm{Ca}^{2+}$ charge-balances two moles of
$\mathrm{HCO}_{3}^{-}$, whereas $\mathrm{CaCO}_{3}$ pairs one mole of Ca with only one mole of $\mathrm{C}$ (Figure 4). Carbonates dissolve with rising $T$ (Dolejš \& Manning 2010), so the corresponding drawdown of $\mathrm{CO}_{2}$ is a climate-stabilizing feedback. This feedback is curtailed on Earth by the build-up of an lag of insoluble sediment, for example continentderived silt and clay. Insoluble sediments would be in short supply on waterworlds, and so the carbonatedissolution feedback could be stronger on waterworlds.

2. Pervasive aqueous alteration lowers the density of the upper crust. Low density of altered layers may cause rising magma to spread out beneath the altered layer, failing to reach the surface as an extrusive lava, and instead crystallizing in the subsurface as an intrusion. Intrusions have low permeability and so are resistant to alteration (in contrast to extrusive lavas that have high permeability). This negative feedback on aqueous alteration might limit cation supply.

3. When high-pressure ice forms, it excludes $\mathrm{C}$ from the crystal structure. This raises the $\mathrm{C}$ concentration in the ocean, and thus $p_{\mathrm{CO} 2}$. This negative feedback on cooling will have a strength that depends on the extent to which $\mathrm{C}$ is taken up by clathrates (Levi et al. 2017). Moreover, according to Levi et al., the solubility of $\mathrm{CO}_{2}$ in water in equilibrium with $\mathrm{CO}_{2}$ clathrate decreases with decreasing temperature. This solubility behavior is an additional negative feedback.

4. High $\mathrm{C}$ concentration lowers $\mathrm{pH}$. This favors rock leaching, which is a negative feedback on $p_{\mathrm{CO} 2}$ (Schoonen \& Smirnov 2016). But because water/rock ratio is effectively a free parameter over many orders of magnitude, the effect of water/rock mass ratio is more important in our model (Figure 18). Moreover, high-pH $\mathrm{NaOH}$ solutions corrode silicate glass, a positive feedback.

5. It has been suggested that carbonate in seafloor basalt is an important $\mathrm{C}$ sink on Earth, and has a temperature-dependent formation rate, and so might contribute to Earth's climate stability (Coogan et al. 2016).

These feedbacks might extend $\tau_{h a b}$ beyond the alreadylong durations calculated in Section 4.

\subsection{Cycle-dependent versus cycle-independent planetary habitability}

The number of planet-years of habitable surface water enabled by cycle-independent planetary habitability on waterworlds $\left(T_{\text {nocycle }, w w}\right)$ can be estimated as follows:

$$
T_{\text {nocycle }, w w}=\sum_{i=1}^{\eta_{\oplus} N_{*}} \tau_{h a b, i}\left(f_{W, i}, c_{C, i}, M_{p l, i}, \ldots\right)
$$

where $N_{*}$ is the number of Sun-like stars in the galaxy, and $\eta_{\oplus}(\approx 0.1)$ is the number of HZ small-radius exoplanets per Sun-like star. Given a $\tau_{h a b}$ look-up table (e.g. Figure 15), Equation 6 can be evaluated (e.g. Figure 17) given estimates (from plate formation models constrained by data) of the distribution of $f_{W}, c_{C}, M_{p l}$, and so on. How does $T_{\text {nocycle,ww }}$ compare to the number 
of planet-years of habitable surface water maintained by geochemical cycles $\left(T_{\text {cycle }}\right)$ ? We have

$$
\frac{T_{\text {cycle }}}{N_{*} \eta_{\oplus}} \sim x_{I C} x_{\text {evo }} 5 \mathrm{Gyr}
$$

Here, $x_{I C}$ is the fraction of small-radius HZ exoplanets with suitable initial conditions for habitability maintained by geochemical cycles, $x_{\text {evo }}$ accounts for planets where geochemical cycles initially maintain habitability but subsequently fail to do so, and 5 Gyr is a typical maximum duration of habitable surface water (before the runaway greenhouse) for a planet initialized at random $\log (a)$ within the HZ of a Sun-like star (Figure 2).

Despite the familiarity of habitability maintained by geochemical cycles, it is possible that $T_{\text {cycle }} \ll T_{\text {nocycle,ww }}$. This is because $x_{I C}$ and $x_{\text {evo }}$ are both very uncertain. This uncertainty is because predicting geochemical cycles involving habitable environments using basic models is difficult. This difficulty can be traced to the low temperatures of habitable environments. These low temperatures bring kinetic factors - such as grain size, permeability, catalysts, and rock exposure mechanisms - to the fore (White \& Brantley 1995). It is also difficult to forensically reconstruct the processes that buffer Earth's $p_{\mathrm{CO} 2}$ (Broecker \& Sanyal 1998; Beerling 2008). Negative feedbacks involving geochemical cycles probably buffer the post-0.4 Ga atmospheric partial pressure of $\mathrm{CO}_{2}\left(p_{\mathrm{CO} 2}\right)$ in Earth's atmosphere (e.g., Zeebe \& Caldeira 2008 Kump et al. 2000; Stolper et al. 2016). The effectiveness and underpinnings of these feedbacks on Earth are poorly understood (Edmond \& Huh 2003; Maher \& Chamberlain 2014, Galy et al. 2015; Krissansen-Totton \& Catling 2017). Therefore, it is hard to say how often these feedbacks break down or are overwhelmed due to the absence of land, supply limitation / sluggish tectonics, inhibition of carbonate formation due to low $\mathrm{pH}$ or to $\mathrm{SO}_{2}$, contingencies of tectonic or biological evolution, and so on (Abbot et al. 2012, Kopp et al. 2005, Edwards \& Ehlmann 2015; Flament et al. 2016; Bullock \& Moore 2007; Foley 2015; Foley \& Smye 2018; Halevy \& Schrag |2009, Genda 2016, Galbraith \& Eggleston 2017). Without a mechanistic understanding of planet-scale climate-stabilizing feedbacks on Earth, we cannot say whether these feedbacks are common among planets, or very uncommon (Lacki 2016).

\subsection{Limits to life}

Is $450 \mathrm{~K}$ a reasonable upper $T$ limit for habitability? A temperature of $450 \mathrm{~K}$ exceeds the highest temperature for which life has been observed to proliferate (395K, Takai et al. 2008). However, no fundamental barriers have been identified to life at $>400 \mathrm{~K}$ (COEL 2007). Theory tentatively suggests $423-453 \mathrm{~K}$ as the upper $T$ limit for life in general (Bains et al. 2015). If we lower the limit for life to $400 \mathrm{~K}$, then our model worlds with planet water mass fraction $\left(f_{W}\right)=0.1$ will almost always have HP-ice at times when their surface temperature is habitable (Figure 10. It is conceivable that Earth's $T_{\text {surf }}$ was $>350 \mathrm{~K}$ at $>3 \mathrm{Ga}$ based on isotopic data (e.g. Tartese et al. 2017 but see also Blake et al. 2010) and on the $T$ tolerance of resurrected ancestral proteins (Akanuma et al. 2013. Garcia et al. 2017).
Is 40 bars a reasonable upper $p_{\mathrm{CO} 2}$ limit for habitability? We use this upper limit mainly because, above this value, fine-tuning of $L_{*}$ is increasingly required to avoid uninhabitably high $T_{\text {surf }}$ (Figures 2 and 19). Therefore, 40 bars is a conservatively low upper limit. Above 70 bars and $300 \mathrm{~K}, \mathrm{CO}_{2}$ is a supercritical fluid. Supercritical $\mathrm{CO}_{2}$ is an excellent organic solvent and bactericidal agent. However, within the ocean, life might persist. We consider near-sea-surface habitability in this paper, but note that life proliferates at $1 \mathrm{GPa}$ (Sharma et al. 2002).

Extreme pH values destabilize simple biological molecules, but life on Earth solves these problems (COEL 2007). The $\mathrm{pH}$ requirements for the origin of life might be more restrictive.

Life as we know it requires nutrients other than $\mathrm{C}$, e.g. P. Yet it is unclear to what extent life requires resupply of such nutrients (Moore et al. 2017). To the contrary, given an initial nutrient budget and a sustained source of photosynthetic energy, a biosphere might be sustained by heterotrophy, i.e. biomass recycling. Biomass recycling is easier for waterworlds that lack land and lack ocean-mantle geochemical cycling, because biomass cannot be buried by siliciclastic sediment, nor subducted by tectonics. If biomass recycling is incomplete (Rothman \& Forney 2007), then to stave off productivity decline will require nutrient resupply. Nutrient resupply will be slower (relative to Earth) on waterworlds that lack ocean-mantle geochemical cycling, because land is absent (so sub-aerial weathering cannot occur), and volcanism is absent or minor. Moreover, the larger water volume will dilute nutrients. In our model, nutrients are supplied by water-rock reactions at the seafloor early in planet history, with limited nutrient resupply thereafter (by slow diffusive seafloor leaching, or minor ongoing volcanism). Resupply might be shut down entirely by highpressure (HP) ice. However, if HP-ice both convects and is melted at its base (as on Ganymede?), then nutrient supply to the ocean may continue (e.g., Kalousová et al. 2018; Kalousová \& Sotin 2018). Alternatively, nutrients can be supplied to habitable surface water by bolides and interplanetary dust, just as Earth's seafloor is nourished by occasional whale-falls. On a planet with habitable surface water, chemosynthesis (which requires an ongoing supply of rock) is greatly exceeded as a potential source of energy by photosynthesis. If photosynthesis occurs, then (given the likelihood of nutrient recycling), it has not clear how potentially observable parameters would scale with geological nutrient flux. Therefore, it is hard to make a testable prediction about the nutrient budgets of water-rich exoplanets that have habitable surface water.

Low-temperature hydrothermal vents, which are implied by our models, are a frequently proposed site for the origin of life (e.g. Russell et al. 2010).

\subsection{Uncertainties}

Astrophysics. It would be difficult to gather enough water to form a waterworld if habitable-zone planets form without a major contribution of material from beyond the nebula snowline.

If Fe-metal and atmophiles do not mix during giant impacts, then it is difficult to trap $\mathrm{C}$ in the core (Rubie et al. 2015). This would lead to an atmosphere+ocean that inherits the $\mathrm{C} / \mathrm{H}$ anticipated for nebular materials, 
which is more C-rich than considered here. The reaction $\mathrm{Fe}^{0}+\mathrm{H}_{2} \mathrm{O} \rightarrow \mathrm{Fe}^{2+} \mathrm{O}+\mathrm{H}_{2}$, with subsequent escape-tospace of $\mathrm{H}$, could also raise $\mathrm{C} / \mathrm{H}$.

Our results are sensitive to the assumption that initially-ice-coated worlds gather enough meteoritic debris during the late stages of planet accretion to lower albedo. If we are wrong and albedos of initially-icecoated worlds all stay so high that surface ice does not melt, then the only habitable waterworlds will be those that are unfrozen for zero-age main sequence stellar luminosity (i.e., $a \lesssim 1.1 \mathrm{AU}$ ). For $a \lesssim 1.1 \mathrm{AU}$, the runaway greenhouse occurs relatively early in stellar main sequence evolution, so if surface ice does not melt, then most waterworlds with habitable surface water will orbit stars younger than the Sun. Because many HZ planets have $a>1.1 \mathrm{AU}$, then if surface ice does not melt that would lower the number of waterworlds with habitable surface water by a factor of $>2$.

We assume that our worlds lack $\mathrm{H}_{2}$, so it is reasonably self-consistent to assume waterworlds have $\mathrm{CO}_{2}$ (and not $\mathrm{CH}_{4}$ ) atmospheres. If some $\mathrm{H}_{2}$ remains, then it could fuel early hydrodynamic escape of $\mathrm{H}_{2} \mathrm{O}$, and potentially aid Fischer-Tropsch type synthesis of organics (Gaidos et al. 2005). We assume a fixed $\mathrm{p}_{\mathrm{N} 2}, \sim 0.8$ bar, but variation in the abundance of $\mathrm{N}$ volatiles may be important, especially if planets draw material from $a \gtrsim 10 \mathrm{AU}$ (Marounina et al. 2018: Atreya et al. 2010). $\widetilde{\mathrm{N}}_{2}$ affects $T_{\text {surf }}$ by pressure broadening, Rayleigh scattering, e.t.c. (Goldblatt et al. 2013), and $\mathrm{NH}_{3}$ lowers the freezing-point. On Earth, the air:ocean partition of $\mathrm{N}_{2}$ is $\sim 10^{3}: 1$ (Ward (2012); a very massive ocean becomes a major $\mathrm{N}_{2}$ reservoir.

We neglect tidal heating (Henning et al. 2009). There is no evidence that tidal heating exceeds radiogenic heating for any planet in the HZ of an FGK star older than 100 Myr.

Geophysics. In order to make our calculations simpler, we understate the pressure needed to completely suppress $\mathrm{C}$ exchange between the convecting mantle and the water ocean. This pressure could be closer to $3 \mathrm{GPa}$ than $1 \mathrm{GPa}$ (Noack et al. 2016).

We assume stagnant-lid tectonics. We suspect stagnant lid is the default mode of planetary tectonics, in part because stagnant-lid worlds are common in the solar system whereas plate tectonics is unique, and in part because of the difficulty of explaining plate tectonics Korenaga 2013). Also, plate tectonics might require volcanism (Sleep 2000), and volcanism is curtailed on waterworlds (Kite et al. 2009). What if plate tectonics nevertheless operates on waterworlds (Noack and Breuer 2014)? In that case, relative to stagnant lid mode the lithosphere might store less $\mathrm{C}$. That is because plate tectonics involves a thin carbonated layer that is continually subducted and decarbonated (Kelemen \& Manning 2015), whereas stagnant-lid mode slowly builds-up a potentially thick layer of thermally stable carbonated rocks (Pollack et al. 1987; Foley \& Smye 2018). For a planet with plate tectonic resurfacing (or heat-pipe tectonics), the ocean may interact with a large (time-integrated) mass of rock (Sleep \& Zahnle 2001; Valencia et al. 2018). Increased rock supply makes it more likely that ocean chemistry would reach equilibrium with minerals (Sillen 1967). If plate tectonics operated without volcanism, then the seafloor would be composed of mantle rocks. These rocks, when aqueously alterated, produce copious $\mathrm{H}_{2}$ (Klein et al. 2013). Copious $\mathrm{H}_{2}$ production would also occur if the crust extruded at the end of magma ocean crystallization was very olivine-rich (Sleep et al. 2004). The alteration of mantle rocks to form serpentine would lead to a $\mathrm{Ca}^{2+}$-rich ocean, with high $\mathrm{pH}$ (Frost and Beard 2007). In addition, $\mathrm{H}_{2}$ production would affect atmospheric composition and climate (e.g. Wordsworth \& Pierrehumbert 2013b.

Geochemistry and climate. We use results from the 1D climate model of Wordsworth \& Pierrehumbert (2013a) in this study (e.g., Figure 2), which uses HITRAN $\mathrm{H}_{2} \mathrm{O}$ absorption coefficients and a fixed relative humidity of 1.0. Subsequent work by (Ramirez et al. 2014) uses improved (HITEMP) $\mathrm{H}_{2} \mathrm{O}$ absorption coefficients and an adjustable relative humidity. The results of a sensitivity test using the Ramirez et al. (2014) work are shown in Appendix E. Recent 3D GCM results validate the trends shown in Figure 2 (e.g. Leconte et al. 2013: Wolf \& Toon 2015: Popp et al. 2016 Wolf et al. 2017; Kopparapu et al. 2017). However, the amplitudes, location, and even existence of the instabilities discussed in Section 4 may depend on the specifics of the climate model, including how relative humidity and cloud cover respond to changing $T_{\text {surf }}$ (e.g. Yang et al. 2016).

Our treatment of both carbonate formation and $\mathrm{CO}_{2}$ solubility in cation-rich fluids in Section 4 is crude and this could shift the optimum $\mathrm{CO}_{2}$-equivalent atmosphere+ocean $\mathrm{C}$ content for habitability by a factor of $\sim 2$.

We neglect $\mathrm{Cl}$. We do so because $\mathrm{Cl}$ is geochemically much less abundant than $\mathrm{Na}$ and $\mathrm{C}$ (Sleep et al. 2001, Grotzinger \& Kasting 1993 Sharp \& Draper 2013 Clay 2017). Because we neglect Cl, our model understates the acidity of low-C-content worlds. Therefore, the vertical stripe of "optimal $c_{C}$ " may be drawn at too high a value of $\mathrm{CO}_{2}$-equivalent atmosphere+ocean $\mathrm{C}$ content. The $\mathrm{H}_{2} \mathrm{O} / \mathrm{Cl}$ ratio depends on the mechanism for water loss, if any. Production of $\mathrm{H}_{2}$ on planetesimals by oxidation of $\mathrm{Fe}^{0}$ and $\mathrm{Fe}^{2+}$, followed by escape-to-space of $\mathrm{H}_{2}$, gets rid of water but not $\mathrm{Cl}$. Mechanical ejection of water to space during impacts gets rid of both.

Although basalt with an elemental composition similar to that of Earth's oceanic crust - as used in Appendix D - is ubiquitous in the solar system (Taylor \& McLennan 2009), the silicate Earth is depleted in Na relative to meteorites that are believed to represent planetary building blocks (the chondrites) (Palme \& O'Neill 2014). The chondrites are themselves depleted in Na relative to equilibrium condensation calculations. A waterworld formed from low- $T$ condensates, or formed from chondrites but without Na depletion, would have Na:Al $\sim 1$. Peralkaline magma would be common, stabilizing $\mathrm{Na}$ silicates. Nasilicates dissolve readily, releasing aqueous $\mathrm{Na}$. A Narich ocean would have high $\mathrm{pH}$ and low $p_{\mathrm{CO} 2}$. With $\mathrm{Na}$-carbonates exposed at the surface, the dwarf planet Ceres may be a local example of a Na-rich ocean world (Carrozzo et al. 2018).

Large-amplitude stochastic differences in planet composition can result from a collision (versus a near miss) with an individual planetary embryo.

Our modeled water-rock reactions, which use freshwa- 
ter as the input fluid, raise $\mathrm{pH}$ due to release of (for example) $\mathrm{Na}^{+}$and $\mathrm{Ca}^{2+}$ (Appendix D) (MacLeod et al. 1994). $\mathrm{Na}$ is released from seafloor rocks (Staudigel \& Hart 1983) in our model, consistent with experiments (Gysi \& Stefánsson 2012) and Na-leaching of preserved 3.4 Ga seafloor crust (Nakamura \& Kato 2004). Moreover, on waterworld seafloors, jadeite is the stable $\mathrm{Na}$ hosting phase, and (at least for $T>400 \mathrm{C}$ ) the jadeitealbite transition is a maximum in [Na] Galvez et al. 2016). Na release into waterworld oceans is consistent with $\mathrm{Na}$ uptake at Earth hydrothermal vents (Rosenbauer et al. 1988, Seyfried 1987), because Earth seawater is Na-rich $(0.5 \mathrm{~mol} / \mathrm{kg})$, which favors $\mathrm{Na}$ uptake in hydrothermal systems. In summary, waterworld ocean $\mathrm{Na}$ concentrations $O(0.1-1) \mathrm{mol} / \mathrm{kg}$ are geologically reasonable.

\subsection{Opportunities}

Our analysis suggests the following science opportunities for waterworld research.

- More detailed modeling of water-rock reaction throughout waterworld evolution, using newly available constraints (e.g., Pan et al. 2013).

- New measurements (via numerical and/or lab experiments) of solubilities, etc., for high-pressure, lowtemperature conditions relevant to waterworlds.

- Map out rocky-planet atmospheres and atmosphereocean equilibria in volatile-abundance space, taking into improving constraints on the galactic range of $\mathrm{H} / \mathrm{Cl}, \mathrm{H} / \mathrm{N}$, and $\mathrm{H} / \mathrm{C}$ (Bergin et al. 2015). Our results are sensitive to the mean value of the ratio $\left(c_{C} / f_{W}\right)$ of $\mathrm{CO}_{2}$-equivalent atmosphere+ocean $\mathrm{C}$ content to planet water mass fraction.

- Seek to confirm or reject hints of life proliferating at $T>400 \mathrm{~K}$ on Earth (Schrenk et al. 2003). If life at $T>400 \mathrm{~K}$ is confirmed, this would underline the habitability of worlds with planet water mass fraction $\left(f_{W}\right)=0.1$, regardless of whether or not HP-ice is sufficient to prevent habitability (Figure 10.

- Investigate the long-term stability of rock-water mixtures on waterworlds. During giant impacts, rock and water are fully miscible. On planets with $f_{W} \ll 0.01$, the mixture cools quickly, and the rock settles out, because rock is insoluble in cool water. Rock-watermixture lifetime cannot exceed the conductive cooling timescale,

$$
\tau_{\text {unmix }} \lesssim \frac{\rho c_{p, f} \Delta Z \Delta z_{b l}}{(2.32)^{2} k_{\text {cond }}} \sim 1 \mathrm{Gyr}\left(\frac{\Delta Z}{200 \mathrm{~km}}\right)^{2}
$$

where $\rho$ is characteristic fluid density $\left(\sim 1100 \mathrm{~kg} \mathrm{~m}^{-3}\right)$, $c_{p, f}$ is fluid heat capacity $\left(\sim 4 \times 10^{3} \mathrm{~J} \mathrm{~kg}^{-1} \mathrm{~K}^{-1}\right), \Delta Z$ is ocean depth, $\Delta z_{b l}$ is the thickness of the conductive boundary layer, $k_{\text {cond }}$ is characteristic fluid thermal conductivity $\left(1 \mathrm{~W} \mathrm{~m}^{-1} \mathrm{~K}^{-1}\right)$, and we have made the upper-limit assumption $\Delta Z=\Delta z_{b l}$ (Turcotte \& Schubert 2011). On worlds with large planet water mass fraction, a parcel of the dense, hot, rock+water layer is stable to rapid convective swapping with the overlying cool rock-poor aqueous fluid. Convective inhibition slows mixture cooling and allows super-adiabatic temperature gradients (persistence of hot layers at depth). Thus, an ocean at habitable temperature might be underlain not by a solid seafloor but instead by a hot rockwater mixture. The true lifetime will be set by doublediffusive convection (Radko 2013, Friedson \& Gonzales 2017, Moll et al. 2017), taking account of the exotic behavior of aqueous tluids at $P>10 \mathrm{GPa}$ (e.g., Redmer et al. 2011, Tian \& Stanley 2013), and could be much less than the Equation 8 upper limit. It is not obvious whether such a system would be more habitable or less habitable than a fluid ocean underlain by a solid crust.

- Combine eH modeling (Schaefer et al. 2016 , Wordsworth et al. 2018) with pH modeling (this study).

- Evaluate the climate feedbacks proposed in Section 6.2. Explore the bestiary of exsolution-driven climate feedbacks suggested by our simple model (Section 4, Figure 7. Kite et al. 2011). Determine if any apply to Earth history.

\section{CONCLUSION: LONG-TERM STABILITY OF HABITABLE SURFACE WATER ON EXOPLANET WATERWORLDS WITH NO NEED FOR CARBONATE-SILICATE CYCLING}

Our model of long-term climate evolution on waterworlds shows long-term stability of habitable surface water can occur without geochemical cycling. Because volcanism is curtailed by seafloor pressure on waterworlds (Kite et al.2009), the planet is stuck with the ocean mass and ocean cations that it acquires during the first $1 \%$ of its history. Afterwards, ocean-atmosphere exchange sets $\mathrm{pH}$ in the ocean and $p_{\mathrm{CO} 2}$ in the air.

Our model indicates that the key controls on the habitability of waterworlds around Sun-like stars are as follows.

1. Initial water content. As initial water content (and thus, seafloor pressure) is increased relative to that of the Earth, long-term cycling of $\mathrm{C}$ between the atmosphere and the convective mantle moves from an active regime to a subdued regime. We studied planets with 1-8 GPa seafloor pressure. Seafloor pressure on such worlds suppresses rock melting, and so $\mathrm{C}$ cycling between the atmosphere and the deep interior is subdued (Figure 5), permitting cycle-independent planetary habitability (Fig 6).

2. Cation/C ratio. Cations are supplied by crust leaching. [Cation]/C values $\lesssim 1$ are needed (in our model) for $>1$ Gyr of habitability, because a cation excess draws $\mathrm{C}$ into the ocean from the atmosphere, and worlds with little atmospheric $\mathrm{C}$ have short lifetimes (Figures 14 and 15 .

3. Initial atmosphere+ocean $\mathrm{C} / \mathrm{H}$. When $\mathrm{C} / \mathrm{H}$ is large, $\mathrm{C}$ sinks are swamped and $\mathrm{CO}_{2}$ accumulates in the atmosphere. This can lead to uninhabitably high surface temperatures (Figures 13 15). When $\mathrm{C} / \mathrm{H}$ is small, the duration of surface liquid water is $<1 \mathrm{Gyr}$. Initial atmosphere+ocean $\mathrm{C} / \mathrm{H} \approx 1 \%$ by weight is optimal in our model (corresponding to $\mathrm{CO}_{2} / \mathrm{H}_{2} \mathrm{O} \approx 0.25 \%$ by weight if all $\mathrm{C}$ is present as $\mathrm{CO}_{2}$ and all $\mathrm{H}$ is present as $\mathrm{H}_{2} \mathrm{O}$ ) (Figures 11, 15. 
Around a quarter of the parameter combinations that we simulate yield waterworlds with $>1$ Gyr of habitable surface water. We assume that initially frozen surfaces are darkened by meteoritic debris; if they maintain a high albedo, then the fraction of long-lived worlds is cut by a factor of $\sim 3$. This does not mean that in the real Galaxy, $\gtrsim 10 \%$ of waterworlds are habitable for $>1 \mathrm{Gyr}$, because many key input parameters are uncertain. Based on these uncertainties, we recommend priority areas for future research (Section 6.6).

Atmosphere-lithosphere geochemical cycling appears to be necessary for Earth's long-term habitability (Moore et al. 2017). This connection led to the idea that "[g] eologic activity is crucial for a planet's maintained surface habitability because such habitability depends on the recycling of atmospheric gases like $\mathrm{CO}_{2}$ " (Kaltenegger 2017). However, as we have shown here, such cycling is not needed for multi-Gyr habitability on rocky exoplanets with deep oceans.

Our optimistic conclusions for Sun-like stars support optimism for waterworlds that orbit M-dwarfs (Turbet et al. 2018). For Sun-like stars, stellar main-sequence evolution (timescale $\tau_{M S} \sim 10 \mathrm{Gyr}$ ) will in the end destroy habitability. But for planets that orbit M-stars, $\tau_{M S} \gg 10$ Gyr. Because volcanism-requiring (Earthlike) habitability dies with volcanism after $\lesssim 10$ Gyr (Kite et al. 2009), most of the habitable volume in the Universe (including the distant future; Loeb et al. 2016) is for cycle-independent planetary habitability. For example, the TRAPPIST-1 HZ planets (Gillon et al. 2017) are small enough and old enough (Burgasser \& Mamajek 2017) that stagnant-lid volcanism should have shut down (Kite et al. 2009). But all seven of the TRAPPIST1 worlds have densities consistent with seafloor pressures in the 1-8 GPa range studied by this paper Grimm et al. 2018). Thes.erefore, the mechanisms proposed in this paper offer more hope for habitability in the TRAPPIST-1 system than volcanism-requiring habitability. However, although Earth-radius waterworlds in the HZ of FGK stars can retain their water, it is less clear whether or not this is the case for Earth-radius waterworlds in the HZ of a M star (Luger \& Barnes 2015; Ramirez \& Kaltenegger 2014 Dong et al. 2017, 2018). Although in the absence of volcanism, nutrient supply will ultimately become diffusively limited, habitable surface water on waterworlds can persist for $\gg 10$ Gyr. For these worlds, habitability has no need for geodynamic processes, but only the steady light of the star.

We are grateful to Bruce Fegley for continuing guidance on geochemical thermodynamics. We thank Norm Sleep for a stimulating and prompt formal review, which improved the manuscript. We thank Christophe Cossou, Itay Halevy, Jim Kasting, Ravi Kumar Kopparapu, Nataly Ozak, and Ramses Ramirez, for unselfish sharing of research output. We thank Dorian Abbot, Jonathan Lunine, Nadejda Marounina, Marc Neveu, and Ramses Ramirez for commenting on a draft. We thank David Archer, Paul Asimow, Fred Ciesla, Eric Fiegelson, Jim Fuller, Eric Gaidos, Marc Hirschmann, Manasvi Lingam, Mohit Melwani Daswani, Jack Mustard, Leslie Rogers, Laura Schaefer, Hilke Schlichting, Norm Sleep, Sarah Stewart, Cayman Unterborn, and
Steve Vance, who each provided useful advice. We thank the CHIM-XPT team: Mark Reed, Nicolas Spycher, and Jim Palandri. We thank Craig Manning and Tim Lichtenberg for sharing preprints. We thank the organizers of the University of Michigan volatile origins workshop. The Center for Exoplanets and Habitable Worlds is supported by the Pennsylvania State University, the Eberly College of Science, and the Pennsylvania Space Grant Consortium. Parts of this research were conducted with Advanced CyberInfrastructure computational resources provided by The Institute for CyberScience at The Pennsylvania State University (http://ics.psu.edu), including the CyberLAMP cluster supported by NSF grant MRI-1626251. The results reported herein benefitted from collaborations and/or information exchange within NASA's Nexus for Exoplanet System Science (NExSS) research coordination network sponsored by NASA's Science Mission Directorate. This work was supported by the U.S. taxpayer, primarily via NASA grant NNX16AB44G.

Author contributions: E.S.K. conceived, designed, and carried out the study, and wrote the paper. E.S.K. wrote the volatile tracking code (Section 5.2). E.B.F's contributions were focused on the $N$-body integrations (Section 5.1).

\section{APPENDIX}

A. HOW SEAFLOOR PRESSURE SUPPRESSES C EXCHANGE BETWEEN THE CONVECTING MANTLE AND THE WATER OCEAN.

With reference to Figure 5, four effects allow seafloor pressure to suppress volcanism (and thus C exchange between the convecting mantle and the water ocean) on waterworlds. These four effects (in order of importance) are as follows. (1) The solidus $T$ increases rapidly with $P$, so increasing $P$ truncates the maximum melt fraction, eventually to zero. (2) The upwelling mantle undergoes corner flow (turns sideways), so by reducing the distance between the base of the lithosphere and the maximum $P$ for melting, increasing $P$ reduces the proportion of upwelling mantle that melts in a given time. (3) $T$ just below the lithosphere is regulated to an absolute value that is fairly insensitive to the ocean depth, due to a negative feedback between $T$-dependent mantle viscosity and the viscosity-dependent rate of convective mantle cooling (Schubert et al. 2001; Korenaga \& Karato 2008; Stevenson 2003). Therefore, a waterworld mantle is colder for a given $P$ (dashed blue lines in Figure 5) than the mantle of a shallow-ocean planet (dashed gray lines in Figure 5). This further inhibits melting. (4) For the melt fractions corresponding to track A-B-C-D, melt fraction increases super-linearly as $\left(T-T_{\text {sol }}\right)^{1.5} /\left(T_{l i q}-T_{\text {sol }}\right)^{1.5}$ (Katz et al. 2003).

Because a planetary mantle undergoing stagnant-lid convection with no volcanism cools less efficiently for a given temperature than a plate-tectonics world with volcanism, a stagnant-lid planet should adjust to a mantle potential temperature that is higher than that of a planet undergoing plate tectonics (Figure 6 in Kite et al. 2009). However, models indicate that the temperature rise should be less than the difference between $1350^{\circ} \mathrm{C}$ (modern Earth mantle potential temperature) 
and $1550^{\circ} \mathrm{C}$ (hottest ambient-mantle potential temperature known on Earth, 3.0 Gya; Herzberg et al. 2010).

We use the anhydrous batch-melting solidus of Katz et al. (2003). Plausible waterworld upper-mantle water contents are similar to that of the Earth's mantle beneath mid-ocean ridges. These rocks have an average of 0.005-0.02 wt\% water (Workman and Hart 2005: Hirschmann \& Kohlstedt 2012), and this water might depress the solidus by $50 \mathrm{~K}$.

Complete shut-down of eruptions is difficult. This is because anomalously wet parcels of mantle or anomalously hot parcels of mantle (e.g. mantle plumes) will both melt more readily. However, in the waterworld approximation, fitful and low-rate volcanism associated with anomalously wet or anomalously hot mantle cannot buffer waterworld ocean chemistry.

\section{B. HIGH-PRESSURE ICE PHASES.}

High-pressure ice appears wherever the $\mathrm{H}_{2} \mathrm{O}$ adiabat intersects the pure- $\mathrm{H}_{2} \mathrm{O}$ freezing curve. (We neglect salt effects; Vance \& Brown 2013.) To build the fluid-water adiabat, we used

$$
\frac{\partial T_{a d}}{\partial P}=\frac{\alpha(T, p) T}{\rho\left(T_{i}, p_{i}\right) c_{p, f}}
$$

where $\rho$ is obtained from Table 1 of Wiryana et al. (1998) and Table 2 of Abramson \& Brown (2004), and $\alpha$ is obtained by differencing these tables. We assumed $c_{p, f} \sim 3800 \mathrm{~J} \mathrm{~kg}^{-1} \mathrm{~K}^{-1}$. The approximation of $c_{p, f}$ as constant is justified by Myint et al. (2017). We extrapolated the tables above $6 \mathrm{GPa}$, and for $T<80^{\circ} \mathrm{C}$. Clathrates were neglected. Pure- $\mathrm{H}_{2} \mathrm{O}$ freezing curves for ices VI and VII are from IAPWS (2011). We note that Alibert (2014) did not include the adiabatic heating of liquid water. This effect becomes important for deep oceans (Figure 3).

\section{POST-MAGMA-OCEAN TIMESCALES.}

Following a giant impact, the characteristic temperature at which water first reacts with the solid crust depends on the ratio between the timescales on which the fluidenvelope cools $\left(\tau_{c}\right)$, and the timescale over which the crust forms $\left(\tau_{x}\right)$ (Zahnle et al. 2007). If $\tau_{c}<\tau_{x}$, crust rocks will first react with hot (supercritical) $\mathrm{H}_{2} \mathrm{O}$ (Cannon et al. 2017). If $\tau_{c}>\tau_{x}$, crust rocks will react with water on an adiabat connected to an sea surface that is in equilibrium with insolation (Figure 3). The timescale for cooling $\tau_{c}$ is

$$
\tau_{c}=\frac{M_{p l} f_{W} c_{p, f}(2000 \mathrm{~K}-800 \mathrm{~K})}{4 \pi R_{p l}^{2}\left(F_{K I}-0.25 L_{*}(1-\alpha)\right)}
$$

where $c_{p, f}$ is the fluid heat capacity and $F_{K I}$ is the Komabayashi-Ingersoll limit (Ingersoll 1969). This gives $\tau_{c}=10^{5}-10^{6} \mathrm{yr}$ for the fluid envelopes considered in this paper. By contrast, $\tau_{x}$, the timescale for the crust to form, is poorly constrained. $\tau_{x}$ for stagnant-lid mode on waterworlds is the timescale for the initial post-magmaocean crust to form - perhaps as long as $10^{8}$ yr $(\mathrm{Ru}-$ bin et al. 2005; |Mezger et al.| 2013, Moynier et al. 2010; Turner et al. 2001; Solomatov 2015). Also relevant is the timescale for $\mathrm{C}$ delivery by bolide impacts $\left(\tau_{d y n}\right)$.
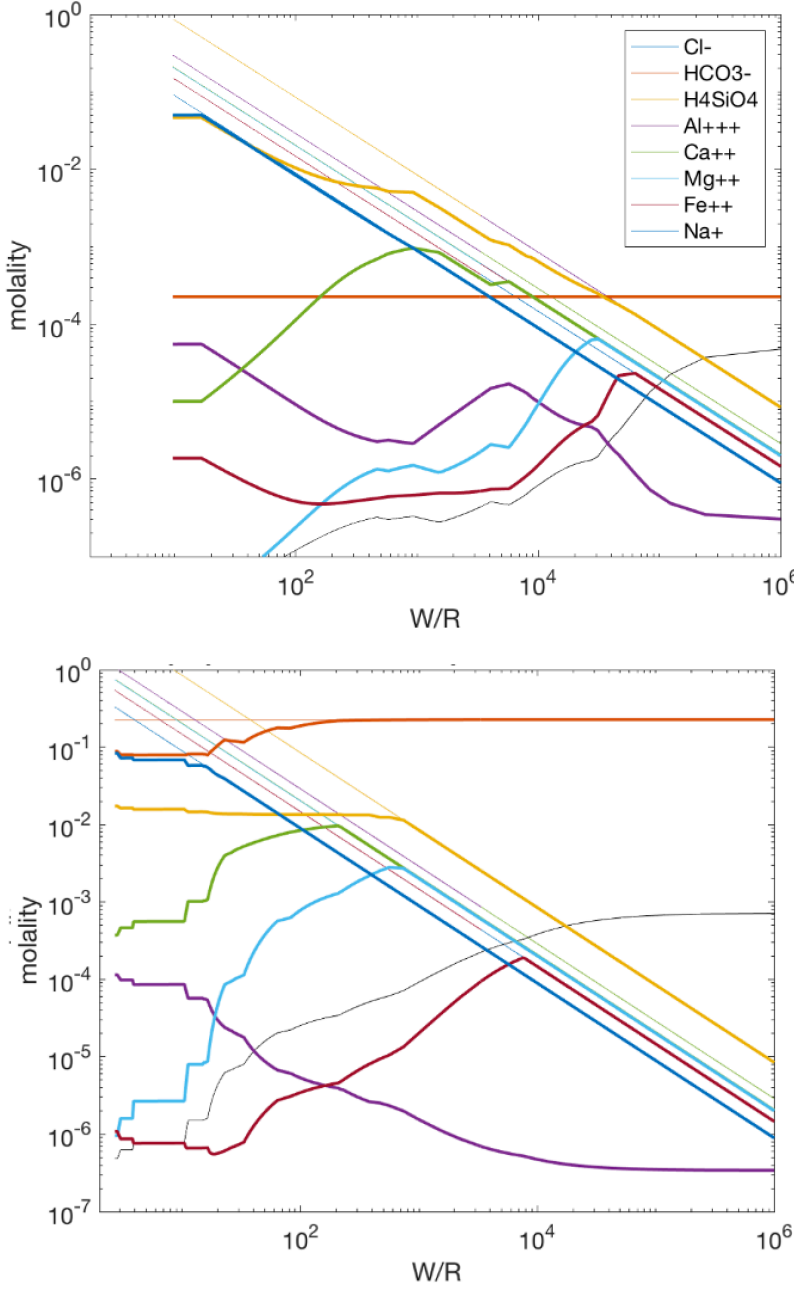

Figure 18. Seafloor basalt alteration for (upper panel) low [C] concentrations $\left(P=5000\right.$ bar, $\left.T=200^{\circ} \mathrm{C}\right)$ and (lower panel) high $[\mathrm{C}]$ concentrations $\left(P=5000 \mathrm{bar}, T=300^{\circ} \mathrm{C}\right)$. W/R is water $/$ rock ratio characterizing the reaction. The thick colored lines correspond to concentrations in the fluid. Thin lines of the same color correspond to total mol $/ \mathrm{kg}$ of rock added to the fluid. Where the total $\mathrm{mol} / \mathrm{kg}$ of rock added from the fluid deviates from the concentrations in the fluid, secondary minerals have formed. The thin black line corresponds to $-\log _{10}(\mathrm{pH})$.

$\tau_{d y n}$ could be $>10^{8} \mathrm{yr}$ for the Earth, and this may be typical of planets around G-type stars (Löhne et al. 2008). Because $\tau_{x}$ and $\tau_{d y n}$ are both plausibly much longer than $\tau_{c}$, but not plausibly shorter than $\tau_{c}$, we speculate that the characteristic temperature for basalt rock to first encounter C-rich fluid is $<650 \mathrm{~K}$. Water-rock reactions are well advanced within $10^{3}-10^{5} \mathrm{yr}$ (Hannington et al. 2005), so we expect that even $\tau_{x} \ll 1$ Myr would allow sufficient time to alter rock.

\section{MODELING OF SEAWATER-BASALT INTERACTION.}

To explore seawater-basalt reactions on waterworlds, we used CHIM-XPT v.2.4.6 (Reed 1998). We consider $T=\{298 \mathrm{~K}, 473 \mathrm{~K}, 573 \mathrm{~K}\}$. Although temperature changes over geologic time, we assume no "resetting" of seafloor alteration. "Resetting" appears to be minor for Earth seafloor alteration: seafloor basalt carbonitization occurs near the time of eruption (Coogan et al. 2016). Due to the scarcity of experiments on $\mathrm{H}_{2} \mathrm{O}$ properties for $>0.5 \mathrm{GPa}, P$ is fixed at $0.5 \mathrm{GPa}$. This low $P$ will 
cause us to understate solubilities. Improved ab initio calculations of the properties of $\mathrm{H}_{2} \mathrm{O}$ (Pan et al. 2013) will allow future improvements in waterworld seafloor modeling. We assume thermodynamic equilibrium, with solid solutions suppressed. Basalt composition is from Gale et al. (2013). A key parameter in CHIM-XPT is the water/rock mass ratio, $\mathrm{W} / \mathrm{R}$. The use of $\mathrm{W} / \mathrm{R}$ by geochemists is easy to misinterpret in an exoplanet context. For geochemists, W/R corresponds to the effective $\mathrm{W} / \mathrm{R}$ of the reactions. Reactions typically occur in zones that are not hydrologically open to the ocean. Examples are pore spaces and fractures. As a result, the reactions tend to be much more rock-influenced than the ocean as a whole. Therefore, $\mathrm{W} / \mathrm{R}$ is almost always much less than the (mass of the ocean)/(total mass of altered rock). For example, Earth hydrothermal vents have W/R of 1-100, even though the mass of pervasively water-altered rock in Earth's oceanic crust is much less than $1 \%$ of the mass of Earth's oceans. Varying W/R is a proxy for the extent of alteration of sub-oceanic crust by water. $\mathrm{W} / \mathrm{R}$ is more important than $T$ for setting vent fluid compositions.

Figure 18 shows typical results. For $\mathrm{W} / \mathrm{R}<100$ characteristic of hydrothermal reactions on Earth, outlet fluids are dominated by $\mathrm{Na}^{+}$and by dissolved $\mathrm{Si}$ species. However, dissolved Si forms solid particles upon mixing into the colder, lower-pH upper levels of the ocean (as is inferred to occur on Enceladus; Hsu et al. 2015). Therefore, we do not consider dissolved Si when calculating the sea-surface chemistry. As carbonates form, they buffer C fluid content to $\sim 0.1 \mathrm{~mol} / \mathrm{kg}$ for $\mathrm{W} / \mathrm{R} \lesssim 30$. As secondary minerals precipitate, they scrub $\mathrm{Fe}^{2+}, \mathrm{Mg}^{2+}$, and $\mathrm{Al}^{3+}$ from the fluid. Outlet fluid composition levels off at $[\mathrm{Na}] \approx 0.05-0.12 \mathrm{~mol} / \mathrm{kg}$ for $\mathrm{W} / \mathrm{R} \approx 10$ due to $\mathrm{Na}$ mineral formation, but for reasons discussed in Section 6.5 , we consider higher $[\mathrm{Na}]$ in our models.

\section{E. DETAILS OF WATERWORLD EVOLUTION CALCULATIONS.}

Here we provide more detail on the waterworld evolution calculations that are summarized in Section 3.2.

Greenhouse forcing and climate look-up table. To track waterworld climate evolution, we start from the $\operatorname{OLR}\left(p_{\mathrm{CO} 2}, T_{\text {surf }}\right)$ and albedo $\left(p_{\mathrm{CO} 2}, T_{\text {surf }}\right)$ from the $1 \mathrm{D}$ radiative-convective models of Ramirez et al. (2014) and Wordsworth \& Pierrehumbert (2013a). Here, OLR is outgoing longwave radiation $\left(\mathrm{W} / \mathrm{m}^{2}\right)$. Using the (safe) assumption that radiative equilibrium is reached so that OLR = insolation $\times(1$ - albedo $)$, we smooth and interpolate to find the $T_{\text {surf }}\left(p_{\mathrm{CO} 2}\right.$, insolation $)$ in script waterworld_surface_temperature_v3.m.

To check the sensitivity of our results to the choice of $1 \mathrm{D}$ radiative-convective climate model, we re-ran the entire waterworld evolution code substituting the output of Ramirez et al. (2014) in place of the output of Wordsworth \& Pierrehumbert (2013a). Among other advantages, the Ramirez et al. (2014) output extends to $p_{\mathrm{CO} 2}=100$ bar, avoiding the arbitrary cut-off of 40 bar in our main runs. The results are shown in Figure 19 and Figure 20. The main difference between the results is that the duration of habitable surface water for low$p_{\mathrm{CO} 2}$ planets is predicted to be shorter, and there is a greater fraction of such short-lived planets (Figure 20. However, the result that about $10 \%$ of waterworlds have

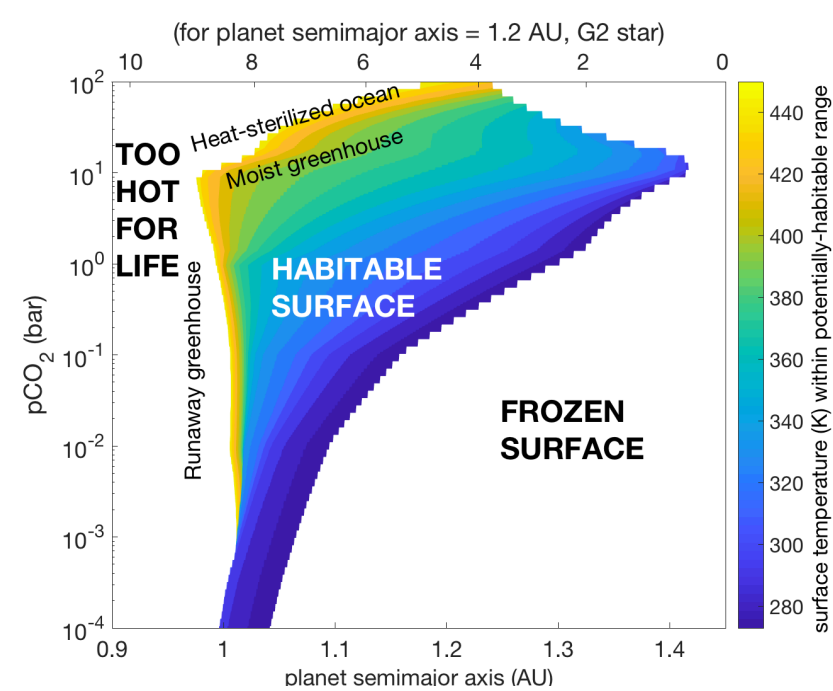

Figure 19. As Figure 2 but using the Ramirez et al. (2014) radiative-convective climate model as a sensitivity test. To show the effect of partial pressure of atmospheric $\mathrm{CO}_{2}$ and insolation $L_{*}$ on habitability, based on Ramirez et al. (2014). For G-type stars, insolation increases with age of the star on the main sequence (top axis) and decreases with distance from star (bottom axis). The colored region corresponds to the habitable zone (HZ). The planet fates define a lozenge of habitability that is broadest for $O(1)$ bar $p_{\mathrm{CO} 2}$. In other words, for a given semimajor axis, the duration of surface habitability is maximized for a "sweet spot" of $p_{\mathrm{CO} 2}$ in the range $0.2-20$ bar. The color ramp corresponds to $T_{\text {surf }}(\mathrm{K})$, and extends from $273 \mathrm{~K}$ to $450 \mathrm{~K}$.

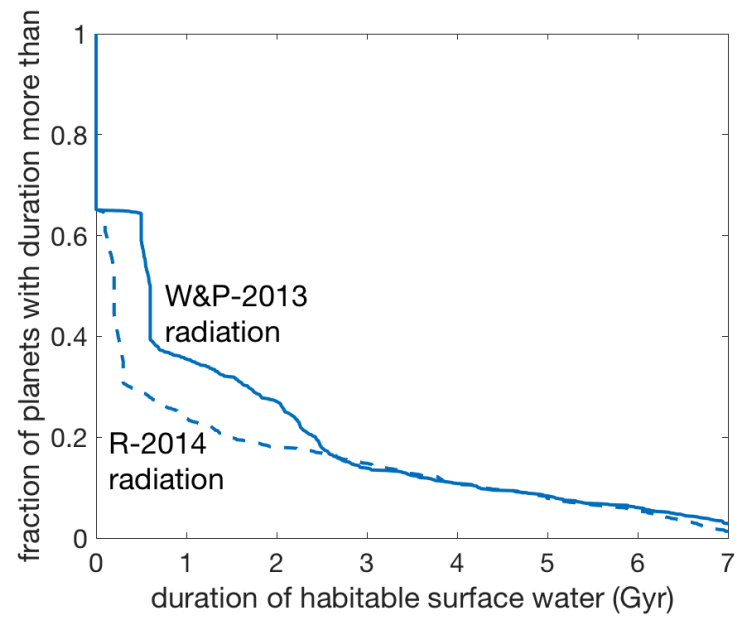

Figure 20. Comparison of the duration of habitable surface water obtained using the (Ramirez et al. 2014) climate model (dashed line, "R-2014 radiation"), versus the duration of habitable surface water obtained using the Wordsworth \& Pierrehumbert (2013a climate model (solid line, "W\&P-2013 radiation"). Planet semimajor axis $=1.1 \mathrm{AU}$, planet mass $=1$ Earth-mass, planet water mass fraction $f_{W}=0.01$.

habitable surface water for longer than the age of the Earth does not change (Figure 20).

Neither the model of Wordsworth \& Pierrehumbert (2013a) nor that of Ramirez et al. (2014) self-consistently models changes in the effect of clouds on planet albedo as $p_{\mathrm{CO} 2}$ increases. Neither model includes ice-albedo feedbacks. We can say little about the effect of planet mass because the gravity correction on the greenhouse effect is important, and not included in this study (Pierrehumbert 2010). On planets bigger than Earth, the highpermeability fractured zone in the uppermost crust is 
smaller (Sleep 2000b).

Ocean chemistry look-up grid. The ocean chemistry look-up grid was constructed using the Gibbs-freeenergy-minimization software CHIM-XPT (Reed 1998). We built an ocean chemistry look-up grid in the H-O$\mathrm{C}-\mathrm{Ca}-\mathrm{Na}$ system. We did this by varying the following component abundances in CHIM-XPT:- $\mathrm{Na}^{+}$(11 trials); $\mathrm{Ca}^{2+}$ (9 trials ); $\mathrm{HCO}_{3}^{-}$(12 trials). We also varied temperature (from 273.25 to $453.15 \mathrm{~K} ; 10$ trials). This gave a total of $11 \times 9 \times 12 \times 10=1.2 \times 10^{4}$ trials. Charge balance was enforced by setting the proton molality, $\left[\mathrm{H}^{+}\right]$, to $\left[\mathrm{HCO}_{3}^{-}\right]-2 \times\left[\mathrm{Ca}^{2+}\right]-\left[\mathrm{Na}^{+}\right]$. Here, the square brackets denote molality. We adjusted the specific values trialled for each of the four parameters, with the final values set non-uniformly in order to ensure good coverage of interesting behavior.

For convenience, we used CHIM-XPT in reactiontransport mode to solve the equilibration problem. Formally, we added a tiny amount of $\mathrm{H}_{2} \mathrm{O}$ to the system for a single reaction-transport step. CHIM-XPT polices charge balance using a "charge balance ion," which we shifted from $\mathrm{Na}$ to $\mathrm{Ca}$ as appropriate to avoid spurious crashes. We ran CHIM-XPT using the Windows emulator wine and shell scripts with CHIM-XPT settings minsolsv $=1$, ipsat $=0$, and $P=20$ bars. From the equilibrated output for each of the $1.2 \times 10^{4}$ trials , we grepped the abundances of the species $\mathrm{H}^{+}(\leftrightarrow \mathrm{pH})$; $\mathrm{HCO}_{3}^{-} ; \mathrm{CO}_{\mathrm{aq}} ; \mathrm{CO}_{3}^{-2} ; \mathrm{CaCO}_{3, \mathrm{aq}} ; \mathrm{Ca}\left(\mathrm{HCO}_{3}^{-}\right) ; \mathrm{CO}_{2(g)}$ (where present); calcite; dolomite; siderite; magnesite; and portlandite, to a summary text file.

This text file was ingested by script parse_ocean_surface_carbonate_chemistry.m. We interpolated to densify the grid to 180 temperatures $\left(1-180^{\circ} \mathrm{C}\right)$ and $\left.18 \mathrm{Na}^{+}\right]$cases. We then considered 21 cation-content cases: (1) $0.5 \mathrm{~mol} / \mathrm{kg} \mathrm{Na}$, minimal Ca; (2) cation-poor ("dilute") ocean; (3) minimal Na+, high $\mathrm{Ca}+$; (4) a sweep of 18 values of $\mathrm{Na}^{+}$concentration at minimal $\mathrm{Ca}$ concentration. The procedure of using equilibria at a fixed pressure (in this case 20 bar) unavoidably introduces errors at different sea-surface pressures - for example, Ca-bearing minerals are less stable at higher pressure. We also found that there were discrepancies between the saturation pressures interpolated from CHIM-XPT, and the values obtained from Carroll et al. (1991) and Duan \& Sun (2003), for the dilute-water case. We chose to use the published tables to get $\mathrm{CO}_{2}$ saturation pressures.

Ocean-atmosphere equilibriation. The ocean chemistry look-up table and the greenhouse forcing and climate look-up table are both passed to the climate evolution script, make_waterworld_fate_diagram.m. For waterworlds, this script takes inputs of planet mass $M_{p l}$, planet initial water mass fraction $f_{W}$, planet initial atmosphere+ocean carbon content $c_{C}$, and planet semimajor axis $a$. The output is ocean chemistry, atmospheric partial pressure of carbon dioxide $p_{\mathrm{CO} 2}$, and ocean surface temperature $T_{\text {surf }}$, as functions of time.

A necessary first step is to get the atmosphere-ocean partitioning of carbon. To do this, we use the tables of Duan \& Sun (2003) to get $\mathrm{CO}_{2, \text { aq }}$ as a function of $p_{\mathrm{CO} 2}$. We then use the CHIM-XPT output to get the $\mathrm{pH}-$ dependent "extra" $\mathrm{C}\left(\mathrm{HCO}_{3}^{-}, \mathrm{CO}_{3}^{2-}\right.$, and solid phases) stored in the ocean for a given $\mathrm{CO}_{2, \text { aq }}$. Multiplying the
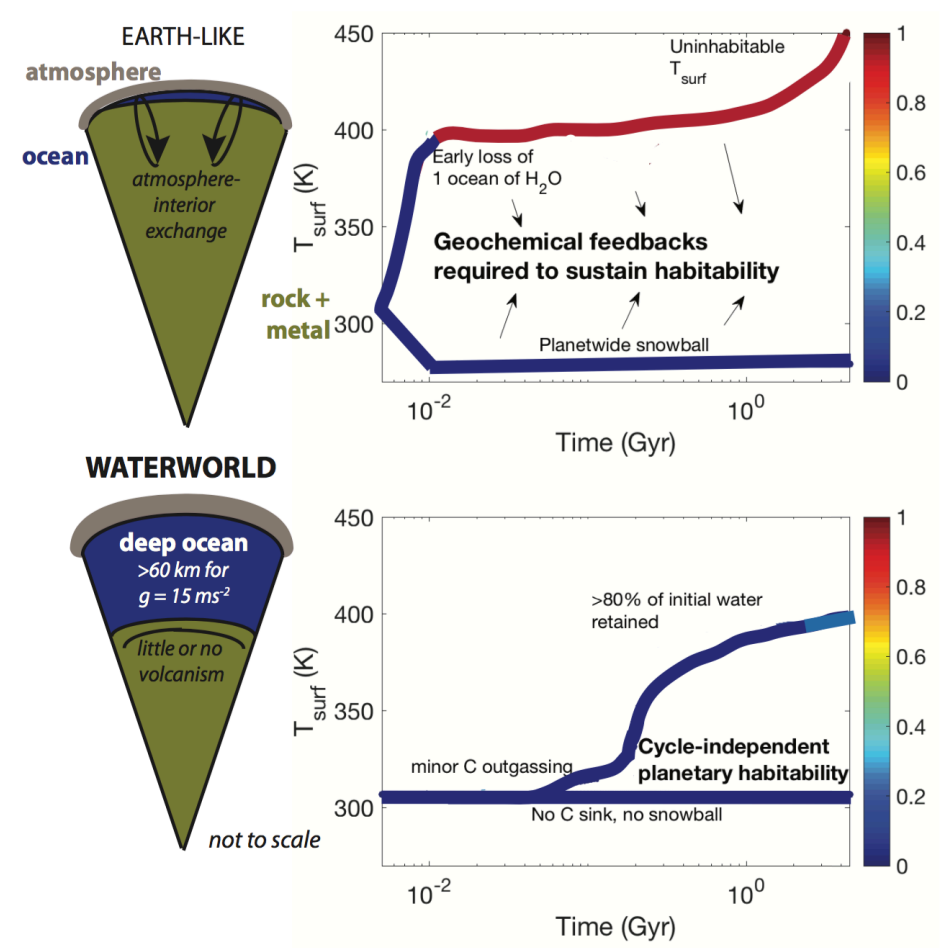

Figure 21. How the response of an Earth-mass planet to perturbations of the geologic $\mathrm{C}$ cycle differs depending on ocean depth (in a toy model). Upper panel: a planet with shallow oceans but no geochemical feedbacks. Lower panel: a waterworld. The two temperature vs. time tracks for each panel correspond to an increase (upper/hot track in each panel) and a decrease (lower/cold track in each panel) in the $\mathrm{CO}_{2}$ flux from the interior (crust+mantle) into the atmosphere+ocean. Color shows fraction of initial $\mathrm{H}_{2} \mathrm{O}$ lost to space, assuming XUV-energy-limited escape (Lammer et al. 2009) when and only when the stratospheric mixing ratio of water is large (Wordsworth \& Pierrehumbert 2013a). In the shallow-ocean case (upper panel), the climate rapidly becomes uninhabitable. Therefore, in this toy model, geochemical feedbacks are required to restore and sustain habitability. Specifically, suppose C supply by outgassing exceeds $\mathrm{C}$ uptake by weathering. Then, temperature will rise and trigger a moist greenhouse, which will dehydrate the world (hot track). On the other hand, cations made available for weathering by volcanism and tectonics can swiftly draw down atmospheric $\mathrm{CO}_{2}$ to negligible levels, triggering a snowball (cold track). Lower panel: in the deep-ocean case, the planet remains habitable for many Gyr due to the subdued geologic C-cycle, plus ocean dilution. Specifically, volcanism requires a much longer time to trigger the moist greenhouse and the planet stays habitable indefinitely in the moist greenhouse state, which cannot remove the massive initial ocean (hot track). On the other hand, without continents or volcanism, $\mathrm{C}$ drawdown quickly becomes cation-limited and diffusive, so the planet cannot enter the snowball state (cold track).

total ocean molality by the ocean column mass $\left(\mathrm{kg} / \mathrm{m}^{2}\right)$ gives the total moles of $\mathrm{C}$ in the ocean. Multiplying by $44 / 1000$ gives the $\mathrm{CO}_{2}$-equivalent $\mathrm{C}$ column in the ocean, and adding the atmospheric $\mathrm{CO}_{2}$ column mass $\left(\approx p_{\mathrm{CO} 2} / \mathrm{g}\right)$ gives the total $\mathrm{C}$ column $\left(\mathrm{kg} / \mathrm{m}^{2}\right)$ as a function of $p_{\mathrm{CO} 2}$. We then interpolate to find the $p_{\mathrm{CO} 2}$ for a given cation content and given total atmosphere+ocean C abundance.

\section{F. EXSOLUTION-DRIVEN CLIMATE INSTABILITIES.}

Here we provide more discussion of the modelpredicted instabilities mentioned in Section 4.1, some of which are shown in Figures 8 and 9

Increases in $p_{\mathrm{CO} 2}$ of up to a factor of 10 accompanied by increases in temperature of up to $100{ }^{\circ} \mathrm{C}$ can occur 
in the model within a single $(0.1 \mathrm{Gyr})$ timestep. How fast would warming occur? The pace of change is set by deep-ocean thermal inertia and by the ocean mixing time (which is $\sim 10^{3} \mathrm{yr}$ for Earth). If the ocean mixes slowly (i.e. is stratified), then the timescale for the instability could be as long as the conductive timescale, $>10^{7} \mathrm{yr}$. If instead the ocean mixing time is fast, then as each parcel of seawater is mixed into the near-surface layer with temperature $T_{\text {surf }}$, it will exsolve $\mathrm{CO}_{2}$. Therefore, both $p_{\mathrm{CO} 2}$ and $T_{\text {surf }}$ will rise on the fast ocean mixing timescale. Fast warming of the wave-mixed nearsurface layer $(<0.1 \mathrm{~km}$ depth) could stress phototrophs. However, for a $O(10) \mathrm{W} / \mathrm{m}^{2}$ change in the surface energy balance, a $100 \mathrm{~km}$-deep ocean will take $>100 \mathrm{Kyr}$ to warm. Can this rate of change exterminate life? Microbial populations can make major adaptations on 20yr timescales (Blount et al. 2008). Therefore the rate of change associated with these instabilities is unlikely to risk whole-ocean microbial extinction (in our opinion), provided that both the initial climate and final climate are habitable.

The existence of instabilities implies that surface energy balance for a given solar luminosity and given $\mathrm{CO}_{2}-$ equivalent atmosphere+ocean $\mathrm{C}$ content can be satisfied by more than one surface temperature. Which temperature is correct? The low-temperature solution (as long as it exists) is the one followed by our planet evolution tracks. This is because solar luminosity is initially low. Forced by $L_{*}$, whole-ocean $T$ will start low and increase with time, in the absence of other perturbations. One way to access the warm solutions is an asteroid impact. However the impacts would have to be very large, because of the large thermal inertia of the deep ocean. (A smaller impact can raise the temperature of the shallow ocean and exsolve some $\mathrm{CO}_{2}$, but accessing the stable warm branch requires exsolving $\mathrm{CO}_{2}$ from the bulk of the ocean.) For example, consider one of the largest impact structures on Mars, Hellas. If a third of Hellas' impact energy (total $\sim 4 \times 10^{27} \mathrm{~J}$; Williams \& Greeley $1994)$ goes into heating a $100 \mathrm{~km}$ deep ocean on a $1 M_{\oplus}$ planet, $\Delta T_{\text {surf }} \approx 5 \mathrm{~K}$, which is small. Nevertheless, impacts more energetic than Hellas are possible early in planetary history, and if the warm branch is accessed, then the trace of the impact will be seen in the climate for $O(\mathrm{Gyr})$. The time-integrated change in surface energy balance (units $\mathrm{J}$ ) as the consequence of the impact can easily be $1000 \times$ greater than the impact energy. This is a new mechanism for a metastable impact-driven climate. Metastable impact-triggered climates have previously been proposed using other mechanisms (Segura et al. 2012, Urata and Toon 2013, but see also Wordsworth 2016).

\section{REFERENCES}

Abbot, D. S., Cowan, N. B., \& Ciesla, F. J. 2012, ApJ, 756, 178 Abbot, D. S., Voigt, A., \& Koll, D. 2011, JGR-A, 116, D18103

Abe, Y., Ohtani, E., Okuchi, T., Righter, K., \& Drake, M. 2000, p.413- in Canup, R.M., \& K. Righter (Eds.) Origin of the Earth and Moon, University of Arizona Press.

Abe, Y., Abe-Ouchi, A., Sleep, N. H., \& Zahnle, K. J. 2011, Astrobiology, 11, 443

Abramson, E. H., \& Brown, J. M. 2004,

Geochim. Cosmochim. Acta, 68, 1827

Akanuma, S., Nakajima, Y., Yokobori, S.-i., et al. 2013, PNAS, 110,11067
Albarède, F. 2009, Nature, 461, 1227

Alibert, Y. 2014, A\&A, 561, A41

Alt, J. C. 1995, AGU Geophys. Monograph Series, 91, 85

Archer, D., Eby, M., Brovkin, V., et al. 2009, Ann. Rev. Earth Planet. Sci., 37, 117

Atreya, S. K., Lorenz, R. D., \& Waite, J. H. 2010, in Brown, R., Lebreton, J. L., \& Waite, H., (eds.), Titan from Cassini-Huygens, 177

Bahcall, J. N., Pinsonneault, M. H., \& Basu, S. 2001, ApJ, 555, 990

Bains, W., Xiao, Y., \& Yu, C., 2015, Life, 5(2), 1054-1100

Ballard, S., Chaplin, W. J., Charbonneau, D., et al. 2014, ApJ, 790, 12

Batygin, K., \& Laughlin, G. 2015, PNAS, 112, 4214

Beerling, D. 2008, The Emerald Planet, Oxford University Press

Bergin, E. A., Blake, G. A., Ciesla, F., et al., 2015, PNAS, 112, 8965

Blake, R. E., Chang, S. J., Lepland, A. 2010. Nature 464, 1029-1032.

Blättler, C. L., Kump, L. R., Fischer, W. W., et al. 2017, Nature Geoscience, 10, 41

Blount, Z. D., Borland, C. Z., \& Lenski, R. E. 2008, PNAS, 105, 7899

Bollengier, O., Choukroun, M., Grasset, O., et al. 2013,

Geochim. Cosmochim. Acta, 119, 322

Bourrier, V., de Wit, J., Bolmont, E., et al. 2017, AJ, 154, 121

Broecker, W. S., \& Sanyal, A. 1998, Global Biogeochemical Cycles, 12, 403

Bullock, M. A., \& Moore, J. M. 2007, Geophys. Res. Lett., 34, L19201

Burgasser, A. J., \& Mamajek, E. E. 2017, ApJ, 845, 110

Burger, C., Maindl, T. I., \& Schäfer, C. M. 2018, Celestial

Mechanics and Dynamical Astronomy 130, \#2.

Burke, C. J., Christiansen, J. L., Mullally, F., et al. 2015, ApJ, 809,8

Butler, J. N., Carbon dioxide equilibria and their applications, Addison-Wesley

Caldeira, K., \& Kasting, J. F. 1992, Nature, 359, 226

Cannon, K. M., Parman, S. W., \& Mustard, J. F. 2017, Nature

Carroll, J. J., Slupsky, J. D., \& Mather, A. E. 1991, Journal of Physical and Chemical Reference Data, 20, 1201

Carrozzo, F.G., et al., 2018, Science Advances, 4, doi:10.1126/sciadv.1701645.

Carter, B. 1983, RSPTA, 310, 347

Catling, D. C., \& Kasting, J. F. 2017, Atmospheric Evolution on Inhabited and Lifeless Worlds, Cambridge University Press

Chachan, Y., Stevenson, D. J. 2018. ApJ854, 21.

Ciesla, F. J., Mulders, G. D., Pascucci, I., \& Apai, D. 2015, ApJ, 804,9

Clay, P. L., Burgess, R., Busemann, H., et al. 2017, Nature, 551, 614

Coogan, L. A., Parrish, R. R., \& Roberts, N. M. W. 2016 , Geology, 44, 147

Coogan, L. A., Gillis, K. M. 2018. Low-Temperature Alteration of the Seafloor: Impacts on Ocean Chemistry. Annual Review of Earth and Planetary Sciences 46, 21-45.

COEL (Committee on the Origins and Evolution of Life), 2007, The Limits of Organic Life in Planetary Systems, National Research Council, ISBN: 0-309-66906-5, 116 pp.

Cossou, C., Raymond, S. N., Hersant, F., et al., 2014, A\&A, 569, A56

Cowan, N. B., Abbot, D. S. 2014. Water Cycling between Ocean and Mantle: Super-Earths Need Not Be Waterworlds. ApJ781, 27.

Dahl, T. W., \& Stevenson, D. J. 2010, E\&PSL , 295, 177

Dasgupta, R. 2013, Rev. Mineral. Geochem., 75, 183

Dolejš, D., \& Manning, C. E. 2010, Geofluids, 10, 20-40

Dong, C., Huang, Z., Lingam, M., et al. 2017, ApJ, 847, L4

Dong, C., Jin, M., Lingam, M., Airapetian, V. S., Ma, Y., van der Holst, B. 2018. Proceedings of the National Academy of Science $115,260-265$.

Dorn, C., Khan, A., Heng, K., et al. 2015, A\&A, 577, A83

Dorn, C., Bower, D. J., Rozel, A. 2017. Assessing the Interior Structure of Terrestrial Exoplanets with Implications for Habitability. Handbook of Exoplanets, Edited by Hans J. Deeg and Juan Antonio Belmonte. Springer Living Reference Work, ISBN: 978-3-319-30648-3, 2017, id.66 66. 
Dorn, C., Noack, L., Rozel, A. 2018. Astronomy and Astrophysics 614, A18.

Dressing, C. D., \& Charbonneau, D. 2015, ApJ, 807, 45

Duan, Z., \& Sun, R. 2003, Chemical Geology, 193, 257

Dwardakas, V.V., Dauphas, N., Meyer, B., Boyajian, P., \& Bojazi, M. 2003, ApJ, 851:147

Edmond, J. M., \& Huh, Y. 2003, E\&PSL , 216, 125

Edwards, C. S., \& Ehlmann, B. L. 2015, Geology, 43, 863

Elkins-Tanton, L. T. 2011. Formation of early water oceans on rocky planets. Astrophysics and Space Science 332, 359-364.

Fegley, B., Jr., Jacobson, N. S., Williams, K. B., et al. 2016, ApJ, 824, 103

Fisher, A. T. 1998, Rev. Geophys., 36, 143

Flament, N., Coltice, N., \& Rey, P.F., 2016, Earth and Planetary Science Letters, 275, 326-336.

Foley, B. J. 2015, ApJ, 812, 36

Foley, B. J., \& Smye, A. J. 2018, Astrobiology, https://doi.org/10.1089/ast.2017.1695

Ford, E. B., \& Rasio, F. A. 2008, ApJ, 686, 621-636

Friedson, A. J., \& Gonzales, E. J. 2017, Icarus, 297, 160

Friend, C. R. L., Nutman, A. P., Bennett, V. C., Norman, M. D. 2008. Contributions to Mineralogy and Petrology 155, 229-246.

Frost, B. R., Beard, J. S. 2007. Journal of Petrology 48, 1351-1368.

Fu, R., O'Connell, R. J., \& Sasselov, D. D. 2010, ApJ, 708, 1326

Gaidos, E., Deschenes, B., Dundon, L., et al. 2005, Astrobiology, 5,100

Gaidos, E., Krot, A. N., Williams, J. P., \& Raymond, S. N. 2009, ApJ, 696, 1854

Galvez, M. E., Connolly, J. A. D., \& Manning, C. E. 2016, Nature, 539, 420

Galbraith, E. D., \& Eggleston, S. 2017, Nature Geoscience, 10, 295

Gale, A., Dalton, C. A., Langmuir, C. H., Su, Y., \& Schilling, J.-G. 2013, Geochemistry, Geophysics, Geosystems, 14, 489

Galy, V., Peucker-Ehrenbrink, B., \& Eglinton, T. 2015, Nature, 521, 204

Garcia, A. K., Schopf, J. W., Yokobori, S.-i., Akanuma, S., Yamagishi, A. 2017. Proceedings of the National Academy of Science 114, 4619-4624.

Genda, H., \& Abe, Y. 2005, Nature, 433, 842

Genda, H., 2016, Geochemical Journal 50, 27-42.

Genda, H., Iizuka, T., Sasaki, T., Ueno, Y., Ikoma, M. 2017. Earth and Planetary Science Letters 470, 87-95.

Gillon, M., Triaud, A. H. M. J., Demory, B.-O., et al. 2017, Nature, 542, 456

Glein, C. R., Baross, J. A., \& Waite, J. H. 2015 Geochim. Cosmochim. Acta, 162, 202

Golabek, G. J., Emsenhuber, A., Jutzi, M., Asphaug, E. I., Gerya, T. V. 2018. Icarus 301, 235-246.

Goldblatt, C., Robinson, T. D., Zahnle, K. J., \& Crisp, D. 2013, Nature Geoscience, 6, 661

Goldblatt, C. 2015, Astrobiology, 15, 362

Gounelle, M. 2015, A\&A, 582, A26

Grimm, S. L., and 25 colleagues 2018. The nature of the TRAPPIST-1 exoplanets. Astronomy and Astrophysics 613, A68.

Grotzinger, J. P., \& Kasting, J. F. 1993, J. Geol., 101, 235

Gysi, A. P., \& Stefánsson, A. 2012, Chemical Geology, 306, 10

Halevy, I., \& Schrag, D. P. 2009, Geophys. Res. Lett., 36, L23201

Halevy, I., \& Bachan, A. 2017, Science, 355, 1069

Hayes, J.M., \& Waldbauer, J.R. 2006, Phil. Trans. Roy. Soc. B, 361(1470), 931-50

Hamano, K., Abe, Y., \& Genda, H. 2013, Nature, 497, 607

Hand, K. P., \& Chyba, C. F. 2007, Icarus, 189, 424

Hannington, M. D., de Ronde, C. E. J., \& Peterson, S. 2005, Economic Geology, 100th Anniversary Volume, 111-141

Hartmann, L., Ciesla, F., Gressel, O., \& Alexander, R. 2017, Space Science Reviews 212, 813-834.

Henning, W. G., O'Connell, R. J., Sasselov, D. D. 2009. Tidally Heated Terrestrial Exoplanets: Viscoelastic Response Models. ApJ707, 1000-1015.

Herzberg, C., Condie, K., \& Korenaga, J. 2010, E\&PSL , 292, 79

Hoffman, P.F., Abbot, D.S., Ashkenazy, Y., et al. 2017, Science Advances, 3, DOI: 10.1126/sciadv.1600983.

Holland, H. D. 1984, The chemical evolution of the atmosphere and oceans. Princeton University Press, Princeton, N.J., USA.
Hirschmann, M. M. 2006, Ann. Rev. Earth Planet. Sci., 34, 629 Hirschmann, M. M. 2012, E\&PSL, 341, 48

Hirschmann, M., \& Kohlstedt, D. 2012, Physics Today, 65, 40 Hirschmann, M. M. 2016, American Mineralogist, 101, 540

Hsu, H.-W., Postberg, F., Sekine, Y., et al. 2015, Nature, 519, 207

Hu, Y., Yang, J., Ding, F., et al., 2011, Clim. Past, 7, 17

The International Association for the Properties of Water and Steam, 2011, Revised Release on the Pressure along the Melting and Sublimation Curves of Ordinary Water Substance, IAPWS R14-08(2011).

Inamdar, N. K., \& Schlichting, H. E. 2016, ApJ, 817, L13

Ingersoll, A. P. 1969. The Runaway Greenhouse: A History of Water on Venus.. Journal of Atmospheric Sciences 26, 1191-1198.

Jacobsen, S. B., Ranen, M. C., Petaev, M. I., et al. 2008, RSPTA, 366,4129

Jacobson, S. A., Rubie, D. C., Hernlund, J., Morbidelli, A., \& Nakajima, M. 2017, E\&PSL , 474, 375

Jura, M., Xu, S., \& Young, E. D. 2013, ApJ, 775, L41

Izidoro, A., Ogihara, M., Raymond, S. N., et al. 2017, MNRAS, 470,1750

Kah, L. C., \& Riding, R. 2007, Geology, 35, 799

Kalousová, K., Sotin, C., Choblet, G., Tobie, G., \& Grasset, O. 2018, Icarus, 299, 133

Kalousová, K., Sotin, C., Geophys. Res. Lett. https://doi.org/10.1029/2018GL078889

Kaltenegger, L. 2017, ARA\&A, 55, 433

Kasting, J. F., Ackerman, T. P. 1986. Science 234, 1383-1385.

Kasting, J. F. 1988, Icarus, 74, 472

Kasting, J. F., Holm, N. G. 1992. What determines the volume of the oceans?. Earth and Planetary Science Letters 109, 507-515.

Kasting, J. F., Whitmire, D. P., \& Reynolds, R. T. 1993, Icarus, 101,108

Kasting, J. F., \& Catling, D. 2003, ARA\&A, 41, 429

Kasting, J. F., Kopparapu, R., Ramirez, R. M., \& Harman, C. E. 2014, PNAS, 111, 12641

Katz, R. F., Spiegelman, M., \& Langmuir, C. H. 2003, Geochemistry, Geophysics, Geosystems, 4, 1073

Kelemen, P. B., \& Hirth, G. 2012, E\&PSL, 345, 81

Kelemen, P. B., \& Manning, C. E. 2015, PNAS, 112, E3997

Kempe, S., \& Degens, E. T. 1985, Chemical Geology, 53, 95

Kite, E. S., Manga, M., \& Gaidos, E. 2009, ApJ, 700, 1732

Kite, E. S., Gaidos, E., \& Manga, M. 2011, ApJ, 743, 41

Kitzmann, D., Alibert, Y., Godolt, M., et al. 2015, MNRAS, 452, 3752

Klein, F., Bach, W., McCollom, T. M. 2013. Lithos 178, 55-69.

Knoll, A. H., Canfield, D. E., \& Konhauser, K. O. (editors) 2012 Fundamentals of Geobiology, Wiley-Blackwell, 2012,

Kodama, T., Genda, H., Abe, Y., \& Zahnle, K. J. 2015, ApJ, 812, 165

Kodama, T., Nitta, A., Genda, H., Takao, Y., O'ishi, R., Abe-Ouchi, A., Abe, Y. 2018. JGR (Planets) doi:10.1002/2017JE005383.

Komacek, T. D., \& Abbot, D. S. 2016, ApJ, 832, 54

Kopp, R. E., Kirschvink, J. L., Hilburn, I. A., \& Nash, C. Z. 2005, PNAS, 102, 11131

Korenaga, J., \& Karato, S.-I. 2008, JGR (Solid Earth), 113, B02403

Korenaga, J. 2013, Ann. Rev. Earth and Planet. Sci., 41, 117

Korenaga, J., Planavsky, N. J., \& Evans, D. A. D. 2017, Phil. Trans. Roy. Soc. A, 375, 20150393

Kopparapu, R. K., Ramirez, R., Kasting, J. F., et al. 2013, ApJ, 765,131

Kopparapu, R. K., Ramirez, R. M., SchottelKotte, J., et al. 2014, ApJ, 787, L29

Kopparapu, R. k., Wolf, E. T., Arney, G., et al. 2017, ApJ, 845, 5

Krissansen-Totton, J., \& Catling, D. C. 2017, Nature

Communications, 8, 15423

Krissansen-Totton, J., Arney, G. N., Catling, D. C. 2018.

Proceedings of the National Academy of Science 115, 4105-4110.

Kuchner, M. J. 2003, ApJ, 596, L105

Kulikov, Y. N., and 10 colleagues 2006. Planetary and Space Science 54, 1425-1444.

Kump, L. R., Brantley, S. L., Arthur, M. A. 2000. Chemical Weathering, Atmospheric $\mathrm{CO}_{2}$, and Climate. Annual Review of Earth and Planetary Sciences 28, 611-667. 
Kuramoto, K., \& Matsui, T. 1996, J. Geophys. Res., 101, 14909 Lacki, B. C. 2016, arXiv:1609.05931

Lammer, H., Odert, P., Leitzinger, M., et al. 2009, A\&A, 506, 399

Leconte, J., Forget, F., Charnay, B., Wordsworth, R., \& Pottier, A. 2013, Nature, 504, 268

Léger, A., Selsis, F., Sotin, C., et al. 2004, Icarus, 169, 499

Levi, A., Sasselov, D., \& Podolak, M. 2014, ApJ, 792, 125

Levi, A., Sasselov, D., \& Podolak, M. 2017, ApJ, 838, 24

Levi, A., Sasselov, D. 2018. The Astrophysical Journal 857, 65.

Levison, H. F., Kretke, K. A., Walsh, K. J., et al. 2015, PNAS, 112,14180

Lichtenberg, T., Parker, R. J., \& Meyer, M. R. 2016, MNRAS, 462,3979

Loeb, A., Batista, R. A., \& Sloan, D. 2016, JCAP, 8, 040

Löhne, T., Krivov, A. V., \& Rodmann, J. 2008, ApJ, 673, 1123-1137

Luger, R., Barnes, R. 2015. Astrobiology 15, 119-143.

Luger, R., Sestovic, M., Kruse, E., et al. 2017, Nature Astronomy, 1,0129

Lupu, R. E., Zahnle, K., Marley, M. S., et al. 2014, ApJ, 784, 27

MacLeod, G., McKeown, C., Hall, A. J., \& Russell, M. J. 1994, Origins of Life and Evolution of the Biosphere, 24, 19

Maher, K., \& Chamberlain, C. P. 2014, Science, 343, 1502

Maindl, T. I., Schäfer, C. M., Haghighipour, N., Burger, C., \& Dvorak, R. 2017, Proc. of the 1st Greek-Austrian Workshop on Extrasolar Planetary Systems, 137

Marcus, R. A., Sasselov, D., Stewart, S. T., \& Hernquist, L. 2010a, ApJ, 719, L45

Marcus, R. A., Sasselov, D., Hernquist, L., \& Stewart, S. T. 2010b, ApJ, 712, L73

Marinova, M. M., Aharonson, O., Asphaug, E. 2008. Nature 453, 1216-1219.

Marty, B. 2012, Earth and Planetary Science Letters, 313, 56

Marty, B., Avice, G., Sano, Y., Altwegg, K., Balsiger, H., Hässig, M., Morbidelli, A., Mousis, O., Rubin, M. 2016.. Earth and Planetary Science Letters, 441, 91

McKinnon, W. B., \& Zolensky, M. E. 2003, Astrobiology, 3, 879

Melosh, J. 2011, Planetary Surface Processes, Cambridge University Press.

Mezger, K., Debaille, V., \& Kleine, T. 2013, Space Sci. Rev., 174 27

Marounina, N., \& Rogers, L.A., 2017, poster at The Origins of Volatiles in Habitable Planets, workshop held in Ann Arbor, MI

Marounina, N., Grasset, O., Tobie, G., \& Carpy, S. 2018, Icarus 310, 127-139

Moll, R., Garaud, P., Mankovich, C., \& Fortney, J. J. 2017, ApJ, 849,24

Monteux, J., Golabek, G. J., Rubie, D. C., Tobie, G., \& Young, E. D. 2018, Space Sci. Rev., 214, \#39

Moore, G., Vennemann, T., \& Carmichael, I. S. E. 1998, American Mineralogist, 83, 36

Moore, W. B., Lenardic, A., Jellinek, A. M., et al. 2017, Nature Astronomy, 1, 0043

Morbidelli, A., Bitsch, B., Crida, A., et al. 2016, Icarus, 267, 368

Moynier, F., Yin, Q.-Z., Irisawa, K., et al. 2010, PNAS, 107, 10810

Mulders, G. D., Ciesla, F. J., Min, M., \& Pascucci, I. 2015, ApJ, 807,9

Mustard, J.F., 2018, Sequestration of volatiles in the Martian crust through hydrated minerals: a significance planetary reservoir of water, chapter 8 in Volatiles in the Martian crust, edited by J. Filiberto and S. Schwenzer, Elsevier.

Myint, P. C., Benedict, L. X., \& Belof, J. L. 2017 J. Chem. Phys., 147, 084505

Nakamura, K., \& Kato, Y. 2004, Geochim. Cosmochim. Acta, 68, 4595

Neveu, M., Desch, S. J., \& Castillo-Rogez, J. C. 2015, JGR (Planets), 120, 123

Nimmo, F., Hart, S. D., Korycansky, D. G., Agnor, C. B. 2008. Nature 453, 1220-1223.

Nitschke, W., McGlynn, S. E., Milner-White, E. J., \& Russell, M. J. 2013, Biochim Biophys Acta 1827(8-9):871-81

Noack, L., Breuer, D. 2014. Planetary and Space Science 98, 41-49.

Noack, L., Höning, D., Rivoldini, A., et al. 2016, Icarus, 277, 215

Noack, L., Rivoldini, A., Van Hoolst, T. 2017. Physics of the Earth and Planetary Interiors 269, 40-57.
O'Brien, D. P., Walsh, K. J., Morbidelli, A., Raymond, S. N., \& Mandell, A. M. 2014, Icarus, 239, 74

O’Brien, D. P., Izidoro, A., Jacobson, S. A., Raymond, S. N., \& Rubie, D. C. 2018, Space Sci. Rev., 214, 47

Odert, P., Lammer, H., Erkaev, N. V., et al. 2018, Icarus 307, 327-346.

Ogihara, M., Morbidelli, A., \& Guillot, T. 2015, A\&A, 578, A36

Ohtani, E. 2005, Elements 1(1), 25-30.

O'Rourke, J. G., \& Korenaga, J. 2012, Icarus, 221, 1043

Owen, J. E., \& Alvarez, M. A. 2016, ApJ, 816, 34

Owen, J. E., Mohanty, S. 2016. MNRAS459, 4088-4108.

Palme, H., \& O'Neill, H.St.C. 2014. Cosmochemical Estimates of Mantle Composition, chapter 3.1 in Treatise on Geochemistry 2nd Edition dx.doi.org/10.1016/B978-0-08-095975-7.00201-1.

Pan, D., Spanu, L., Harrison, B., Sverjensky, D. A., \& Galli, G. 2013, PNAS, 110, 6646

Pierrehumbert, R. T. 2010, Principles of Planetary Climate, Cambridge University Press.

Piso, A.-M. A., Öberg, K. I., Birnstiel, T., \& Murray-Clay, R. A. 2015, ApJ, 815, 109

Plesa, A.-C., Tosi, N., \& Breuer, D. 2014, E\&PSL, 403, 225

Pollack, J. B., Kasting, J. F., Richardson, S. M., Poliakoff, K. 1987. Icarus 71, 203-224.

Popp, M., Schmidt, H., \& Marotzke, J. 2016, Nature Communications, 7, 10627

Radko, T. 2013, Double Diffusive Convection, Cambridge University Press.

Ramirez, R. M., Kopparapu, R. K., Lindner, V., Kasting, J. F. 2014. Astrobiology 14, 714-731.

Ramirez, R. M., Kaltenegger, L. 2014. ApJ, 797, L25.

Ramirez, R. M., \& Kaltenegger, L. 2017, ApJ, 837, L4

Ramirez, R. M., Levi, A. 2018. Monthly Notices of the Royal Astronomical Society 477, 4627-4640.

Raymond, S. N., Quinn, T., \& Lunine, J. I. 2004, Icarus, 168, 1

Raymond, S. N., Quinn, T., \& Lunine, J. I. 2007, Astrobiology, 7, 66

Raymond, S. N., Boulet, T., Izidoro, A., Esteves, L., Bitsch, B. 2018. MNRAS479, L81-L85.

Redfern, S. A. T., Salje, E., \& Navrotsky, A. 1989, Contributions to Mineralogy and Petrology, 101, 479

Redmer, R., Mattsson, T. R., Nettelmann, N., \& French, M. 2011, Icarus, 211, 798

Reed, M. H. 1998, pp. 109-124 in Techniques in Hydrothermal Ore Deposits Geology, edited by J. P. Richards \& P. B. Larson, Society of Economic Geologists, Littleton, CO.

Rein, H. \& Liu, S.-F. 2012, å, 537, 128

Rein, H. \& Spiegel, D.S. 2015, MNRAS, 446, 1424

Ridgwell, A., \& Zeebe, R. E. 2005, E\&PSL , 234, 299

Rogers, L. A., \& Seager, S. 2010, ApJ, 712, 974

Rogers, L. A., chapter 5 in Phd Thesis, MIT, http://hdl.handle.net/1721.1/77254

Rogers, L. A. 2015. Most 1.6 Earth-radius Planets are Not Rocky. ApJ801, 41.

Ronco, M. P., de Elía, G. C. 2018. Formation of Solar system analogues II: post-gas phase growth and water accretion in extended discs via N-body simulations. Monthly Notices of the Royal Astronomical Society

Rosenbauer, R. J., Bischoff, J. L., \& Zierenberg, R. A. 1988, Journal of Geology, 96, 237

Rosenberg, N. D., Browning, L., \& Bourcier, W. L. 2001, M\&PS, 36, 239

Rothman, D. H., \& Forney, D. C. 2007, Science, 316, 1325

Rubie, D. C., Jacobson, S. A., Morbidelli, A., et al. 2015, Icarus, 248,89

Rubin, K. H., van der Zander, I., Smith, M. C., \& Bergmanis, E. C. 2005 , Nature, 437,534

Russell, M. J., \& Arndt, N. T. 2005, Biogeosciences, 2, 97

Russell, J. F., Hall, A. J., \& Martin, W. 2010, Geobiology, 8, 355

Sato, T., Okuzumi, S., \& Ida, S. 2016, A\&A, 589, A15

Schaefer, L., Lodders, K., Fegley, B. 2012. ApJ, 755, 41.

Schaefer, L., Sasselov, D. 2015. ApJ, 801, 40.

Schaefer, L., Wordsworth, R. D., Berta-Thompson, Z., \&

Sasselov, D. 2016, ApJ, 829, 63

Schlichting, H. E., Sari, R., \& Yalinewich, A. 2015, Icarus, 247, 81 
Schönbächler, M., Carlson, R. W., Horan, M. F., Mock, T. D., Hauri, E. H. 2010. Heterogeneous Accretion and the Moderately Volatile Element Budget of Earth. Science 328, 884 Schoonen, M., \& Smirnov, A. 2016, Elements, 12, 395-400

Schrenk, M. O., Kelley, D. S., Delaney, J. R., \& Baross, J.A. 2003, Applied \& Environmental Microbiology 69, 3580-3592.

Schubert, G., Turcotte, D. L., Olson, P. 2001. Mantle Convection in the Earth and Planets. pp. 956. ISBN 052135367X. Cambridge, UK: Cambridge University Press, September 2001. 956.

Segura, T. L., McKay, C. P., \& Toon, O. B. 2012, Icarus, 220, 144

Selsis, F., Chazelas, B., Bordé, P., et al. 2007, Icarus, 191, 453

Seyfried, W. E., Jr. 1987, Ann. Rev. Earth Planet. Sci., 15, 317

Sharma, A., Scott, J. H., Cody, G. D., et al. 2002, Science, 295, 1514

Sharp, Z. D., \& Draper, D. S. 2013, E\&PSL , 369, 71

Shibuya, T., Tahata, M., Kitajima, K., et al. 2012, E\&PSL , 321, 64

Shibuya, T., Yoshizaki, M., Masaki, Y., et al. 2013, Chemical Geology, 359, 1

Silburt, A., Gaidos, E., \& Wu, Y. 2015, ApJ, 799, 180

Sillen, L. G. 1967, Science, 156, 1189

Simpson, F. 2017, MNRAS, 468, 2803

Sleep, N. H. 2000, J. Geophys. Res., 105, 17563

Sleep, N. H. 2000, Geologic processes and habitability, in Proceedings of the Pale Blue Dot 2 Workshop, May 19-21, 1999, Ames Research Center

Sleep, N. H., Zahnle, K., \& Neuhoff, P. S. 2001, PNAS, 98, 3666

Sleep, N. H., \& Zahnle, K. 2001, J. Geophys. Res., 106, 1373

Sleep, N. H., Meibom, A., Fridriksson, T., Coleman, R. G., \& Bird, D. K. 2004, PNAS, 101, 12818

Sleep, N. H., Zahnle, K. J., \& Lupu, R. E. 2014, RSPTA, 372, 20130172

Solomatov, V. S. 2015, in Treatise on Geophysics (2nd. Edn).

Stamenković, V., \& Seager, S. 2016, ApJ, 825, 78

Stevenson, D. J. 1999, Nature, 400, 32

Stevenson, D. J. 2003, Comptes Rendus Geoscience, 335, 99

Stewart, S. T., Lock, S. J., \& Mukhopadhyay, S. 2014, Lunar and Planetary Science Conference, 45, 2869

Staudigel, H., \& Hart, S. R. 1983, Geochim. Cosmochim. Acta, 47, 337

Stolper, D. A., Bender, M. L., Dreyfus, G. B., Yan, Y., \& Higgins, J. A. 2016, Science, 353, 1427

Sun, Z., Ji, J., Wang, S., \& Jin, S. 2017, MNRAS, 467, 619

Takai, K., Nakamura, K., Toki, T., et al. 2008, PNAS, 105, 10949

Tartèse, R., Chaussidon, M., Gurenko, A., et al. 2017, Geochemical Perspective Letters, 3, n1.

Taylor, S. R., \& McLennan, S. 2009, Planetary Crusts. Cambridge University Press

Thomas, P. C., Parker, J. W., McFadden, L. A., et al. 2005, Nature, 437, 224

Tian, F. 2015, E\&PSL , 432, 126

Tian, F., \& Ida, S. 2015, Nature Geoscience, 8, 177

Tian, B.Y., \& Stanley, S. 2015, ApJ, 768:156

Tikoo, S. M., \& Elkins-Tanton, L. T. 2017, RSPTA, 375, 20150394

Tosi, N., Godolt, M., Stracke, B., et al. 2017, A\&A, 605, A71

Turbet, M., Forget, F., Leconte, J., Charnay, B., \& Tobie, G. 2017, E\&PSL, 476, 11

Turbet, M., Bolmont, E., Leconte, J., et al. 2018, Astronomy and Astrophysics 612 , A86.

Turcotte, D. L., \& Schubert, G. 2011, Geodynamics - 3rd Edition, Cambridge University Press.

Turner, S., Evans, P., \& Hawkesworth, C. 2001, Science, 292, 1363

Unterborn, C. T., Desch, S. J., Hinkel, N., \& Lorenzo, A. 2018, Nature Astronomy.

Urata, R. A., Toon, O. B. 2013. Icarus 226, 229-250.

Valencia, D., Sasselov, D. D., \& O'Connell, R. J. 2007, ApJ, 665, 1413
Valencia, D., Tan, V. Y. Y., Zajac, Z. 2018. The Astrophysical Journal 857, 106

Vance, S., Harnmeijer, J., Kimura, J., et al. 2007, Astrobiology, 7, 987

Vance, S., \& Brown, J. M. 2013, Geochim. Cosmochim. Acta, 110,176

Vance, S. D., Hand, K. P., \& Pappalardo, R. T. 2016, Geophys. Res. Lett., 43, 4871

Van Eylen, V., Agentoft, C., Lundkvist, M. S., Kjeldsen, H., Owen, J. E., Fulton, B. J., Petigura, E., Snellen, I. 2018. Monthly Notices of the Royal Astronomical Society 479, 4786-4795.

Vermeij, G. J. 2006, PNAS, 103, 1804

Walker, J. C. G., Hays, P. B., \& Kasting, J. F. 1981, J. Geophys. Res., 86, 9776

Ward, B., p. 36-48 in Fundamentals of Geobiology, First Edition, 2012, Edited by Andrew H. Knoll, Donald E. Canfield and Kurt O. Konhauser, Blackwell Publishing Ltd.

White, A. F., \& Brantley, S. L. 1995, Rev. Mineral. Geochem., 31.

White, S. M., Crisp, J. A., \& Spera, F. J. 2006, Geochemistry, Geophysics, Geosystems, 7, Q03010

White, W.M., \& Klein, E.M. 2014, Composition of the oceanic crust, chapter 4.13 in Treatise on Geochemistry (2nd Edition), H.D. Holland \& K.K. Turekian, Eds, Elsevier, Oxford.

Williams, D. A., \& Greeley, R. 1994, Icarus, 110, 196

Wiryana, S., Slutsky, L. J., \& Brown, J. M. 1998, E\&PSL, 163, 123

Wolf, E. T., \& Toon, O. B. 2015, JGR (Atmospheres), 120, 5775

Wolf, E. T., Shields, A. L., Kopparapu, R. K., Haqq-Misra, J., \&

Toon, O. B. 2017, ApJ, 837, 107

Wordsworth, R. 2012, Icarus, 219, 267

Wordsworth, R. D., \& Pierrehumbert, R. T. 2013, ApJ, 778, 154

Wordsworth, R., Pierrehumbert, R. 2013. Hydrogen-Nitrogen Greenhouse Warming in Earth's Early Atmosphere. Science 339,64 .

Wordsworth, R. D. 2016, Ann. Rev. Earth Planet. Sci., 44, 381

Wordsworth, R., Schaefer, L., \& Fischer, R. 2018, The Astronomical Journal 155, 195.

Workman, R. K., Hart, S. R. 2005. Major and trace element composition of the depleted MORB mantle (DMM). Earth and Planetary Science Letters 231, 53-72.

Yang, J., Leconte, J., Wolf, E. T., et al. 2016, ApJ, 826, 222

Yang, J., Ding, F., Ramirez, R. M., et al. 2017, Nature Geoscience, 10, 556

Young, E. D. 2001, RSPTA, 359, 2095

Zahnle, K. J., Kasting, J. F., \& Pollack, J. B. 1988, Icarus, 74, 62

Zahnle, K., Arndt, N., Cockell, C., et al. 2007, Space Sci. Rev., 129,35

Zahnle, K. J., Lupu, R., Dobrovolskis, A., \& Sleep, N. H. 2015, E\&PSL, 427,

Zahnle, K. J., \& Catling, D. C. 2017, ApJ, 843, 122

Zain, P. S., de Elía, G. C., Ronco, M. P., Guilera, O. M. 2018. Planetary formation and water delivery in the habitable zone around solar-type stars in different dynamical environments. Astronomy and Astrophysics 609, A76.

Zeebe, R. E. 2012, Ann. Rev. Earth Planet. Sci., 40, 141

Zeebe, R. E., \& Wolf-Gladrow, D. 2001, CO2 in Seawater:

Equilibrium, Kinetics, Isotopes, vol. 65, Elsevier Oceanography Series

Zeebe, R. E., \& Caldeira, K. 2008, Nature Geoscience, 1, 312

Zhou, J.-L., \& Lin, D. N. C. 2007, ApJ, 666, 447

Zolotov, M. Y. 2007, Geophys. Res. Lett., 34, L23203

Zolotov, M. Y., \& Kargel, J. S. 2009, p.431-457 in Europa, edited by R.T. Pappalardo, W.B. McKinnon, K.K. Khurana; University of Arizona Press. 